\title{
Efficient enantioselective synthesis of condensed and aromatic ring-substituted tyrosine derivatives
}

\author{
Sebastian Knör, Burkhardt Laufer and Horst Kessler* \\ Department Chemie, Lehrstuhl II für Organische Chemie, Technische Universität München, \\ Lichtenbergstr. 4, D-85747 Garching, Germany
}

\section{SUPPORTING INFORMATION}

General.

Synthesis of $(S)-N^{\alpha}$-tert-Butyloxycarbonyl-m-iodotyrosine (5).

S2

Synthesis of 3-aryl-substituted tyrosine analogues $\mathbf{7 b}$-j. General procedure.

Synthesis of $\mathbf{8 b}$ and $\mathbf{9 b}$.

Synthesis of 4-(tert-Butyldimethylsilyloxy)naphthalene-1-carbaldehyde (12).

S7

Solid-phase synthesis of dipeptides 10a-d, 16 and 17. General procedure.

NMR analyses of 4-6, 7a-j, 8a, 8b, 9a, 9b, 11-22.

S10-S37

NMR and HPLC analyses of dipeptides 10a-d, 16 and 17.

S38-S46

References.

S47

*Corresponding author: Tel: +49-089-289 13300, Fax: +49-089-289 13210, e-mail: Horst.Kessler@ch.tum.de 
General. All commercially available chemical reagents were used without further purification. Technical solvents were distilled before use. Thin-layer chromatography (TLC) was performed on aluminium-backed plates Merck silica gel $60 \mathrm{~F}_{254}$. Compounds were visualized by UV absorption at $254 \mathrm{~nm}$. Silica gel 60 (40-63 $\mu \mathrm{m}$, Merck) was used for column chromatography. Technical solvents were distilled before use. RP-HPLC analyses were performed using an Omnicrom YMC column (4.6 $\left.\mathrm{mm} \times 250 \mathrm{~mm}, 5 \mu \mathrm{m} \mathrm{C}_{18}, 1 \mathrm{~mL} / \mathrm{min}\right)$. The eluent was a linear gradient from water $(0.1 \% \mathrm{TFA})$ to acetonitrile (0.1\% TFA) over 30 minutes and detection at $220 \mathrm{~nm}$ and $254 \mathrm{~nm}$. The retention time $\left(R_{\mathrm{t}}\right)$ of the analytical RP-HPLC is given in minutes with the gradient in percentage of acetonitrile. NMR spectra were obtained at $300 \mathrm{~K}$. The chemical shifts are reported in ppm on the $\delta$ scale relative to the solvent signal used. 4-Hydroxynaphthalene-1-carbaldehyde was purchased from Aldrich, [(S,S)-(COD)Et-DuPHOS-Rh(I)] OTf was purchased from Strem Chemicals and acylase I (from Aspergillus melleus) was puchased from Aldrich.

(S)- $N^{\alpha}$-tert-Butyloxycarbonyl-m-iodotyrosine (5). To a solution of $m$-iodo-L-tyrosine (4) (2.16 g, $7.02 \mathrm{mmol}, 1.0$ eq.) and $\mathrm{NEt}_{3}\left(1.46 \mathrm{~mL}, 10.5 \mathrm{mmol}, 1.5\right.$ eq.) in $100 \mathrm{~mL}$ of $\operatorname{dioxan} / \mathrm{H}_{2} \mathrm{O}(1: 1)$ at $0^{\circ} \mathrm{C}$ ditert-butyldicarbonate (1.69 g, $7.73 \mathrm{mmol}, 1.1 \mathrm{eq.})$ was added in small portions and the mixture was stirred for $45 \mathrm{~min}$ at $0^{\circ} \mathrm{C}$ and additional $18 \mathrm{~h}$ at room temperature. After the dioxane was removed on a Rotary Evaporator, the solution was acidified by addition of a 10\% aqueous solution of citric acid (200 $\mathrm{mL})$ and the aqueous layer was extracted with EtOAc $(3 \times 100 \mathrm{~mL})$. The combined organic layers were dried $\left(\mathrm{Na}_{2} \mathrm{SO}_{4}\right)$ and the solvent removed under reduced pressure to give $\mathbf{5}(2.74 \mathrm{~g}, 96 \%)$ as a colorless oil which was used without further purification. A small sample was purified by flash chromatography for characterization yielding a colorless solid. $R_{\mathrm{f}}=0.15($ EtOAc/hexane $1: 4,1 \% \mathrm{AcOH}) ;[\alpha]_{\mathrm{D}}^{23}+2.4$ (c $\left.0.1, \mathrm{CHCl}_{3}\right) ; \mathrm{mp} 83-85^{\circ} \mathrm{C}$ (lit. $\left.{ }^{1} \mathrm{mp} 84-85^{\circ} \mathrm{C}\right) ;{ }^{1} \mathrm{H} \mathrm{NMR}\left(360 \mathrm{MHz}, \mathrm{MeOH}-\mathrm{d}_{4}\right) \delta 7.53(\mathrm{~d}, J=1.31 \mathrm{~Hz}$, 1H), $7.03(\mathrm{dd}, J=8.25,2.10 \mathrm{~Hz}, 1 \mathrm{H}), 6.73(\mathrm{~d}, J=8.23 \mathrm{~Hz}, 1 \mathrm{H}), 4.25(\mathrm{dd}, J=8.63,5.03 \mathrm{~Hz}, 1 \mathrm{H}), 3.02$ (dd, J=13.92, $4.97 \mathrm{~Hz}, 1 \mathrm{H}), 2.76(\mathrm{dd}, J=13.80,8.92 \mathrm{~Hz}, 1 \mathrm{H}), 1.38(\mathrm{~s}, 9 \mathrm{H}) ;{ }^{13} \mathrm{C}-\mathrm{NMR}(90 \mathrm{MHz}$, 
MeOH-d $\left.{ }_{4}\right) \delta 175.2,157.7,156.8,141.0,140.9,131.4131 .4,115.6,84.4,80.6,56.3,37.3,28.7 ;$ MS (ESI) $\mathrm{m} / \mathrm{z} 308.1[\mathrm{M}+\mathrm{H}-\mathrm{Boc}]^{+}, 852.9[2 \mathrm{M}+\mathrm{K}]^{+}, 1243.8[3 \mathrm{M}+\mathrm{Na}]^{+}, 1259.7[3 \mathrm{M}+\mathrm{K}]^{+}$.

\section{Synthesis of 3-aryl-substituted tyrosine analogues $7 \mathrm{~b}-\mathrm{j}$. General procedure.}

To a solution of 6 (1.0 eq.) in degassed DME/ $\mathrm{H}_{2} \mathrm{O}(6: 1 ; 7 \mathrm{~mL} / \mathrm{mmol})$ was added the appropriate aryl boronic acid (1.5 eq.) and $\mathrm{Na}_{2} \mathrm{CO}_{3}$ (2.0 eq.). After five vacuum/argon cycles $\mathrm{Pd}(\mathrm{OAc})_{2}(5 \mathrm{~mol} \%)$ and $\mathrm{P}(o \text {-tolyl })_{3}(10 \mathrm{~mol} \%)$ were added and the mixture heated to $80^{\circ} \mathrm{C}$ until completed conversion $(3-8 \mathrm{~h})$. After the reaction mixture was cooled to room temperature, it was passed through a short column with a bottom layer of silica gel $(40-63 \mu \mathrm{m})$ and a top layer of $\mathrm{NaHCO}_{3}$ using EtOAc as eluent. The solvent was removed under reduced pressure and the crude product purified by flash chromatography using an appropriate mixture of EtOAc and hexane as eluent.

$(S)$ - $N^{a}$-tert-Butyloxycarbonyl-O-benzyl-m-(p-tolyl)tyrosine benzyl ester (7b). Pale yellow solid;

Yield 93\%; $R_{\mathrm{f}}=0.25(\mathrm{EtOAc} / \mathrm{hexane}, 1: 4) ;[\alpha]_{\mathrm{D}}{ }^{23}+3.0\left(\mathrm{c} 1.3, \mathrm{CHCl}_{3}\right) ; \mathrm{mp} 97-101^{\circ} \mathrm{C} ;{ }^{1} \mathrm{H}-\mathrm{NMR}(250$ $\left.\mathrm{MHz}, \mathrm{CDCl}_{3}\right) \delta 7.45-7.38(\mathrm{~m}, 2 \mathrm{H}), 7.34-7.16(\mathrm{~m}, 12 \mathrm{H}), 7.06(\mathrm{~d}, J=1.3 \mathrm{~Hz}, 1 \mathrm{H}), 6.89(\mathrm{~d}, J=8.5 \mathrm{~Hz}$, 1H), $6.82(\mathrm{~d}, J=8.4 \mathrm{~Hz}, 1 \mathrm{H}), 5.12(\mathrm{~d}, J=12.3 \mathrm{~Hz}, 1 \mathrm{H}), 5.06(\mathrm{~d}, J=12.4 \mathrm{~Hz}, 1 \mathrm{H}), 5.05-4.95(\mathrm{~m}, 3 \mathrm{H})$, $4.60(\mathrm{~m}, 1 \mathrm{H}), 3.13-2.90(\mathrm{~m}, 2 \mathrm{H}), 2.36(\mathrm{~s}, 3 \mathrm{H}), 1.39(\mathrm{~s}, 9 \mathrm{H}) ;{ }^{13} \mathrm{C} \mathrm{NMR}\left(63 \mathrm{MHz}, \mathrm{CDCl}_{3}\right) \delta 171.8$, 155.0, 154.7, 137.2, 136.6, 135.2, 135.1, 131.9, 131.1, 129.4, 128.9, 128.6, 128.5, 128.42, 128.37, $127.5,126.8,113.4,79.9,70.4,67.1,54.51,37.4,28.3,21.2$; HRMS (EI) calcd for $\mathrm{C}_{35} \mathrm{H}_{37} \mathrm{NO}_{5}$ 551.26715; found 551.26702.

$(S)$ - $N^{\alpha}$-tert-Butyloxycarbonyl- $O$-benzyl-m-( $m$-tolyl)tyrosine benzyl ester $(7 \mathrm{c})$. Pale yellow solid; Yield 79\%; $R_{\mathrm{f}}=0.25($ EtOAc/hexane, $1: 4) ;[\alpha]_{\mathrm{D}}{ }^{23}+2.9\left(\mathrm{c} 2.8, \mathrm{CHCl}_{3}\right) ; \mathrm{mp} 78-82^{\circ} \mathrm{C} ;{ }^{1} \mathrm{H}-\mathrm{NMR}(250$ $\left.\mathrm{MHz}, \mathrm{CDCl}_{3}\right) \delta 7.36-7.21(\mathrm{~m}, 13 \mathrm{H}), 7.14-7.04(\mathrm{~m}, 2 \mathrm{H}), 6.92(\mathrm{~d}, J=8.7 \mathrm{~Hz}, 1 \mathrm{H}), 6.85(\mathrm{~d}, J=8.3 \mathrm{~Hz}$, 1H), $5.13(\mathrm{~d}, J=12.2 \mathrm{~Hz}, 1 \mathrm{H}), 5.07(\mathrm{~d}, J=12.6 \mathrm{~Hz}, 1 \mathrm{H}), 5.05-4.99(\mathrm{~m}, 3 \mathrm{H}), 4.61(\mathrm{~m}, 1 \mathrm{H}), 3.12-2.98(\mathrm{~m}$, 2H), $2.36(\mathrm{~s}, 3 \mathrm{H}), 1.39(\mathrm{~s}, 9 \mathrm{H}) ;{ }^{13} \mathrm{C} \mathrm{NMR}\left(63 \mathrm{MHz}, \mathrm{CDCl}_{3}\right) \delta 171.8,155.1,154.7,138.0,137.3,137.2$, 135.1, 132.0, 131.4, 130.3, 129.1, 128.53, 128.45, 128.37, 127.8, 127.7, 127.5, 126.8, 126.7, 113.4, 
80.0, 70.5, 67.1, 54.6, 37.4, 28.3, 28.1, 21.5; HRMS (EI) calcd for $\mathrm{C}_{35} \mathrm{H}_{37} \mathrm{NO}_{5}$ 551.26715; found 551.26682.

(S)- $N^{\alpha}$-tert-Butyloxycarbonyl-O-benzyl-m-(o-tolyl)tyrosine benzyl ester (7d). Pale yellow oil; Yield 70\%; $R_{\mathrm{f}}=0.25($ EtOAc/hexane, $1: 4) ;[\alpha]_{\mathrm{D}}{ }^{23}+2.6\left(\mathrm{c} 1.9, \mathrm{CHCl}_{3}\right) ;{ }^{1} \mathrm{H} \mathrm{NMR}\left(250 \mathrm{MHz}, \mathrm{CDCl}_{3}\right) \delta$ 7.30-7.20 (m, 10H), 7.20-7.17 (m, 2H), 7.17-7.12 (m, 2H), 6.93 (d, J=8.5 Hz, 1H), 6.90-6.81 (m, 2H), $5.14(\mathrm{~d}, J=12.2 \mathrm{~Hz}, 1 \mathrm{H}), 5.08(\mathrm{~d}, J=12.4 \mathrm{~Hz}, 1 \mathrm{H}) 5.03-4.95(\mathrm{~m}, 3 \mathrm{H}), 4.60(\mathrm{~m}, 1 \mathrm{H}), 3.13-2.92(\mathrm{~m}, 2 \mathrm{H})$, $1.39(\mathrm{~s}, 9 \mathrm{H}) ;{ }^{13} \mathrm{C} \mathrm{NMR}\left(63 \mathrm{MHz}, \mathrm{CDCl}_{3}\right) \delta 171.7,155.0,154.8,138.3,137.3,136.6,135.1,132.2$, $131.7,130.0,129.5,129.2,128.5,128.4,128.3,128.1,127.4,127.3,126.5,125.3,113.2,79.9,70.3$, 67.1, 54.5, 37.4, 28.3, 20.1; HRMS (EI) calcd for $\mathrm{C}_{35} \mathrm{H}_{37} \mathrm{NO}_{5}$ 551.26715; found 551.26764.

(S)- $N^{\alpha}$-tert-Butyloxycarbonyl-O-benzyl-m-(3-hydroxyphenyl)tyrosine benzyl ester (7e). Pale

yellow solid; Two rotamers (ca. 4:1 ratio); Yield 80\%; $R_{\mathrm{f}}=0.21($ EtOAc/hexane, $1: 4) ;[\alpha]_{\mathrm{D}}^{23}+3.8(\mathrm{c} 0.9$, $\left.\mathrm{CHCl}_{3}\right) ; \mathrm{mp} 65-68^{\circ} \mathrm{C} ;{ }^{1} \mathrm{H} \mathrm{NMR}\left(250 \mathrm{MHz}, \mathrm{CDCl}_{3}\right) \delta 7.33-7.15(\mathrm{~m}, 11 \mathrm{H}), 7.11-6.95(\mathrm{~m}, 3 \mathrm{H}), 6.89(\mathrm{dd}$, $J=8.3,1.7 \mathrm{~Hz}, 1 \mathrm{H}), 6.82(\mathrm{~s}, 1 \mathrm{H}), 6.78(\mathrm{~m}, 1 \mathrm{H}), 5.53(\mathrm{~s}, 1 \mathrm{H}), 5.15-5.00(\mathrm{~m}, 3 \mathrm{H}), 4.98(\mathrm{~s}, 2 \mathrm{H}), 4.60(\mathrm{~m}$, $1 \mathrm{H}(80 \%)), 4.60(\mathrm{~m}, 1 \mathrm{H}(20 \%)), 3.11-2.90(\mathrm{~m}, 2 \mathrm{H}), 1.38(\mathrm{~s}, 9 \mathrm{H}(80 \%)), 1.29(\mathrm{~s}, 9 \mathrm{H}(20 \%)) ;{ }^{13} \mathrm{C}$ NMR $\left(63 \mathrm{MHz}, \mathrm{CDCl}_{3}\right) \delta 171.8,155.4,155.2,154.6,139.6,137.1,135.0,131.8,130.9,129.2,128.9,128.5$, 128.3, 127.5, 126.7, 121.8, 116.5, 113.9, 113.5, 80.1, 70.5, 67.1, 54.5, 37.3, 28.2; HRMS (EI) calcd for $\mathrm{C}_{34} \mathrm{H}_{35} \mathrm{NO}_{6}$ 553.24644; found 553.24703.

(S)- $N^{\alpha}$-tert-Butyloxycarbonyl-O-benzyl-m-(3-chlorophenyl)tyrosine benzyl ester (7f). Pale yellow oil; Yield 39\%; $R_{\mathrm{f}}=0.38($ EtOAc/hexane, $1: 4) ;[\alpha]_{\mathrm{D}}{ }^{23}+3.0\left(\mathrm{c} 0.5, \mathrm{CHCl}_{3}\right) ;{ }^{1} \mathrm{H}$ NMR $\left(250 \mathrm{MHz}, \mathrm{CDCl}_{3}\right)$ $\delta 7.55(\mathrm{~m}, 1 \mathrm{H}), 7.42-7.22(\mathrm{~m}, 13 \mathrm{H}), 7.05(\mathrm{~d}, J=1.7 \mathrm{~Hz}, 1 \mathrm{H}), 6.98(\mathrm{~d}, J=8.3 \mathrm{~Hz}, 1 \mathrm{H}), 6.88(\mathrm{~d}, J=8.3 \mathrm{~Hz}$, 1H), 5.17 (d, J=12.3 Hz, 2H), $5.10(\mathrm{~d}, J=12.5 \mathrm{~Hz}, 2 \mathrm{H}), 5.04-4.97(\mathrm{~m}, 3 \mathrm{H}), 4.64(\mathrm{~m}, 1 \mathrm{H}), 3.20-2.92(\mathrm{~m}$, 2H), $1.42(\mathrm{~s}, 9 \mathrm{H}) ;{ }^{13} \mathrm{C}$ NMR (63 MHz, $\left.\mathrm{CDCl}_{3}\right) \delta 171.7,155.0,154.5,139.9,136.9,135.1,133.7$, $131.8,129.9,129.7,129.1,128.6,128.54,128.46,128.4,127.73,127.68,127.0,126.8,113.3,80.0$, 70.5, 67.1, 54.5, 37.4, 28.3; HRMS (EI) calcd for $\mathrm{C}_{34} \mathrm{H}_{34} \mathrm{ClNO}_{5}$ 571.21255; found 571.21109. 
(S)- $N^{\alpha}$-tert-Butyloxycarbonyl-O-benzyl-m-(4-fluorophenyl)tyrosine benzyl ester (7g). Pale yellow solid; Yield 94\%; $R_{\mathrm{f}}=0.25\left(\right.$ EtOAc/hexane, 1:4); $[\alpha]_{\mathrm{D}}{ }^{23}+1.3\left(\mathrm{c} 1.7, \mathrm{CHCl}_{3}\right) ; \mathrm{mp} 108-110^{\circ} \mathrm{C} ;{ }^{1} \mathrm{H}$ NMR $\left(250 \mathrm{MHz}, \mathrm{CDCl}_{3}\right) \delta$ 7.54-7.45 (m, 2H), 7.36-7.25 (m, 10H), 7.12-7.03 (m, 3H), $6.96(\mathrm{~d}$, $J=8.4 \mathrm{~Hz}, 1 \mathrm{H}), 6.88(\mathrm{~d}, J=8.4 \mathrm{~Hz}, 1 \mathrm{H}), 5.17(\mathrm{~d}, J=12.2 \mathrm{~Hz}, 1 \mathrm{H}), 5.10(\mathrm{~d}, J=12.4 \mathrm{~Hz}, 1 \mathrm{H}), 5.04(\mathrm{~m}, 3 \mathrm{H})$, $4.64(\mathrm{~m}, 1 \mathrm{H}), 3.20-2.95(\mathrm{~m}, 2 \mathrm{H}), 1.42(\mathrm{~s}, 9 \mathrm{H}) ;{ }^{13} \mathrm{C} \mathrm{NMR}\left(63 \mathrm{MHz}, \mathrm{CDCl}_{3}\right) \delta 171.7,163.9,160.0$, $155.0,154.6,137.0,135.1,134.11,134.06,131.9,131.2,131.1,130.2,129.4,128.5,128.43,128.37$, $127.7,126.8,114.9,114.6,113.3,79.9,70.5,67.1,54.5,37.4,28.3$; HRMS (EI) calcd for $\mathrm{C}_{34} \mathrm{H}_{34} \mathrm{FNO}_{5}$ 555.24210; found 555.24184.

(S)-N $N^{\alpha}$-tert-Butyloxycarbonyl-O-benzyl-m-(4-carboxyphenyl)tyrosine benzyl ester (7h). Pale yellow solid; Two rotamers (ca. 7:3 ratio); Yield 92\%; $R_{\mathrm{f}}=0.35($ EtOAc/hexane 1:4, $1 \% \mathrm{AcOH}) ;[\alpha]_{\mathrm{D}}{ }^{23}$ -10.5 (c 0.02, $\left.\mathrm{CHCl}_{3}\right) ;{ }^{1} \mathrm{H}$ NMR (250 MHz, $\left.\mathrm{CDCl}_{3}\right) \delta 9.53(\mathrm{~s}, 1 \mathrm{H}), 8.12(\mathrm{~d}, J=7.9 \mathrm{~Hz}, 2 \mathrm{H}), 7.63(\mathrm{~d}$, $J=8.0 \mathrm{~Hz}, 2 \mathrm{H}), 7.39-7.22(\mathrm{~m}, 10 \mathrm{H}), 7.11(\mathrm{~s}, 1 \mathrm{H}), 6.99(\mathrm{~d}, J=8.4 \mathrm{~Hz}, 1 \mathrm{H}), 6.90(\mathrm{~d}, J=8.2 \mathrm{~Hz}, 1 \mathrm{H}), 5.20-$ $5.08(\mathrm{~m}, 2 \mathrm{H}), 5.08-5.04(\mathrm{~m}, 3 \mathrm{H}), 4.66(\mathrm{~m}, 1 \mathrm{H}(70 \%)), 4.38(\mathrm{~m}, 1 \mathrm{H}(30 \%)), 3.20-3.01(\mathrm{~m}, 2 \mathrm{H}), 1.42(\mathrm{~s}$, 9H (70\%)), $1.32(\mathrm{~s}, 9 \mathrm{H}(30 \%)) ;{ }^{13} \mathrm{C} \mathrm{NMR}\left(125 \mathrm{MHz}, \mathrm{CDCl}_{3}\right) \delta 171.8,171.3,155.1,154.7,143.9$, $136.8,135.1,132.0,131.9,129.93,129.88,129.7,129.6,128.7,128.6,128.5,127.8,127.7,126.9$, 126.8, 113.3, 80.1, 70.6, 67.2, 54.5, 37.5, 28.3; MS (ESI) m/z 604.4 [M+Na $]^{+}, 1185.1[2 \mathrm{M}+\mathrm{Na}]^{+}$.

$(S)-N^{\alpha}$-tert-Butyloxycarbonyl- $O$-benzyl-m-(2-furanyl)tyrosine benzyl ester (7i). Pale yellow solid; Yield 99\%; $R_{\mathrm{f}}=0.38($ EtOAc/hexane, $1: 4) ;[\alpha]_{\mathrm{D}}{ }^{23}+7.1\left(\mathrm{c} 0.3, \mathrm{CHCl}_{3}\right) ; \mathrm{mp} 97-100^{\circ} \mathrm{C} ;{ }^{1} \mathrm{H}$ NMR $(250$ $\left.\mathrm{MHz}, \mathrm{CDCl}_{3}\right) \delta 7.67(\mathrm{~d}, J=0.4 \mathrm{~Hz}, 1 \mathrm{H}), 7.52-7.36(\mathrm{~m}, 6 \mathrm{H}), 7.35-7.30(\mathrm{~m}, 5 \mathrm{H}), 6.93-6.86(\mathrm{~m}, 3 \mathrm{H}), 6.44$ (dd, J=3.4, $1.8 \mathrm{~Hz}, 1 \mathrm{H}), 5.17-5.12(\mathrm{~m}, 4 \mathrm{H}), 5.05$ (d, J=7.0 Hz, 1H), $4.64(\mathrm{~m}, 1 \mathrm{H}), 3.17-3.01(\mathrm{~m}, 2 \mathrm{H})$, $1.44(\mathrm{~s}, 9 \mathrm{H}) ;{ }^{13} \mathrm{C}$ NMR (63 MHz, $\left.\mathrm{CDCl}_{3}\right) \delta 171.7,155.0,153.5,149.9,141.0,136.7,135.2,128.61$, $128.57,128.49,128.43,128.3,128.2,128.1,127.6,126.9,120.1,112.3,111.8,110.3,79.9,70.5,67.1$, 54.6, 54.6, 37.5, 28.3; HRMS (EI) calcd for $\mathrm{C}_{32} \mathrm{H}_{33} \mathrm{NO}_{6}$ 527.23079; found 527.23086.

(S)- $N^{\alpha}$-tert-Butyloxycarbonyl-O-benzyl-m-(3-thienyl)tyrosine benzyl ester (7j). Pale yellow solid; Yield 45\%; $R_{\mathrm{f}}=0.37($ EtOAc/hexane, $1: 4) ;[\alpha]_{\mathrm{D}}{ }^{23} \pm 0.0\left(\mathrm{c} 1.2, \mathrm{CHCl}_{3}\right) ;$ mp $115-117^{\circ} \mathrm{C} ;{ }^{1} \mathrm{H}$ NMR $(250$ 
$\left.\mathrm{MHz}, \mathrm{CDCl}_{3}\right) \delta 7.57(\mathrm{dd}, J=3.0,1.3 \mathrm{~Hz}, 1 \mathrm{H}), 7.41(\mathrm{dd}, J=5.1,1.3 \mathrm{~Hz}, 1 \mathrm{H}), 7.39-7.34(\mathrm{~m}, 4 \mathrm{H}), 7.34-$

$7.23(\mathrm{~m}, 8 \mathrm{H}), 6.95-6.84(\mathrm{~m}, 2 \mathrm{H}), 5.15(\mathrm{~d}, J=12.2 \mathrm{~Hz}, 1 \mathrm{H}), 5.09$ (d, J=12.3 Hz, 1H), 5.07 (s, $2 \mathrm{H}), 5.02$

$(\mathrm{m}, 1 \mathrm{H}), 4.63(\mathrm{~m}, 1 \mathrm{H}), 3.17-2.96(\mathrm{~m}, 2 \mathrm{H}), 1.41(\mathrm{~s}, 9 \mathrm{H}) ;{ }^{13} \mathrm{C} \mathrm{NMR}\left(63 \mathrm{MHz}, \mathrm{CDCl}_{3}\right) \delta 171.7,155.0$, $154.7,137.9,136.9,135.1,130.8,128.9,128.52,128.49,128.45,128.37,127.8,127.1,125.5,124.3$, 123.4, 113.2, 79.9, 70.6, 67.1, 54.5, 37.4, 28.3; HRMS (EI) calcd for $\mathrm{C}_{32} \mathrm{H}_{33} \mathrm{NO}_{5} \mathrm{~S}$ 543.20794; found 543.20752.

$(S)-N^{\alpha}$-tert-Butyloxycarbonyl-O-benzyl-m-(o-tolyl)tyrosine $(\mathbf{8 b})$. A solution of lithium hydroxide (4.7 mg, $112 \mu \mathrm{mol}, 1.0$ eq.) in $\mathrm{H}_{2} \mathrm{O}(0.17 \mathrm{~mL})$ was added to a solution of $7 \mathbf{d}$ (62 mg, $112 \mu \mathrm{mol}, 1.0$ eq.) in $2 \mathrm{~mL}$ of $\mathrm{THF}$ at $0^{\circ} \mathrm{C}$. After stirring for $18 \mathrm{~h}$, a $10 \%$ aqueous solution of citric acid $(50 \mathrm{~mL})$ was added and the aqueous layer was extracted with EtOAc $(3 \times 30 \mathrm{~mL})$. The combined organic layers were dried $\left(\mathrm{Na}_{2} \mathrm{SO}_{4}\right)$, the solvent removed under reduced pressure and the residue purified by flash chromatography on silica gel (EtOAc/hexane 1:1, 1\% AcOH) yielding $\mathbf{8 b}(43 \mathrm{mg}, 83 \%)$ as a colorless solid. $R_{\mathrm{f}}=0.30($ EtOAc/hexane $1: 1,1 \% \mathrm{AcOH}) ;[\alpha]_{\mathrm{D}}{ }^{23}+24.1\left(\mathrm{c} 0.9, \mathrm{CHCl}_{3}\right) ; \mathrm{mp} 68-72{ }^{\circ} \mathrm{C} ;{ }^{1} \mathrm{H} \mathrm{NMR}$ $\left(360 \mathrm{MHz}, \mathrm{CDCl}_{3}\right) \delta 7.32-7.16(\mathrm{~m}, 9 \mathrm{H}), 7.11(\mathrm{dd}, J=8.4,1.8 \mathrm{~Hz}, 1 \mathrm{H}), 7.00(\mathrm{~s}, 1 \mathrm{H}), 6.94(\mathrm{~d}, J=8.4 \mathrm{~Hz}$, 1H), $5.01(\mathrm{~s}, 2 \mathrm{H}), 4.96(\mathrm{~m}, 1 \mathrm{H}), 4.60(\mathrm{~m}, 1 \mathrm{H}), 3.16(\mathrm{dd}, J=14.2,3.9 \mathrm{~Hz}, 1 \mathrm{H}), 3.04(\mathrm{dd}, J=14.0,5.6 \mathrm{~Hz}$, 1H), $2.18(\mathrm{~s}, 3 \mathrm{H}), 1.42(\mathrm{~s}, 9 \mathrm{H}) ;{ }^{13} \mathrm{C} \mathrm{NMR}\left(90 \mathrm{MHz}, \mathrm{CDCl}_{3}\right) \delta 176.1,155.0,138.3,137.3,136.7,132.2$, $131.9,130.0,129.6,129.2,128.3,127.5,127.3,126.6,125.3,113.4,80.3,70.4,54.4,36.9,28.3,20.1$; HRMS (EI) calcd for $\mathrm{C}_{28} \mathrm{H}_{31} \mathrm{NO}_{5}$ 461.22022; found 461.22022.

(S)- $N^{\alpha}$-tert-Butyloxycarbonyl-m-(o-tolyl)tyrosine (9b). Palladium on charcoal (5\% Pd/C, $0.11 \mathrm{~g}$, $10 \mathrm{~mol} \% \mathrm{Pd})$ was added to a degassed solution of $7 \mathbf{b}(0.30 \mathrm{~g}, 0.56 \mathrm{mmol})$ in $N, N-$ dimethylacetamide/MeOH $(1: 1,15 \mathrm{~mL})$. Hydrogenation was carried out at 1 bar hydrogen pressure for $6 \mathrm{~h}$. The catalyst was removed by filtration, the solvent removed under reduced pressure and the residue purified by flash chromatography on silica gel (EtOAc/hexane 1:2, 1\% AcOH) yielding $9 b$ (179 mg, 
$87 \%)$ as a colorless solid. $R_{\mathrm{f}}=0.27(\mathrm{EtOAc} / \mathrm{hexane} 1: 1,1 \% \mathrm{AcOH}) ;[\alpha]_{\mathrm{D}}{ }^{23}+21.9(\mathrm{c} 0.6, \mathrm{MeOH}) ; \mathrm{mp} 82-$ $85^{\circ} \mathrm{C} ;{ }^{1} \mathrm{H}$ NMR $\left(360 \mathrm{MHz}\right.$, acetone-d $\left.\mathrm{d}_{6}\right) \delta 7.26-7.20(\mathrm{~m}, 2 \mathrm{H}), 7.20-7.14(\mathrm{~m}, 2 \mathrm{H}), 7.11(\mathrm{dd}, J=8.3,1.7$ $\mathrm{Hz}, 1 \mathrm{H}), 6.97(\mathrm{~d}, J=1.7 \mathrm{~Hz}, 1 \mathrm{H}), 6.90(\mathrm{~d}, J=8.2 \mathrm{~Hz}, 1 \mathrm{H}), 4.42(\mathrm{~m}, 1 \mathrm{H}), 3.15(\mathrm{dd}, J=13.9,5.0 \mathrm{~Hz}, 1 \mathrm{H})$, $2.97(\mathrm{dd}, J=13.7,8.2 \mathrm{~Hz}, 1 \mathrm{H}), 2.18(\mathrm{~s}, 3 \mathrm{H}), 1.36(\mathrm{~s}, 9 \mathrm{H}) ;{ }^{13} \mathrm{C}$ NMR $\left(90 \mathrm{MHz}\right.$, acetone-d $\left.\mathrm{d}_{6}\right) \delta 174.5$, 157.0, 154.7, 140.5, 138.6, 133.7, 132.0, 131.4, 131.1, 130.4, 129.9, 128.9, 127.1, 117.2, 80.2, 56.6, 38.3, 21.2; HRMS (EI) calcd for $\mathrm{C}_{21} \mathrm{H}_{25} \mathrm{NO}_{5} 371,17327$; found 371.17221 .

\section{4-(tert-Butyldimethylsilyloxy)naphthalene-1-carbaldehyde $\quad$ (12). A solution $\quad$ of 4-} hydroxynaphthalene-1-carbaldehyde (11) (1.13 g, $6.56 \mathrm{mmol}, 1.0$ eq.) in dry THF (30 mL) was cooled to $0^{\circ} \mathrm{C}$ and sequentially imidazole $(0.86 \mathrm{~g}, 12.6 \mathrm{mmol}, 1.9$ eq. $)$ and tert-butyldimethylsilyl chloride (TBSCl) (1.72 g, $11.4 \mathrm{mmol}, 1.7$ eq.) were added. After stirring at room temperature for $18 \mathrm{~h}$ the reaction mixture was filtered and the solvent evaporated. The residue was taken up in EtOAc $(100 \mathrm{~mL})$ and washed with saturated aqueous $\mathrm{NH}_{4} \mathrm{Cl}(40 \mathrm{~mL})$, water $(40 \mathrm{~mL})$ and brine $(40 \mathrm{~mL})$ and dried over $\mathrm{Na}_{2} \mathrm{SO}_{4}$. The solvent was removed under reduced pressure and the residue purified by flash chromatography on silica gel (EtOAc/hexane 1:20) yielding $12(1.69 \mathrm{~g}, 88 \%)$ as a pale yellow solid. $R_{\mathrm{f}}=0.26\left(\right.$ EtOAc/hexane, 1:10); mp 87-91 ${ }^{\circ} \mathrm{C} ;{ }^{1} \mathrm{H}$ NMR $\left(500 \mathrm{MHz}, \mathrm{CDCl}_{3}\right) \delta 10.23(\mathrm{~s}, 1 \mathrm{H}), 9.31(\mathrm{~d}$, $J=8.5 \mathrm{~Hz}, 1 \mathrm{H}), 8.28(\mathrm{~d}, J=8.4 \mathrm{~Hz}, 1 \mathrm{H}), 7.87(\mathrm{~d}, J=7.9 \mathrm{~Hz}, 1 \mathrm{H}), 7.69(\mathrm{dd}, J=7.4,7.4 \mathrm{~Hz}, 1 \mathrm{H}), 7.58(\mathrm{dd}$, $J=7.6,7.6 \mathrm{~Hz}, 1 \mathrm{H}), 6.95(\mathrm{~d}, J=7.9 \mathrm{~Hz}, 1 \mathrm{H}), 1.11(\mathrm{~s}, 9 \mathrm{H}), 0.37(\mathrm{~s}, 6 \mathrm{H}) ;{ }^{13} \mathrm{C} \mathrm{NMR}\left(63 \mathrm{MHz}, \mathrm{CDCl}_{3}\right) \delta$ 192.1, 158.0, 139.0, 132.5, 129.4, 127.7, 126.2, 125.2, 124.9, 122.9, 111.3, 25.7, 18.4, -4.2; HRMS (EI) calcd for $\mathrm{C}_{17} \mathrm{H}_{22} \mathrm{O}_{2} \mathrm{Si} 286.13892$; found 286.13885 .

Synthesis of Dipeptides 10a-d, 16 and 17. General Procedure. Peptide synthesis was carried out using TCP-resin following standard Fmoc-strategy. ${ }^{1}$ Fmoc-Val-OH (21 mg, $\left.0.06 \mathrm{mmol}\right)$ was attached to the TCP resin $(50 \mathrm{mg})$ with DIEA $(25 \mu \mathrm{L})$ in anhydrous DCM $(1.5 \mathrm{~mL})$ at room temperature for $1 \mathrm{~h}$, followed by addition of $\mathrm{MeOH}(0.1 \mathrm{~mL})$ for 15 minutes for quenching, yielding $62 \mathrm{mg}$ of Fmoc-Val- 
TCP resin (substitution level $0.71 \mathrm{mmol} / \mathrm{g}$ resin). For Fmoc-deprotection the resin was treated with $20 \%$ piperidine in NMP (v/v) and washed with NMP $(5 \times 1 \mathrm{~min})$. The coupling of side chain unprotected tyrosine analogues was achieved using 1.8 eq. $(64 \mu \mathrm{mol})$ of the corresponding amino acid, 1.8 eq. TBTU $(21 \mathrm{mg}, 64 \mu \mathrm{mol}), 1.8$ eq. HOBt $(8.6 \mathrm{mg}, 64 \mu \mathrm{mol})$ and 10 eq. collidine $(47 \mu \mathrm{L}, 0.35 \mathrm{mmol})$ in NMP $(1 \mathrm{~mL})$ for $30 \mathrm{~min}$ at room temperature after which the resin was washed with NMP $(5 \times 1 \mathrm{~min})$ and DCM $(3 \times 1 \mathrm{~min})$. The dipeptides were deprotected and cleaved from resin by treatment with TFA/DCM/ $\mathrm{H}_{2} \mathrm{O}(50: 40: 10, \mathrm{v} / \mathrm{v})$ for $1 \mathrm{~h}$. The solvents were evaporated to dryness to give the pure dipeptides as colorless solids.

H-m -(phenyl)Tyr-Val-OH (10a). 98\% purity; RP-HPLC $(10 \rightarrow 50 \%) t_{\mathrm{R}}=18.5 ;{ }^{1} \mathrm{H} \mathrm{NMR}(500 \mathrm{MHz}$, $\left.\mathrm{MeOH}-\mathrm{d}_{4}\right) \delta 7.54(\mathrm{~d}, J=7.5 \mathrm{~Hz}, 2 \mathrm{H}), 7.35(\mathrm{dd}, J=7.6,7.6 \mathrm{~Hz}, 2 \mathrm{H}), 7.26(\mathrm{dd}, J=7.4,7.4 \mathrm{~Hz}, 1 \mathrm{H}), 7.23$ $(\mathrm{d}, J=2.0 \mathrm{~Hz}, 1 \mathrm{H}), 7.10(\mathrm{dd}, J=8.5,2.1 \mathrm{~Hz}, 1 \mathrm{H}), 6.87(\mathrm{~d}, J=8.2 \mathrm{~Hz}, 1 \mathrm{H}), 4.37(\mathrm{~d}, J=5.2 \mathrm{~Hz}, 1 \mathrm{H}), 4.16$ (dd, $J=8.6,5.0 \mathrm{~Hz}, 1 \mathrm{H}), 3.24(\mathrm{dd}, J=14.6,4.9 \mathrm{~Hz}, 1 \mathrm{H}), 2.96(\mathrm{dd}, J=14.5,8.7 \mathrm{~Hz}, 1 \mathrm{H}), 2.21(\mathrm{~m}, 1 \mathrm{H})$, $0.98(\mathrm{~d}, J=6.7 \mathrm{~Hz}, 3 \mathrm{H}), 0.97$ (d, $J=6.7 \mathrm{~Hz}, 3 \mathrm{H}) ;{ }^{13} \mathrm{C} \mathrm{NMR}\left(125 \mathrm{MHz}, \mathrm{MeOH}-\mathrm{d}_{4}\right) \delta 174.2,170.1,155.3$, $139.9,132.9,130.59,130.56,130.5,129.0,127.9,126.4,117.6,59.355 .8,37.9,31.8,19.6,18.2$; MS (ESI) $\mathrm{m} / \mathrm{z} 357.2[\mathrm{M}+\mathrm{H}]^{+}, 713.1[\mathrm{M}+\mathrm{Na}]^{+}$.

H-m-(o-tolyl)Tyr-Val-OH (10b). $98 \%$ purity; RP-HPLC $(10 \rightarrow 50 \%) t_{\mathrm{R}}=19.8 ;{ }^{1} \mathrm{H} \mathrm{NMR}(500 \mathrm{MHz}$, MeOH-d $\left.{ }_{4}\right) \delta 7.21-7.17(\mathrm{~m}, 2 \mathrm{H}), 7.17-7.11(\mathrm{~m}, 3 \mathrm{H}) 7.01(\mathrm{~d}, J=2.2 \mathrm{~Hz}, 1 \mathrm{H}), 6.85(\mathrm{~d}, J=8.3 \mathrm{~Hz}, 1 \mathrm{H})$, $4.36(\mathrm{~d}, J=5.2 \mathrm{~Hz}, 1 \mathrm{H}), 4.15(\mathrm{dd}, J=8.6,5.1 \mathrm{~Hz}, 1 \mathrm{H}), 3.22(\mathrm{dd}, J=14.5,5.0 \mathrm{~Hz}, 1 \mathrm{H}), 2.94(\mathrm{dd}, J=14.5$, $8.6 \mathrm{~Hz}, 1 \mathrm{H}), 2.20(\mathrm{~m}, 1 \mathrm{H}), 2.14(\mathrm{~s}, 3 \mathrm{H}), 0.97(\mathrm{~d}, J=6.7 \mathrm{~Hz}, 3 \mathrm{H}), 0.96(\mathrm{~d}, J=6.8 \mathrm{~Hz}, 3 \mathrm{H}) ;{ }^{13} \mathrm{C}$ NMR $\left(125 \mathrm{MHz}, \mathrm{MeOH}-\mathrm{d}_{4}\right) \delta 174.0,170.0,155.2,139.7,138.1,133.2,131.2,131.1,130.6,130.5,128.4$, $126.5,126.0,59.2,55.7,37.8,31.7,20.2,19.5,18.2 ; \mathrm{MS}(\mathrm{ESI}) \mathrm{m} / \mathrm{z} 371.2[\mathrm{M}+\mathrm{H}]^{+}, 741.1[\mathrm{M}+\mathrm{Na}]^{+}$.

H-m-(phenyl)Tyr-D-Val-OH (10c). 95\% purity; RP-HPLC $(10 \rightarrow 50 \%) t_{\mathrm{R}}=21.9 ;{ }^{1} \mathrm{H}$ NMR $(500$ $\left.\mathrm{MHz}, \mathrm{MeOH}-\mathrm{d}_{4}\right) \delta$ 7.25-7.17 (m, 3H), 7.16-7.11 (m, 2H), $7.01(\mathrm{~d}, J=2.2 \mathrm{~Hz}, 1 \mathrm{H}), 6.88(\mathrm{~d}, J=8.2 \mathrm{~Hz}$, 1H), $4.29(\mathrm{~d}, J=5.2 \mathrm{~Hz}, 1 \mathrm{H}), 4.19(\mathrm{dd}, J=7.4,7.4 \mathrm{~Hz}, 1 \mathrm{H}), 3.14(\mathrm{dd}, J=14.0,7.2 \mathrm{~Hz}, 1 \mathrm{H}), 3.02(\mathrm{dd}$, $J=14.0,7.6 \mathrm{~Hz}, 1 \mathrm{H}), 2.11(\mathrm{~m}, 1 \mathrm{H}), 0.86(\mathrm{~d}, J=6.9 \mathrm{~Hz}, 3 \mathrm{H}), 0.83(\mathrm{~d}, J=6.9 \mathrm{~Hz}, 3 \mathrm{H}) ;{ }^{13} \mathrm{C}$ NMR $(125$ 
$\left.\mathrm{MHz}, \mathrm{MeOH}-\mathrm{d}_{4}\right) \delta 174.7,169.9,155.3,139.6,138.0,133.1,131.2,131.1,130.7,130.5,128.4,126.5$, 126.1, 117.1, 59.4, 55.8, 38.0, 31.6, 20.2, 19.5, 18.3; MS (ESI) m/z 357.2 [M+H] ${ }^{+}, 713.2[\mathrm{M}+\mathrm{Na}]^{+}$.

H-m-(o-tolyl)Tyr-Val-OH (10d). $97 \%$ purity; RP-HPLC $(10 \rightarrow 50 \%) t_{\mathrm{R}}=23.1 ;{ }^{1} \mathrm{H}$ NMR $(500 \mathrm{MHz}$, MeOH-d $\left.{ }_{4}\right) \delta 7.57-7.54(\mathrm{~m}, 2 \mathrm{H}), 7.40-7.36(\mathrm{~m}, 2 \mathrm{H}), 7.29(\mathrm{dd}, J=7.4,7.4 \mathrm{~Hz}, 1 \mathrm{H}), 7.20(\mathrm{~d}, J=2.2 \mathrm{~Hz}$, 1H), $7.10(\mathrm{dd}, J=8.3,2.3 \mathrm{~Hz}, 1 \mathrm{H}), 6.90(\mathrm{~d}, J=8.3 \mathrm{~Hz}, 1 \mathrm{H}), 4.26(\mathrm{~d}, J=5.2 \mathrm{~Hz}, 1 \mathrm{H}), 4.18(\mathrm{dd}, J=7.6,7.6$ Hz, 1H), $3.13(\mathrm{dd}, J=13.9,7.6 \mathrm{~Hz}, 1 \mathrm{H}), 3.04(\mathrm{dd}, J=13.9,7.5 \mathrm{~Hz}, 1 \mathrm{H}), 2.08(\mathrm{~m}, 1 \mathrm{H}), 0.82(\mathrm{~d}, J=6.9$ $\mathrm{Hz}, 3 \mathrm{H}), 0.79(\mathrm{~d}, J=6.9 \mathrm{~Hz}, 3 \mathrm{H}) ;{ }^{13} \mathrm{C} \mathrm{NMR}\left(125 \mathrm{MHz}, \mathrm{MeOH}-\mathrm{d}_{4}\right) \delta 174.9,169.9,155.2,139.8,132.8$, $130.6,130.5,130.4,129.0,127.9,126.5,117.6,59.5,55.9,38.1,31.6,19.5,18.2 ; \mathrm{MS}(\mathrm{ESI}) \mathrm{m} / \mathrm{z} 371.2$ $[\mathrm{M}+\mathrm{H}]^{+}, 741.2[\mathrm{M}+\mathrm{Na}]^{+}$.

H-1-(4-hydroxy)Nal-Val-OH (16). 98\% purity; RP-HPLC $(10 \rightarrow 80 \%) t_{\mathrm{R}}=14.7 ;{ }^{1} \mathrm{H}$ NMR $(500$ $\left.\mathrm{MHz}, \mathrm{MeOH}-\mathrm{d}_{4}\right) \delta 8.29(\mathrm{~d}, J=8.4 \mathrm{~Hz}, 1 \mathrm{H}), 8.11(\mathrm{~d}, J=8.5 \mathrm{~Hz}, 1 \mathrm{H}), 7.57(\mathrm{dd}, J=7.6,7.6 \mathrm{~Hz}, 1 \mathrm{H}), 7.47$ $(\mathrm{dd}, J=7.6,7.6 \mathrm{~Hz}, 1 \mathrm{H}), 7.26(\mathrm{~d}, J=7.7 \mathrm{~Hz}, 1 \mathrm{H}), 6.80(\mathrm{~d}, J=7.70 \mathrm{~Hz}, 1 \mathrm{H}), 4.32$ (d, J=5.6 Hz, 1H), 4.28 (dd, $J=8.2,6.6 \mathrm{~Hz}, 1 \mathrm{H}), 3.64(\mathrm{dd}, J=14.6,6.5 \mathrm{~Hz}, 1 \mathrm{H}), 3.37(\mathrm{dd}, J=14.6,8.3 \mathrm{~Hz}, 1 \mathrm{H}), 2.17(\mathrm{~m}, 1 \mathrm{H})$, $0.98(\mathrm{~d}, J=6.9 \mathrm{~Hz}, 6 \mathrm{H}) ;{ }^{13} \mathrm{C} \mathrm{NMR}\left(125 \mathrm{MHz}, \mathrm{MeOH}-\mathrm{d}_{4}\right) \delta 173.8,170.1,155.0,134.4,130.1,127.9$, $127.1,125.7,124.3,124.1,121.5,108.5,59.5,54.8,35.4,31.8,19.4,18.5 ; \mathrm{MS}(\mathrm{ESI}) \mathrm{m} / \mathrm{z} 331.1$ $[\mathrm{M}+\mathrm{H}]^{+}$.

H-(D/L)-1-(4-hydroxy)Nal-Val-OH (17). RP-HPLC $(10 \rightarrow 80 \%) t_{\mathrm{R}}=14.6,18.5 ;{ }^{1} \mathrm{H}$ NMR $(500 \mathrm{MHz}$, MeOH-d $\left.{ }_{4}\right) \delta 8.28(\mathrm{dd}, J=8.0,5.4 \mathrm{~Hz}, 2 \mathrm{H}), 8.10(\mathrm{~d}, J=8.5 \mathrm{~Hz}, 1 \mathrm{H}), 8.04(\mathrm{~d}, J=8.5 \mathrm{~Hz}, 1 \mathrm{H}), 7.57(\mathrm{dd}$, $J=7.7,7.7 \mathrm{~Hz}, 2 \mathrm{H}), 7.47(\mathrm{dd}, J=7.7,7.7 \mathrm{~Hz}, 2 \mathrm{H}), 7.26(\mathrm{~d}, J=7.7 \mathrm{~Hz}, 1 \mathrm{H}), 7.22(\mathrm{~d}, J=7.7 \mathrm{~Hz}, 1 \mathrm{H}), 6.80$ (d, J=7.4 Hz, 1H), $6.79(\mathrm{~d}, J=7.5 \mathrm{~Hz}, 1 \mathrm{H}), 4.34-4.23(\mathrm{~m}, 3 \mathrm{H}), 4.07(\mathrm{~d}, J=5.6 \mathrm{~Hz}, 1 \mathrm{H}), 3.64$ (dd, $J=14.6,6.5 \mathrm{~Hz}, 1 \mathrm{H}), 3.54(\mathrm{dd}, J=13.9,9.2 \mathrm{~Hz}, 1 \mathrm{H}), 3.44(\mathrm{dd}, J=13.9,6.6 \mathrm{~Hz}, 1 \mathrm{H}), 3.37$ (dd, $J=14.6$, $8.3 \mathrm{~Hz}, 1 \mathrm{H}), 2.22-2.11(\mathrm{~m}, 1 \mathrm{H}), 1.88-1.78(\mathrm{~m}, 1 \mathrm{H}), 0.98(\mathrm{~d}, J=6.9 \mathrm{~Hz}, 6 \mathrm{H}), 0.62(\mathrm{~d}, J=6.9 \mathrm{~Hz}, 3 \mathrm{H})$, $0.59(\mathrm{~d}, J=6.9 \mathrm{~Hz}, 3 \mathrm{H})$. 
NMR spectra of (S)-- $N^{\alpha}$-tert-butyloxycarbonyl-m-iodotyrosine (5).
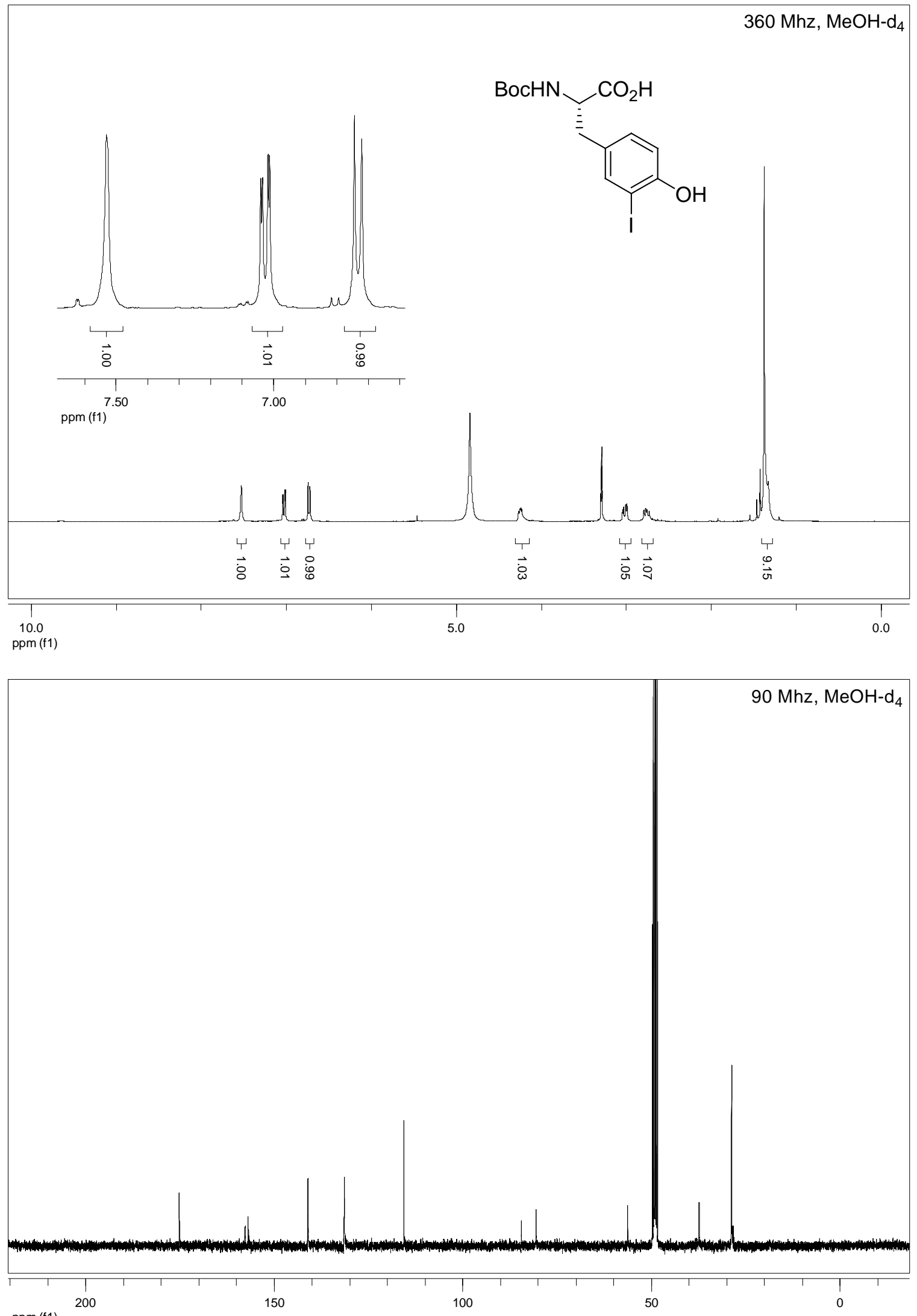

ppm (f1) 
NMR spectra of $(S)$ - $N^{\alpha}$-tert-butyloxycarbonyl-O-benzyl-m-iodotyrosine benzyl ester (6).

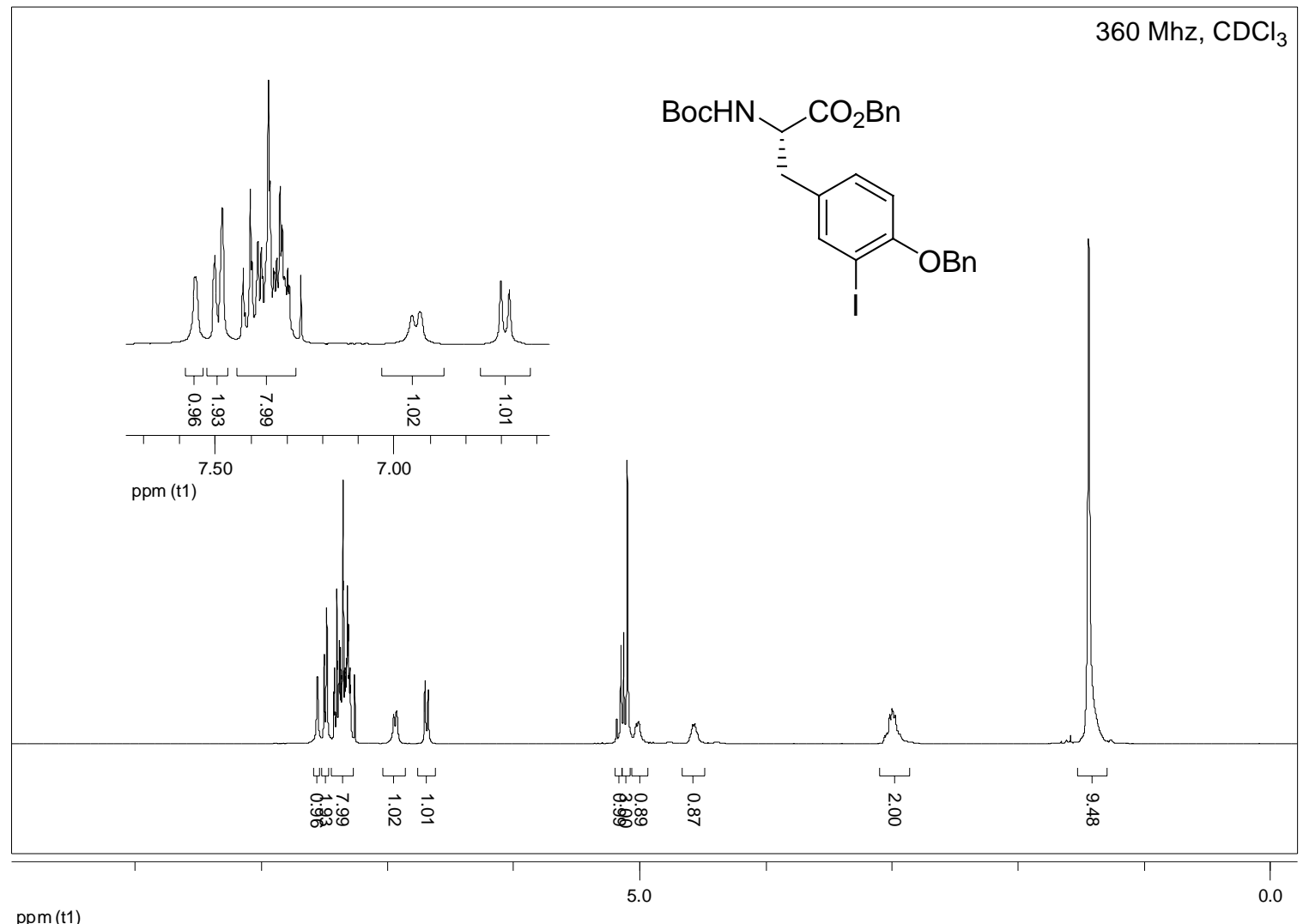

ppm (t1)

$90 \mathrm{Mhz}, \mathrm{CDCl}_{3}$

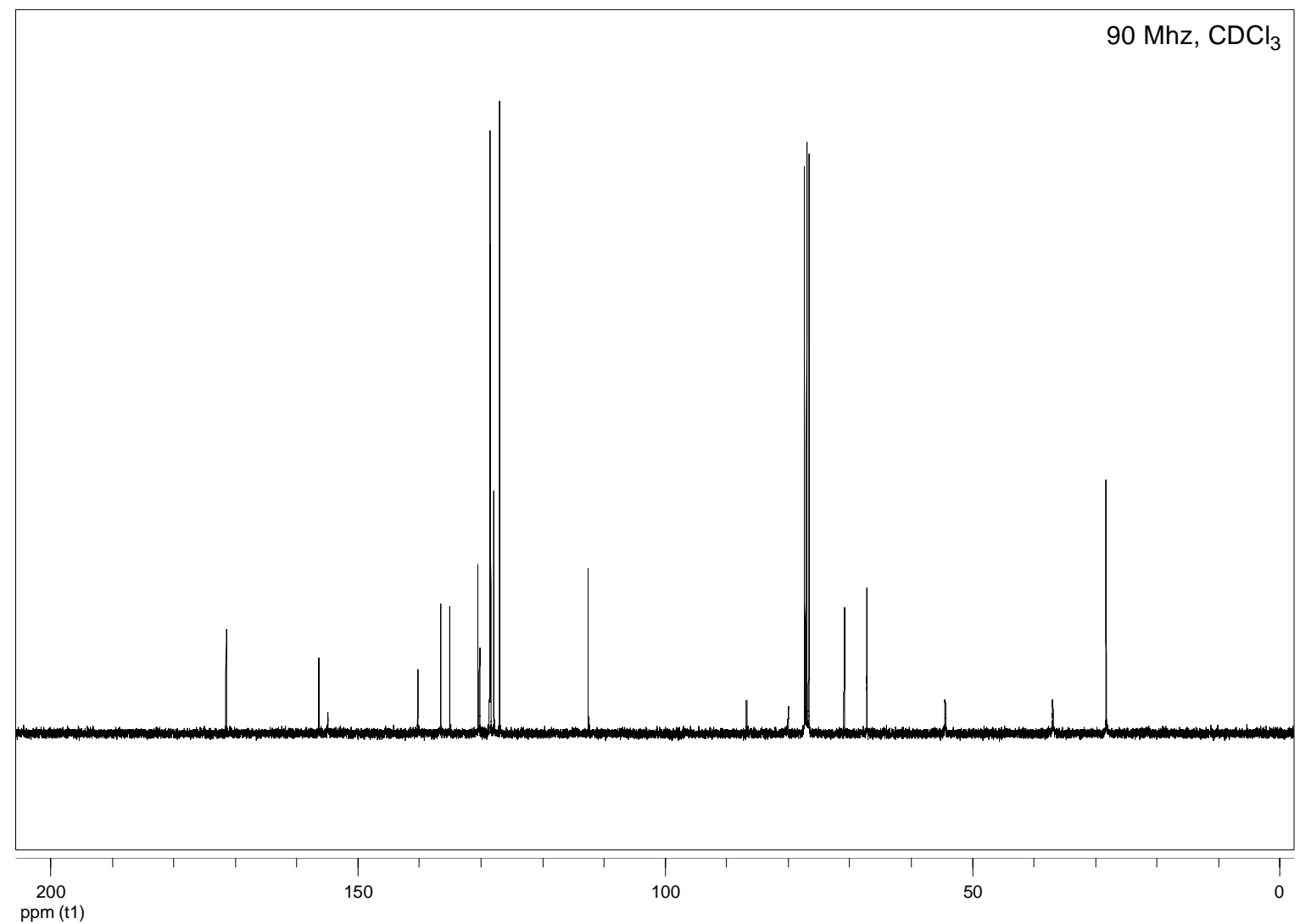


NMR spectra of $(S)$ - $N^{\alpha}$-tert-butyloxycarbonyl-O-benzyl- $m$-phenyltyrosine benzyl ester (7a).
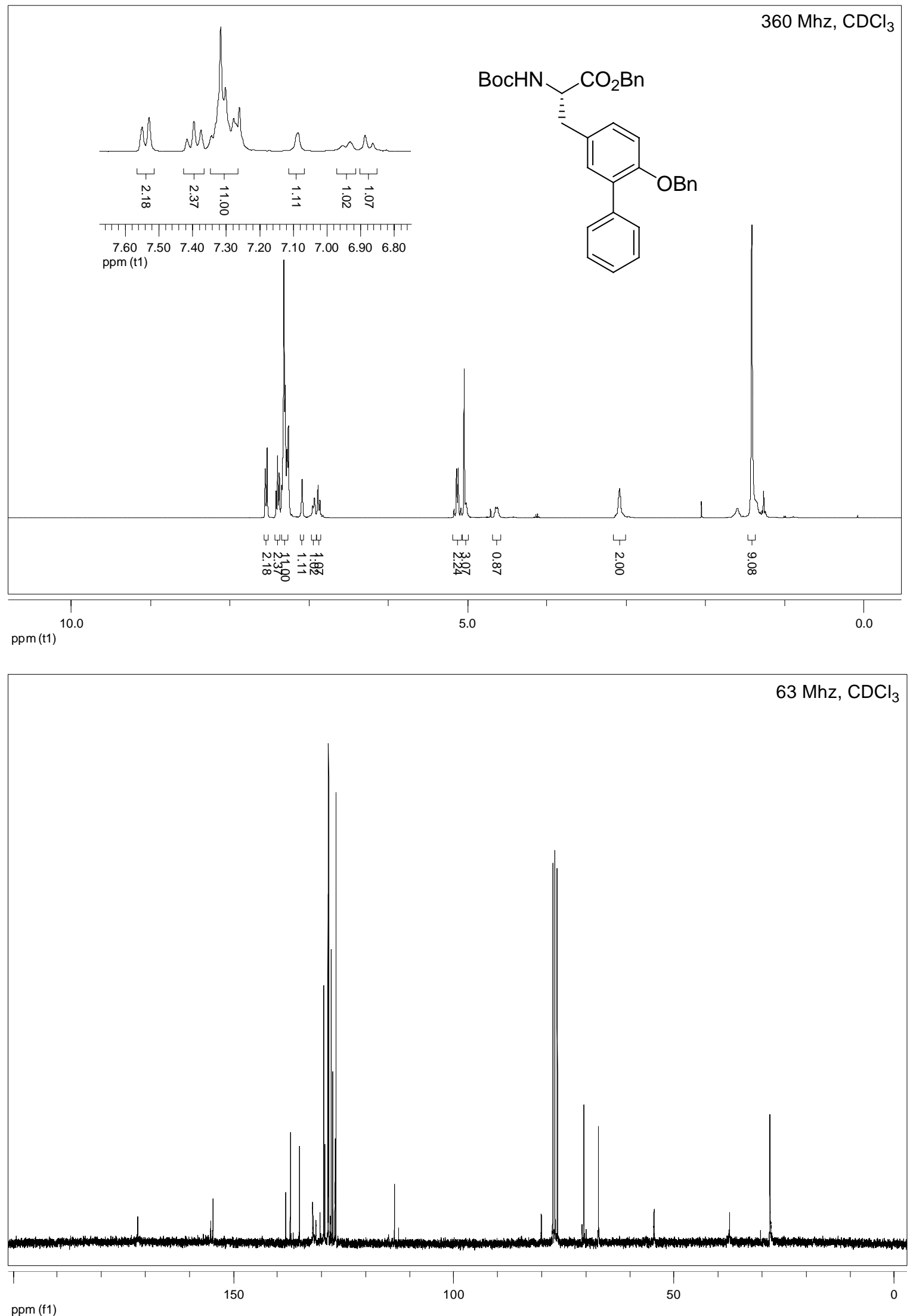
NMR spectra of $(S)$ - $N^{\alpha}$-tert-butyloxycarbonyl- $O$-benzyl- $m$-( $p$-tolyl)tyrosine benzyl ester (7b).
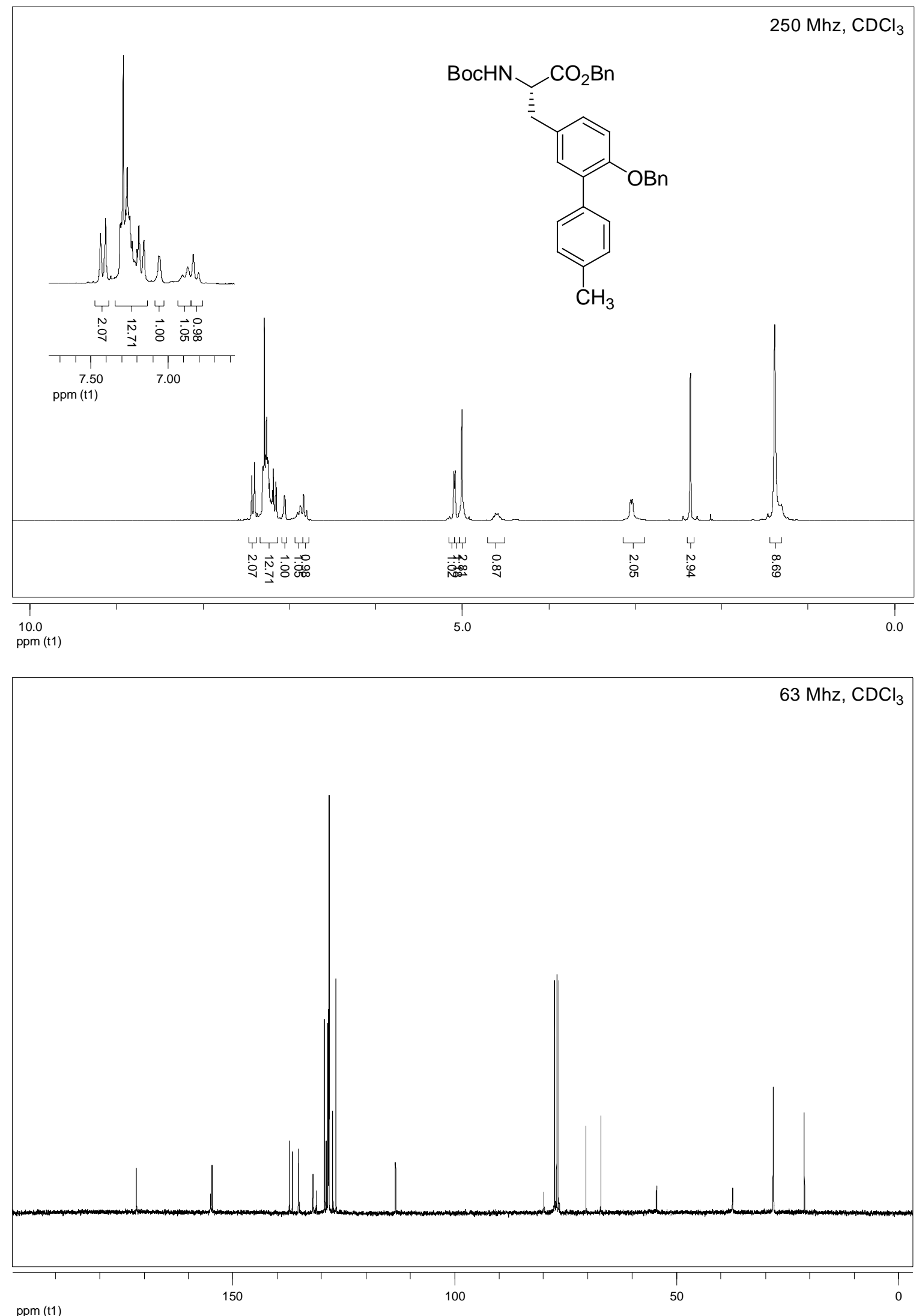
NMR spectra of $(S)-N^{\alpha}$-tert-butyloxycarbonyl-O-benzyl-m-(m-tolyl)tyrosine benzyl ester (7c).
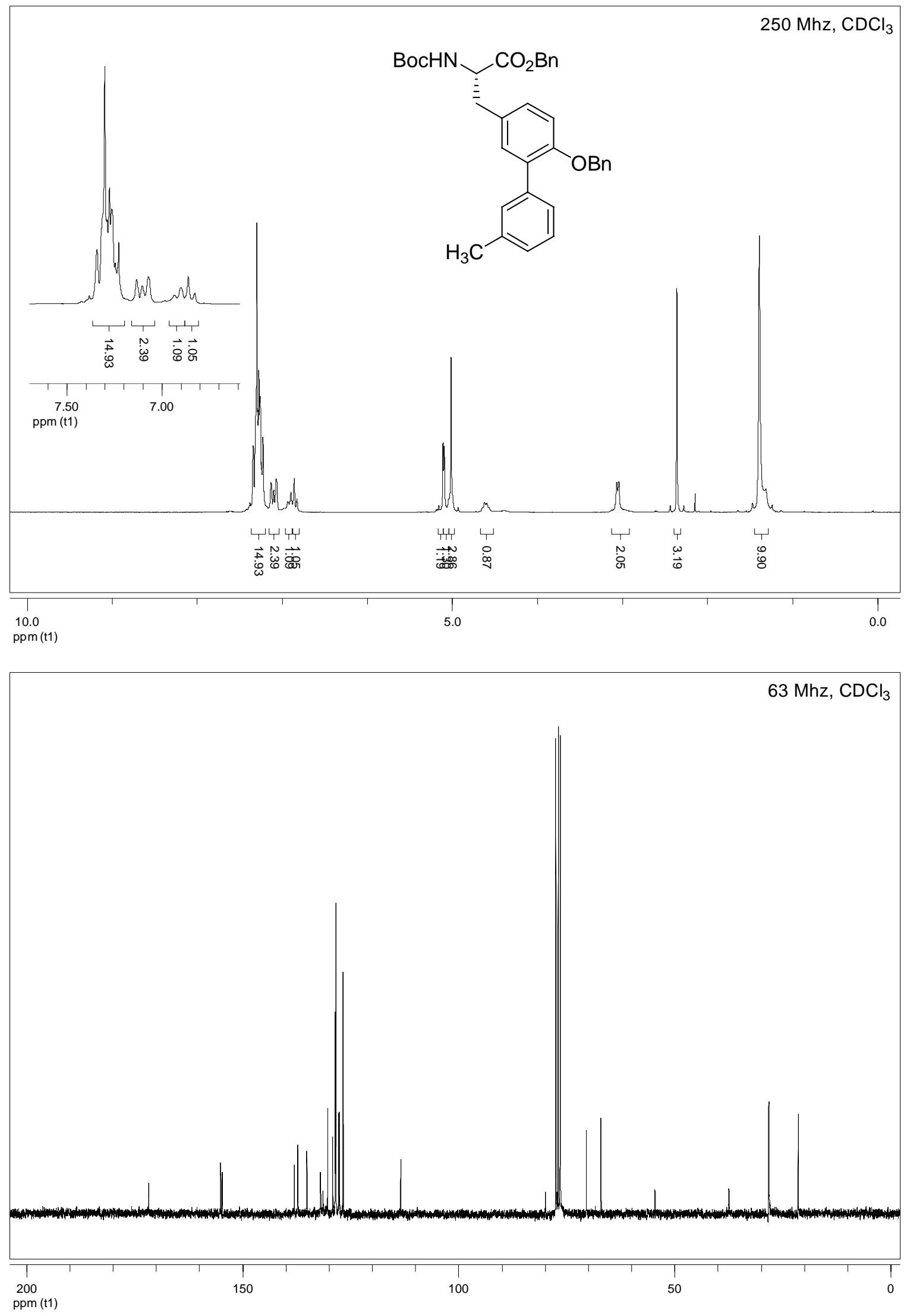
NMR spectra of $(S)$ - $N^{\alpha}$-tert-butyloxycarbonyl-O-benzyl-m-(o-tolyl)tyrosine benzyl ester (7d).
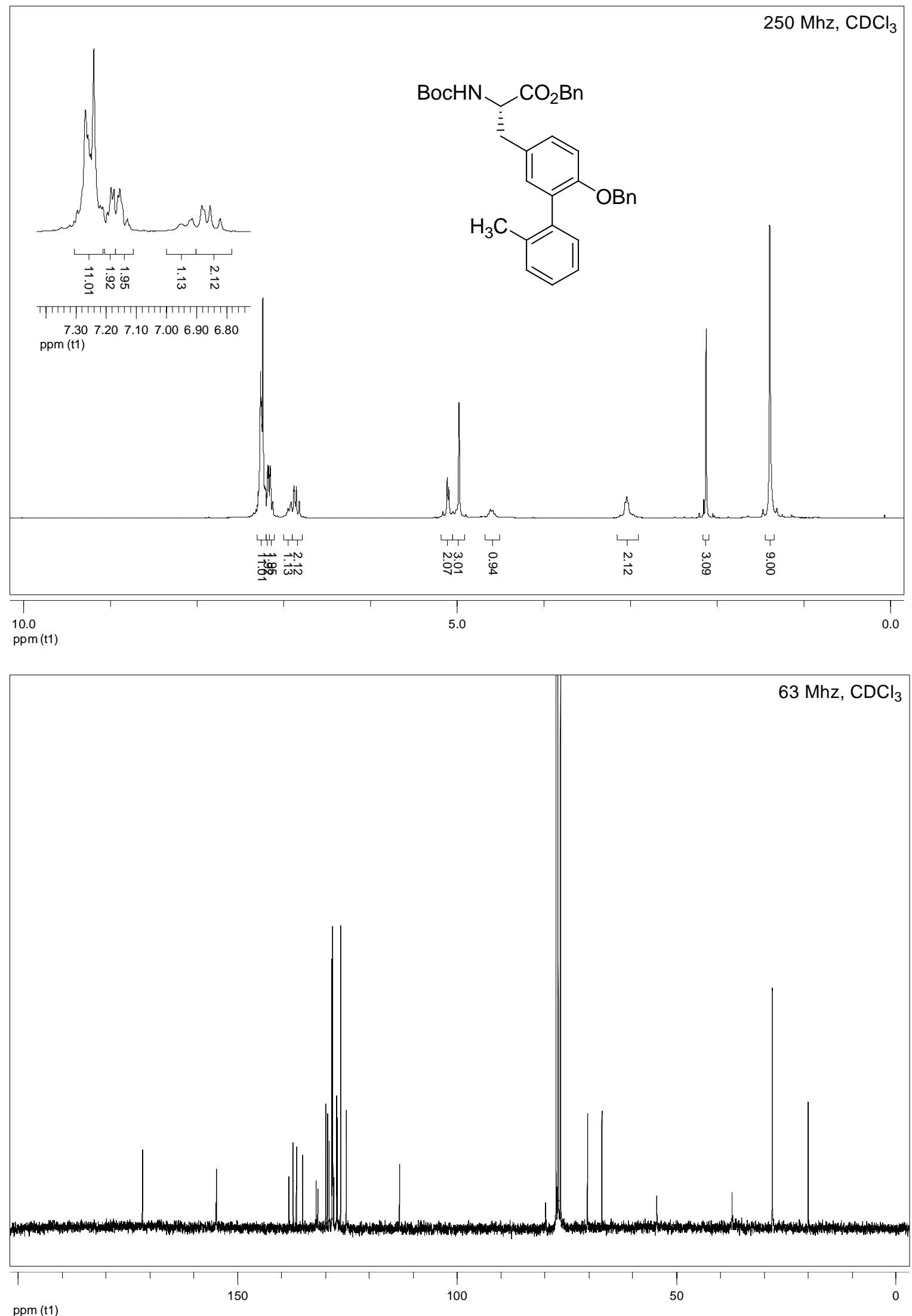
NMR spectra of $(S)$ - $N^{\alpha}$-tert-butyloxycarbonyl-O-benzyl-m-(3-hydroxyphenyl)tyrosine benzyl ester (7e).
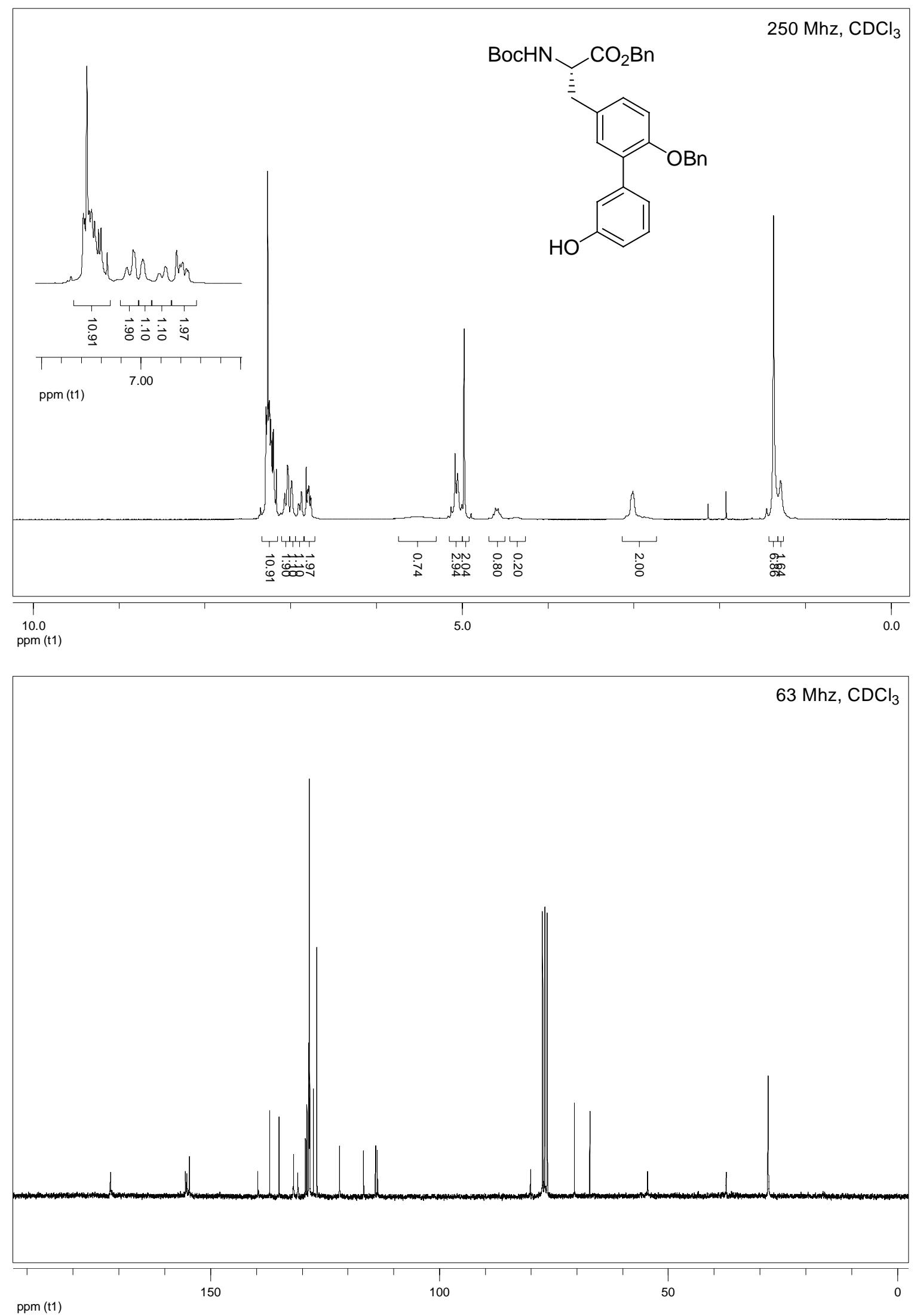
NMR spectra of $(S)$ - $N^{\alpha}$-tert-butyloxycarbonyl- $O$-benzyl-m-(3-chlorophenyl)tyrosine benzyl ester (7f).

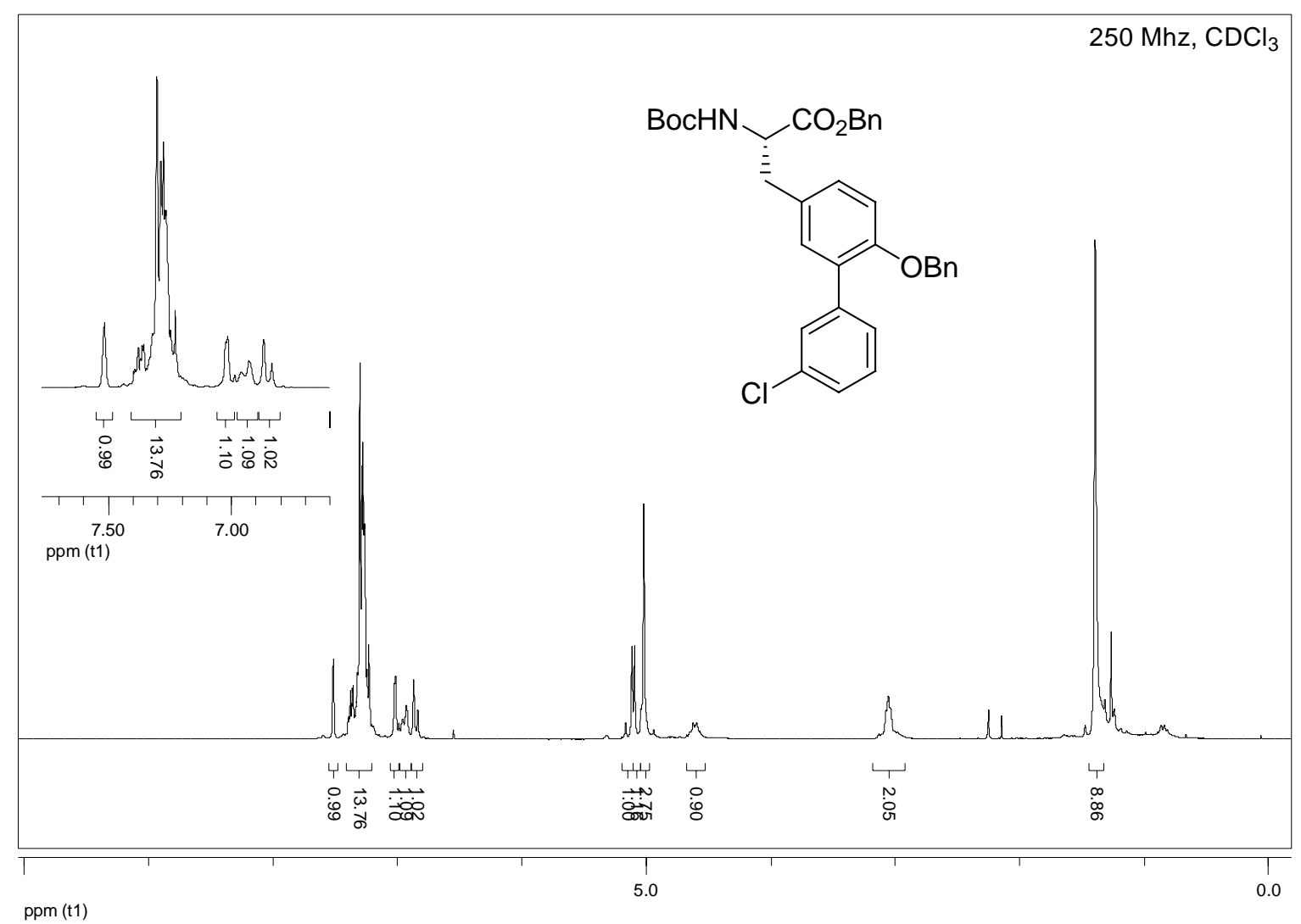

$63 \mathrm{Mhz} \mathrm{CDCl}_{3}$

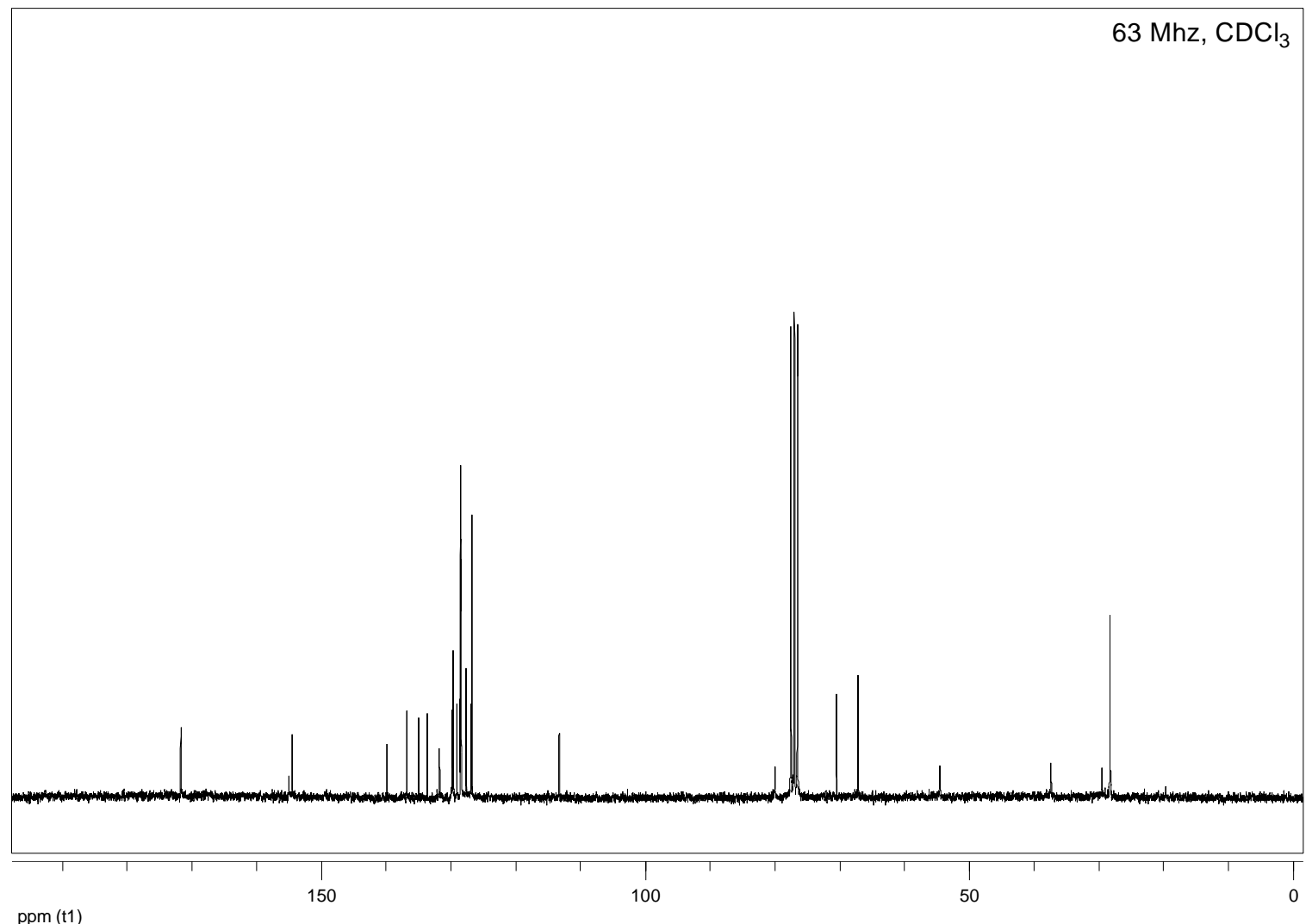


NMR spectra of $(S)$ - $N^{\alpha}$-tert-butyloxycarbonyl-O-benzyl-m-(4-fluorophenyl)tyrosine benzyl ester (7g).

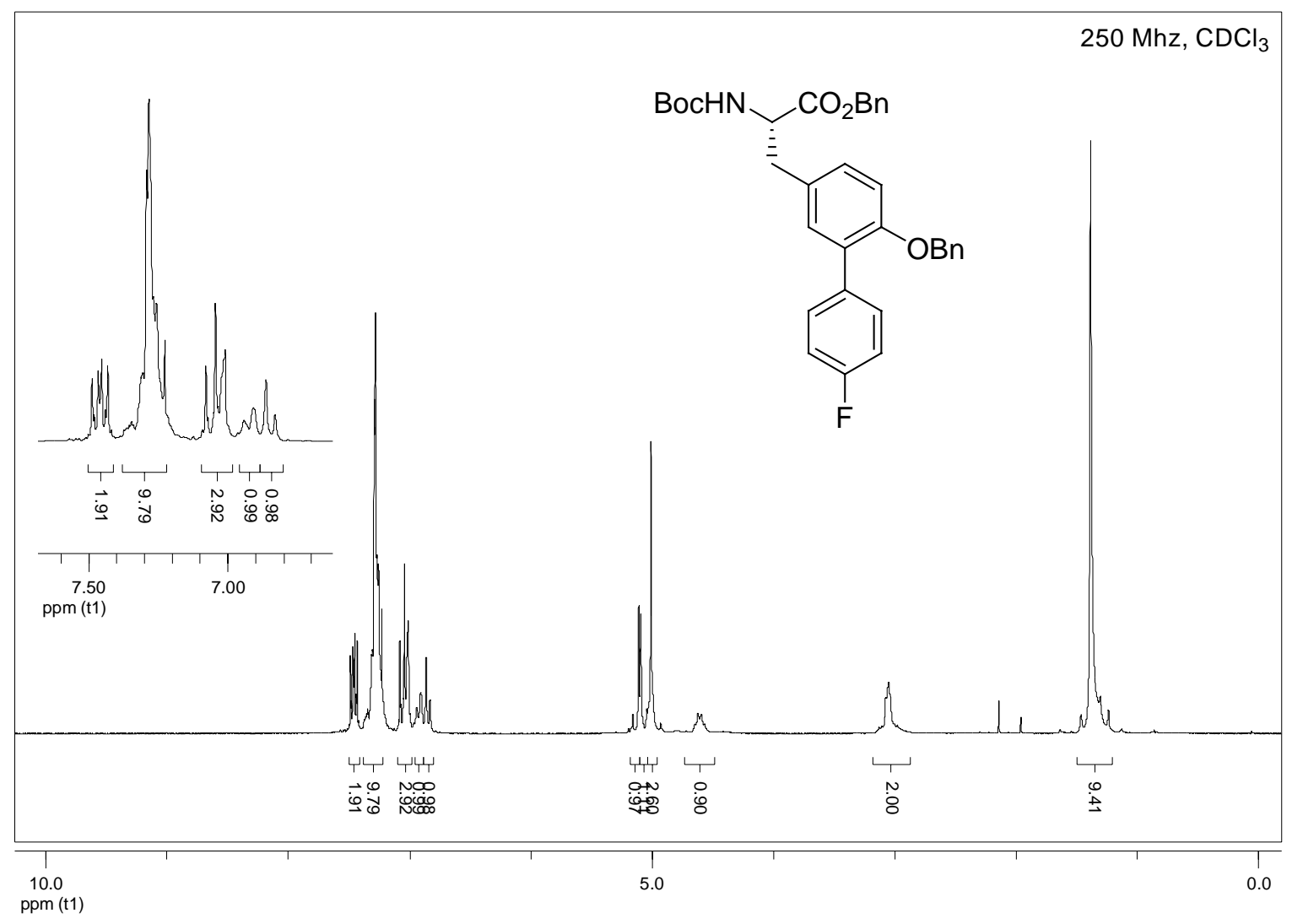

$63 \mathrm{Mhz}, \mathrm{CDCl}_{3}$

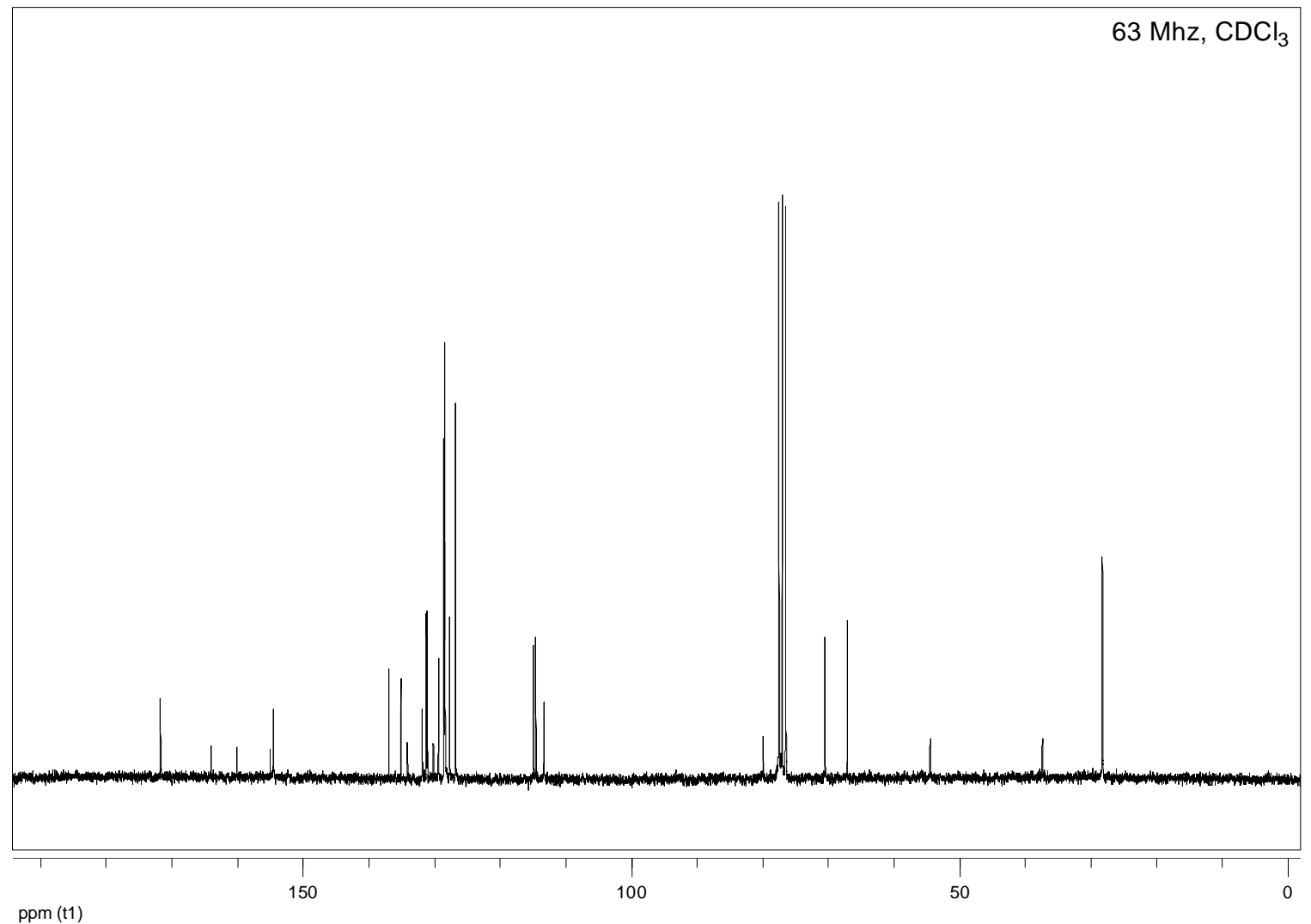


NMR spectra of $(S)$ - $N^{\alpha}$-tert-butyloxycarbonyl- $O$-benzyl-m-(4-carboxyphenyl)tyrosine benzyl ester (7h).
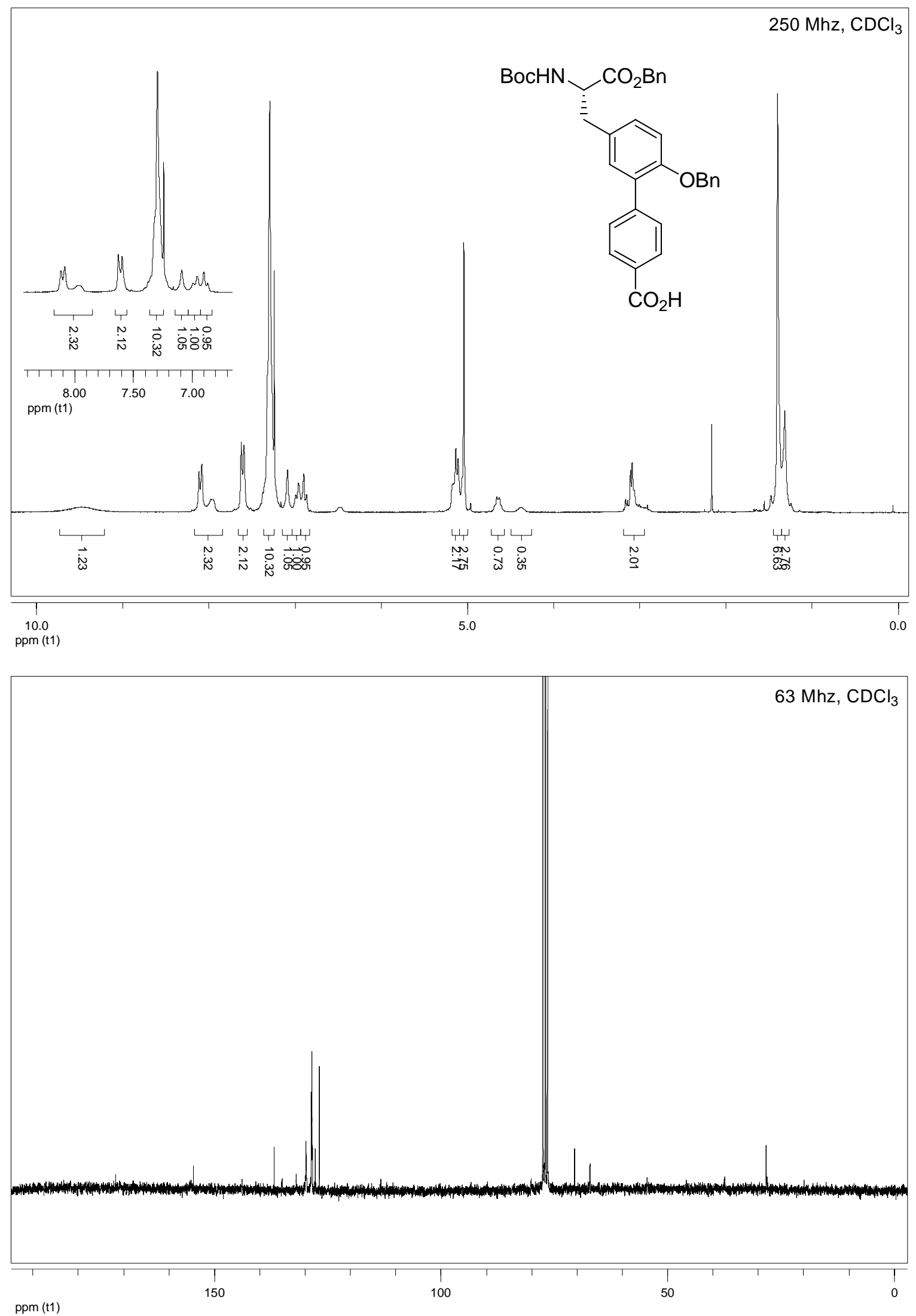

ppm (t1) 
NMR spectra of $(S)$ - $N^{\alpha}$-tert-butyloxycarbonyl-O-benzyl- $m$-(2-furanyl)tyrosine benzyl ester (7i).
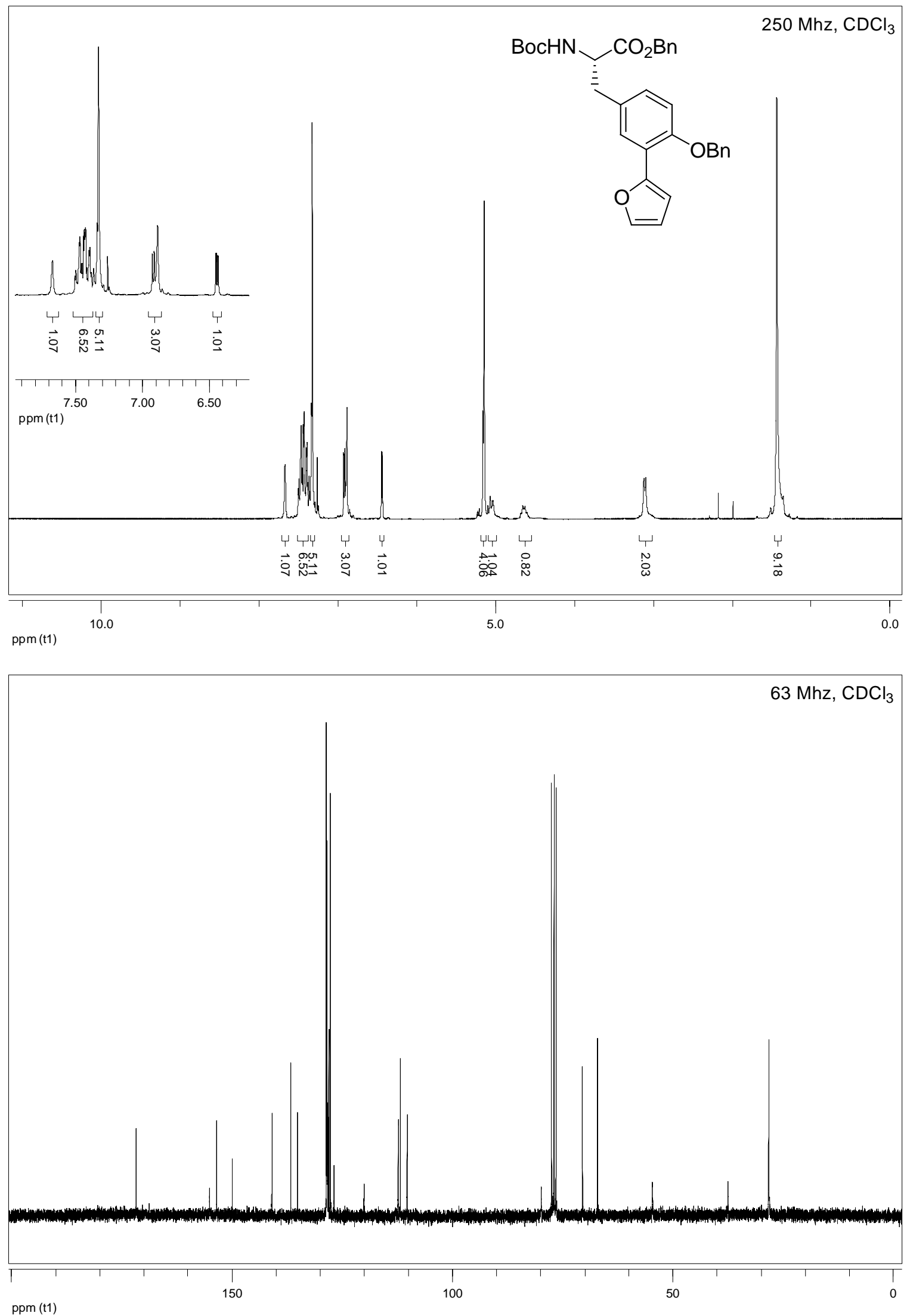
NMR spectra of $(S)$ - $N^{a}$-tert-butyloxycarbonyl-O-benzyl- $m$-(3-thienyl)tyrosine benzyl ester (7j).
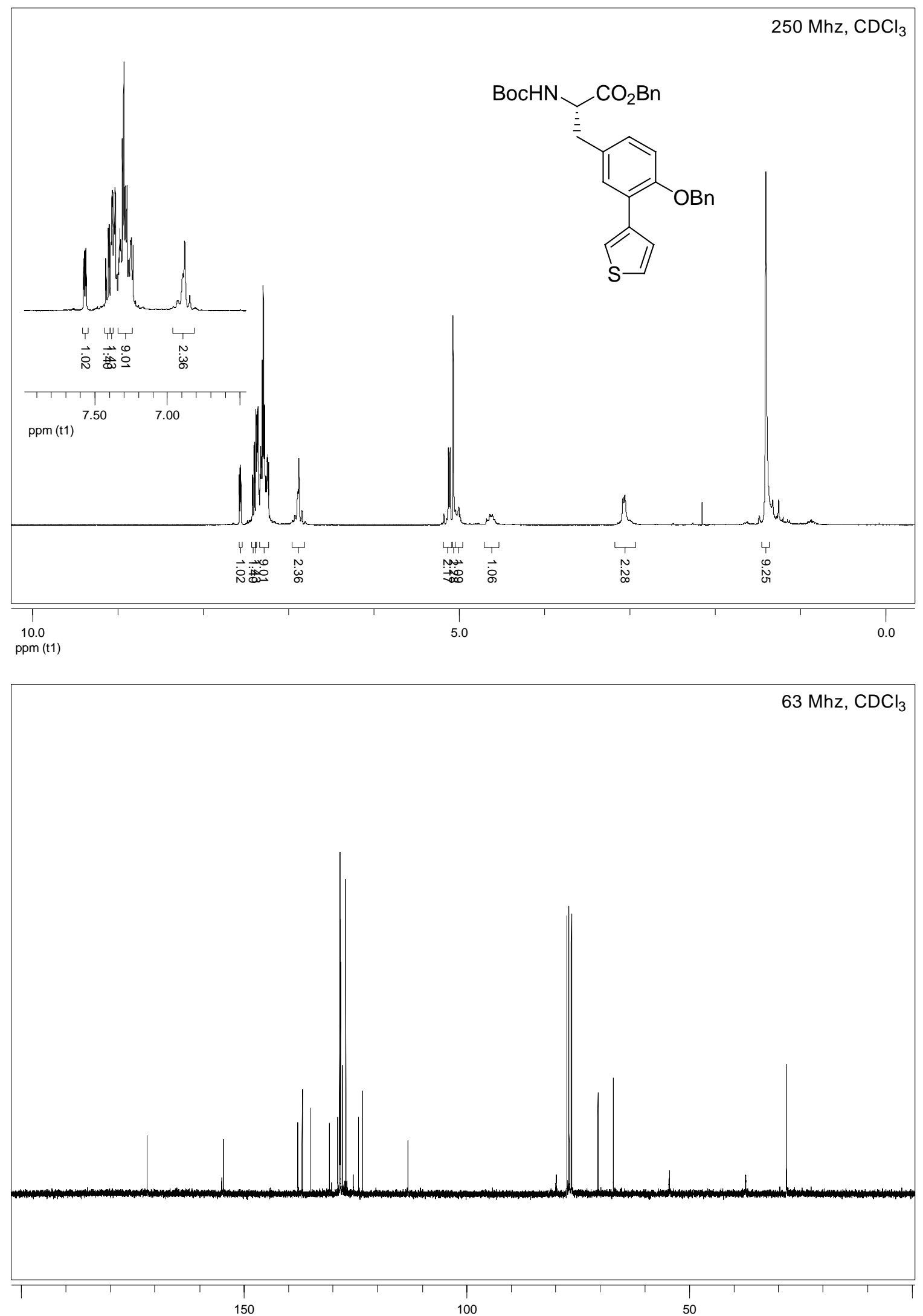

ppm (t1) 
NMR spectra of $(S)$ - $N^{\alpha}$-tert-butyloxycarbonyl-O-benzyl- $m$-phenyltyrosine (8a).
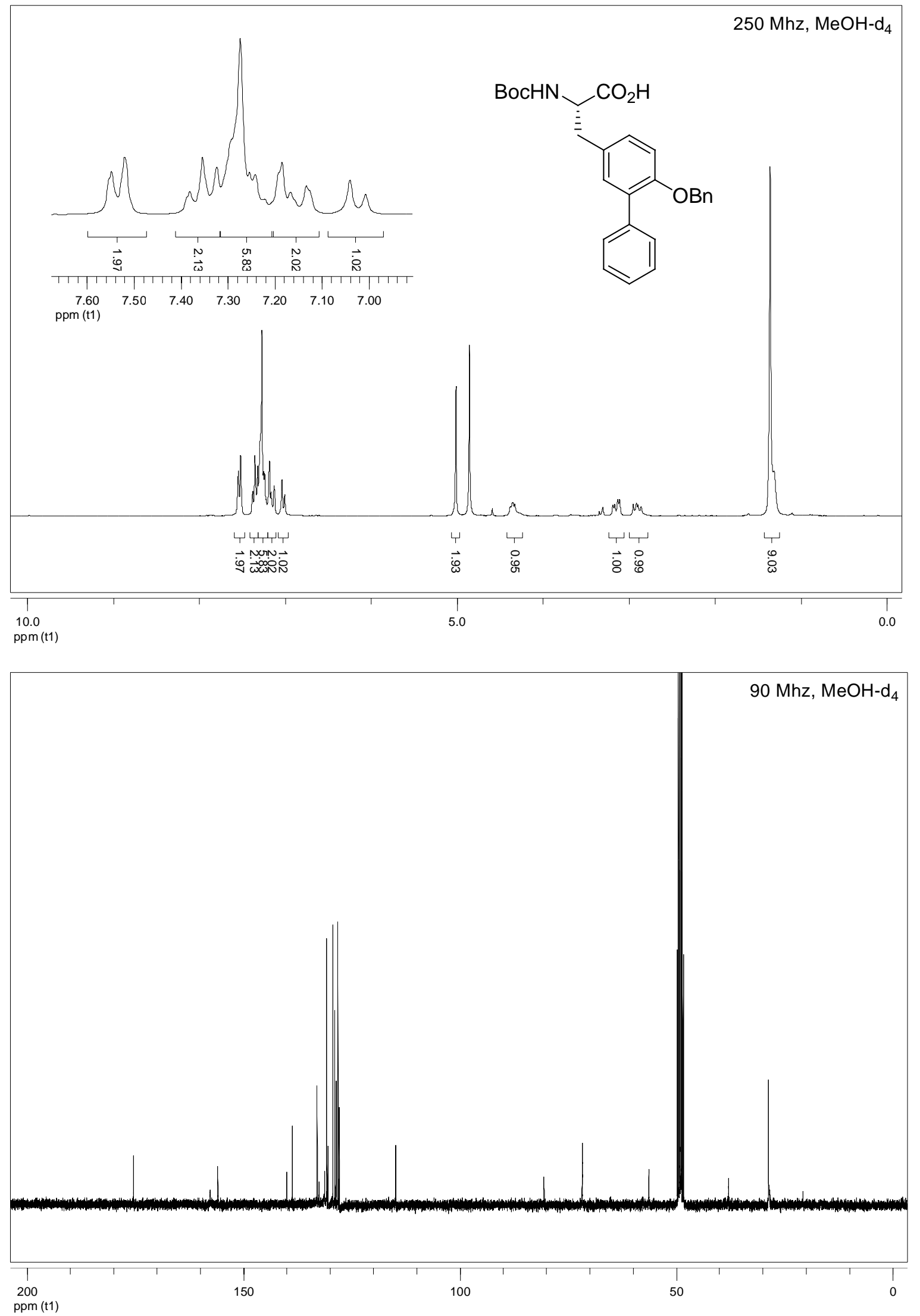
NMR spectra of $(S)$ - $N^{\alpha}$-tert-butyloxycarbonyl- $O$-benzyl-m-(o-tolyl)tyrosine $(\mathbf{8 b})$.
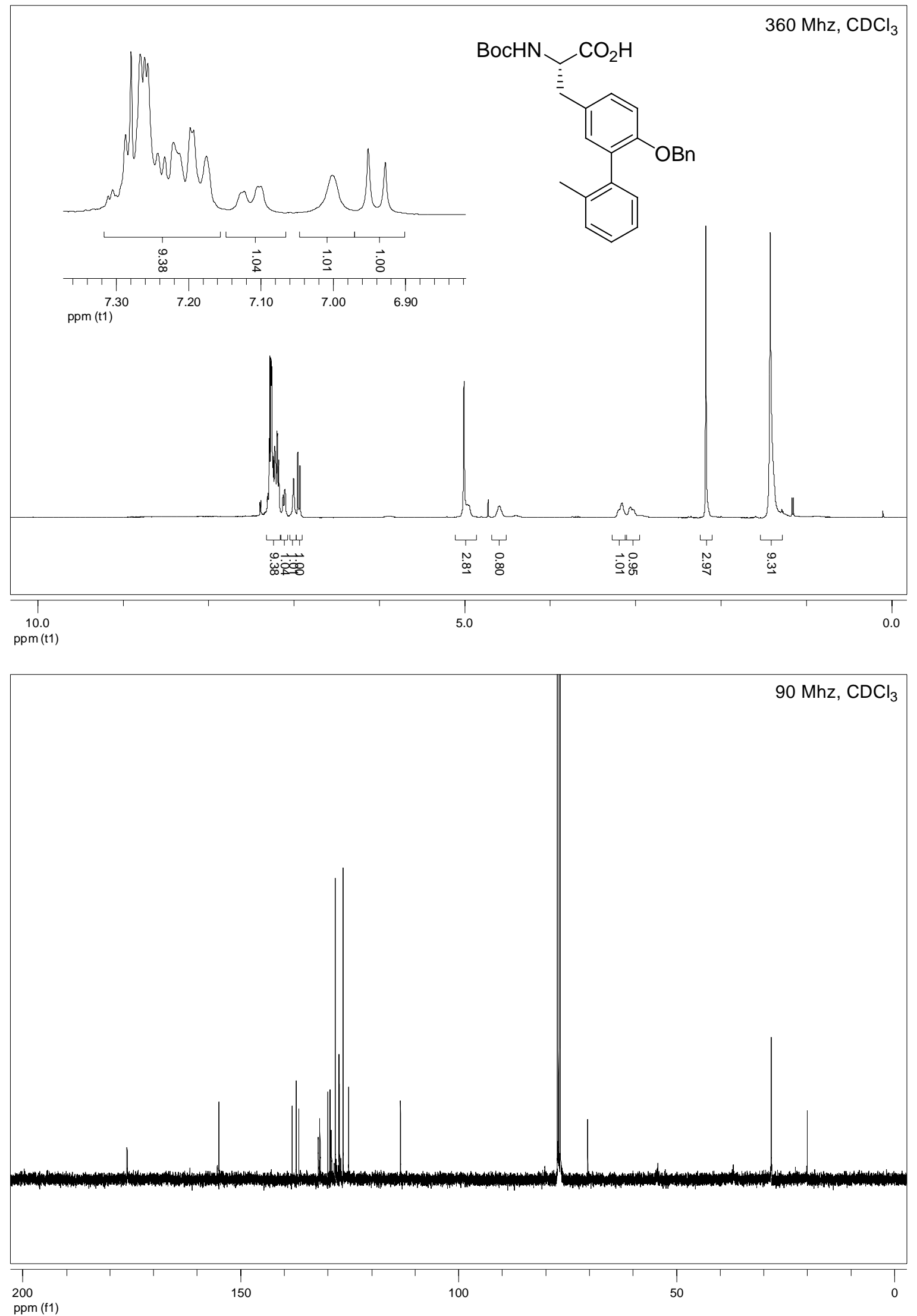
NMR spectra of $(S)$ - $N^{\alpha}$-tert-butyloxycarbonyl-m-phenyltyrosine (9a).
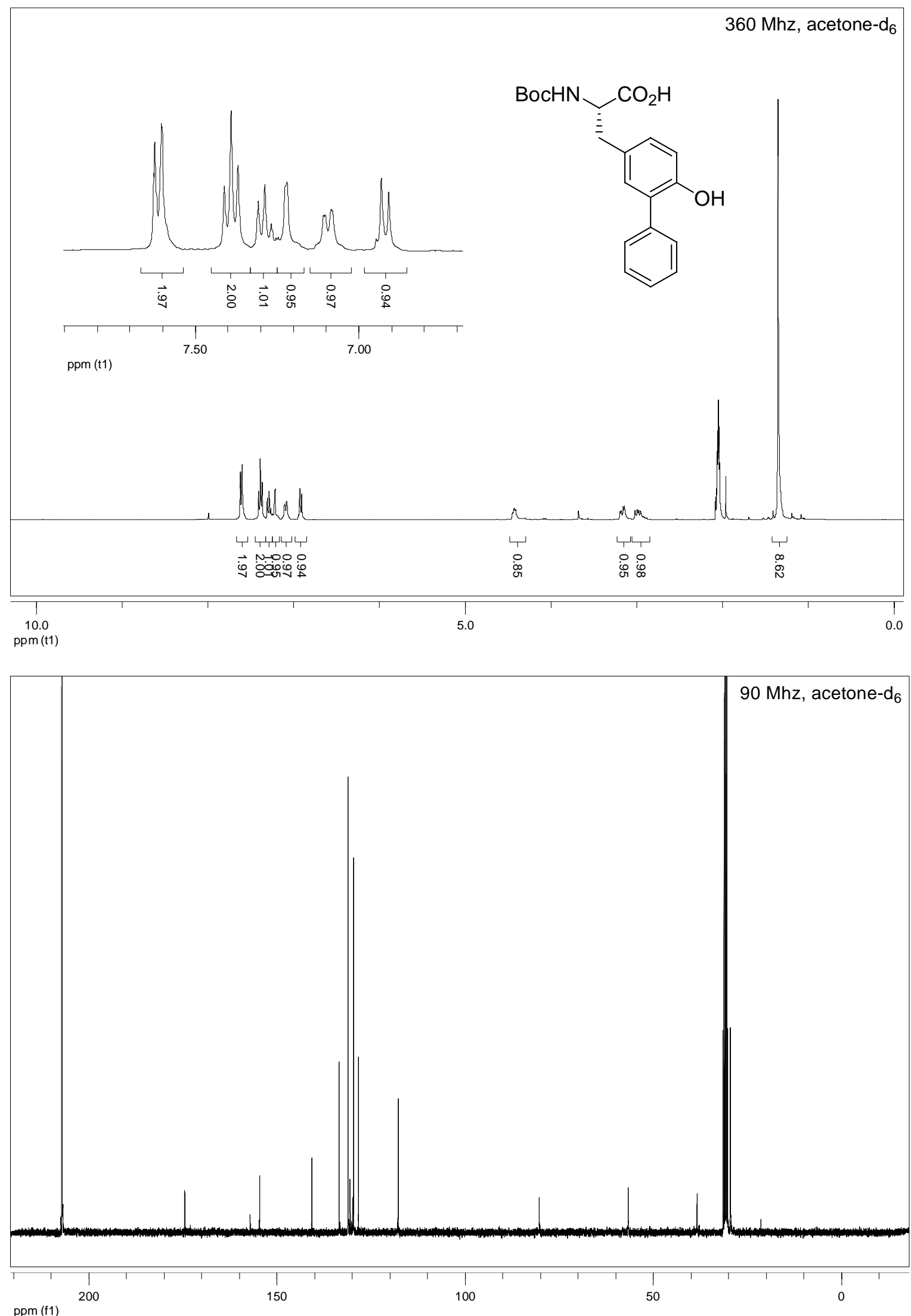
NMR spectra of $(S)-N^{\alpha}$-tert-butyloxycarbonyl-m-(o-tolyl)tyrosine (9b).
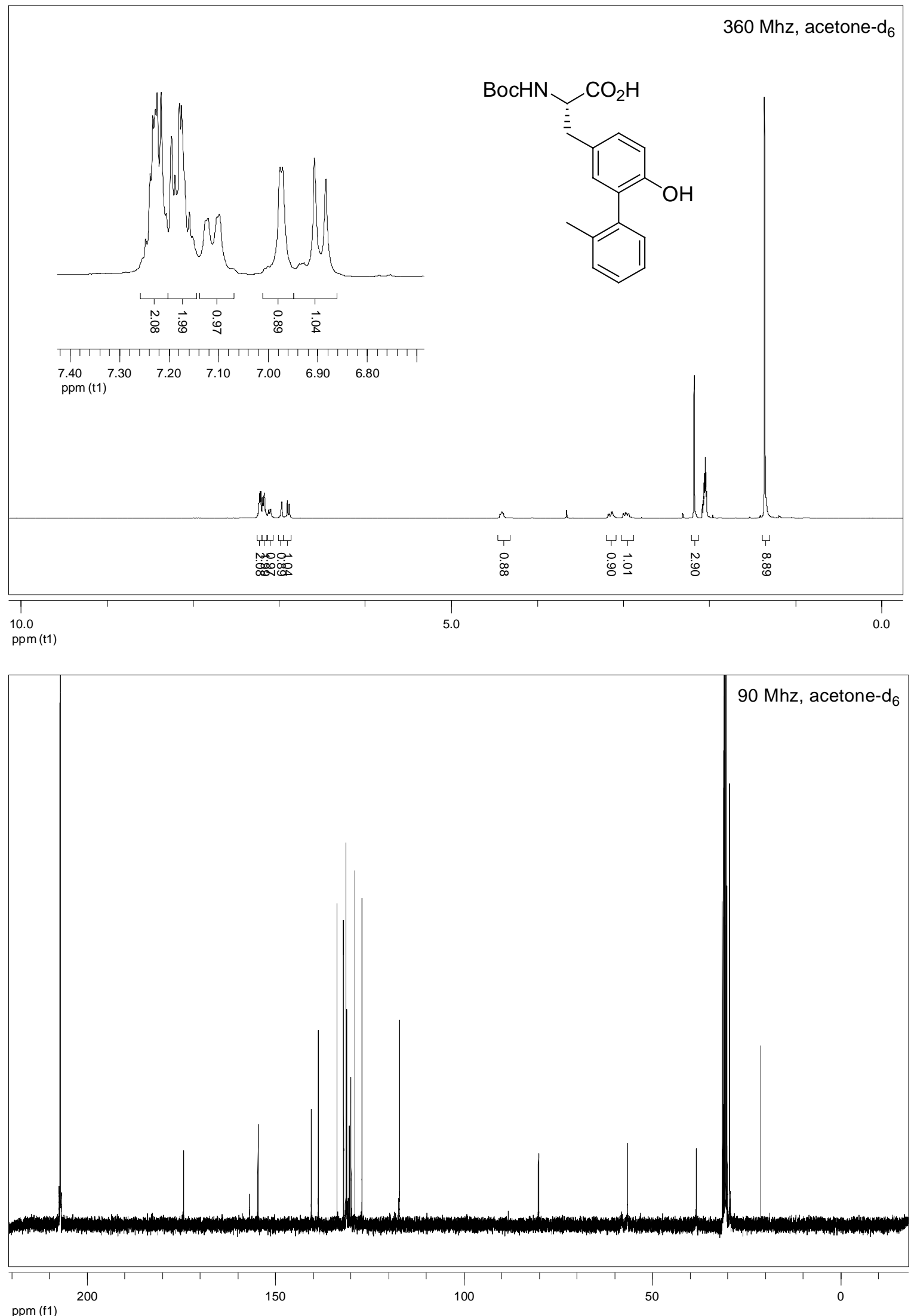
NMR spectra of 4-(tert-butyldimethylsilyloxy)naphthalene-1-carbaldehyde (12).

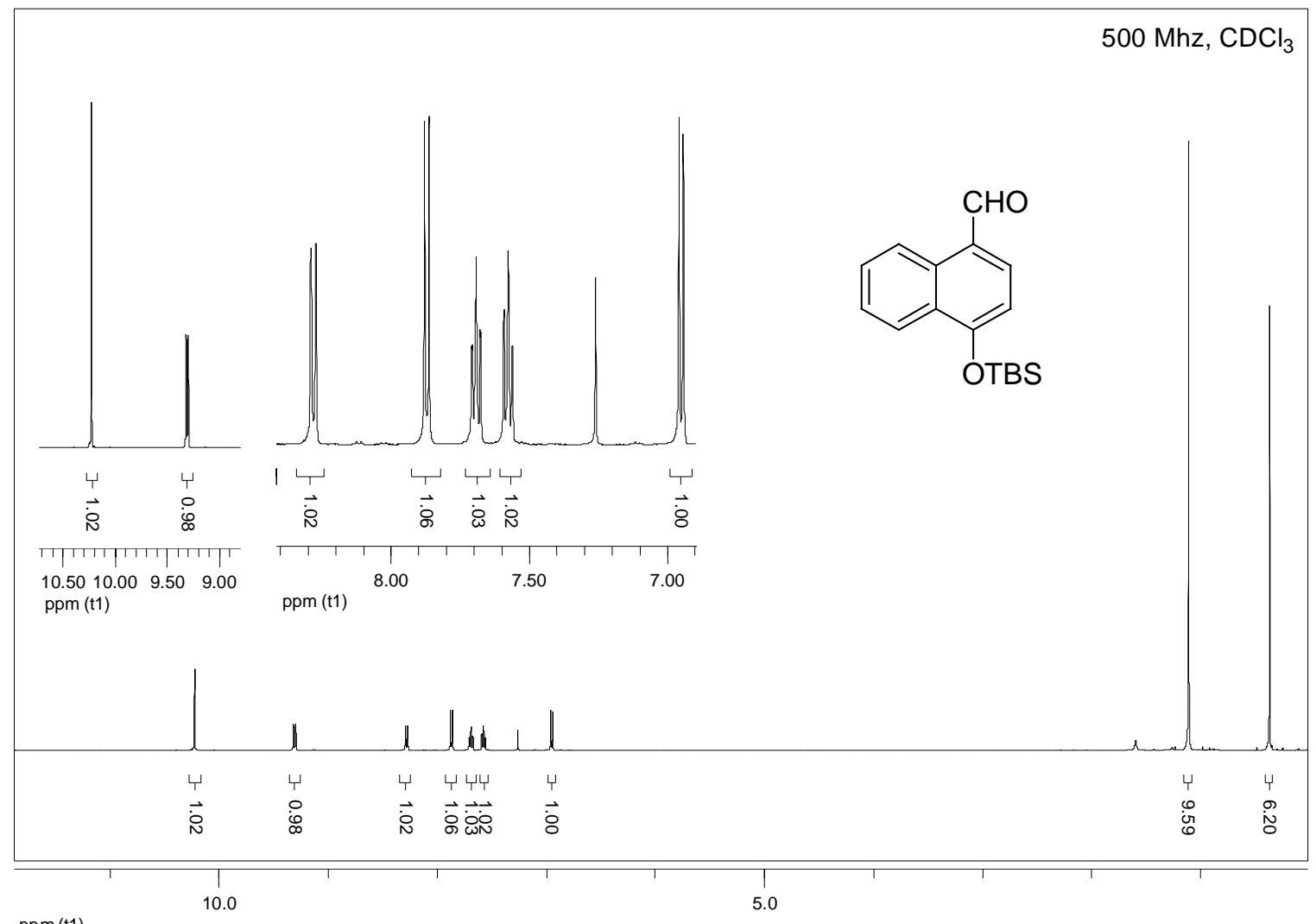

ppm (t1)

$63 \mathrm{Mhz}, \mathrm{CDCl}_{3}$

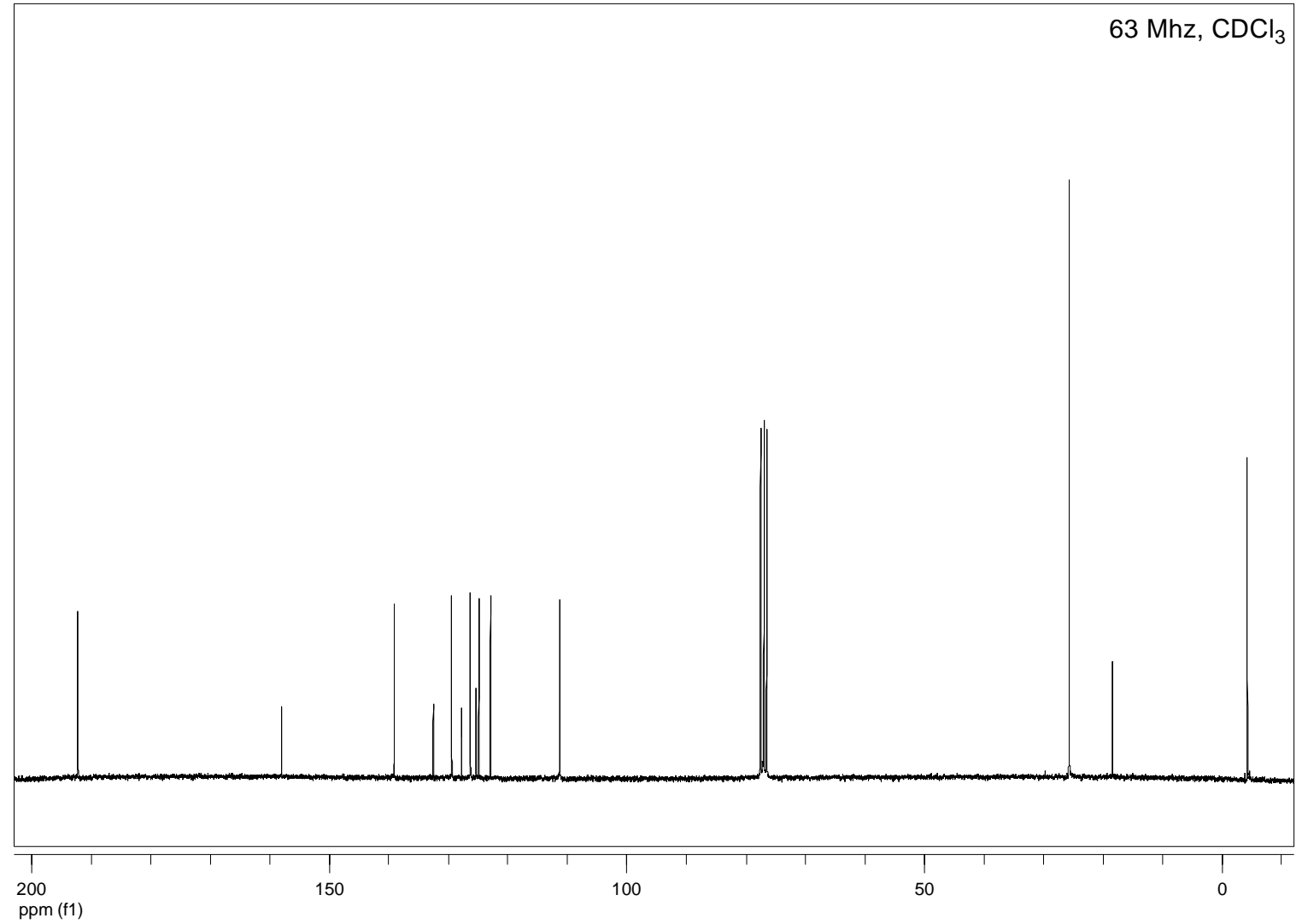


NMR spectra of (Z)-methyl 2-(tert-butyloxycarbonyl)amino-3-(1-(tert-butyldimethylsilyloxy) naphthalene-4-yl) acrylate (13).

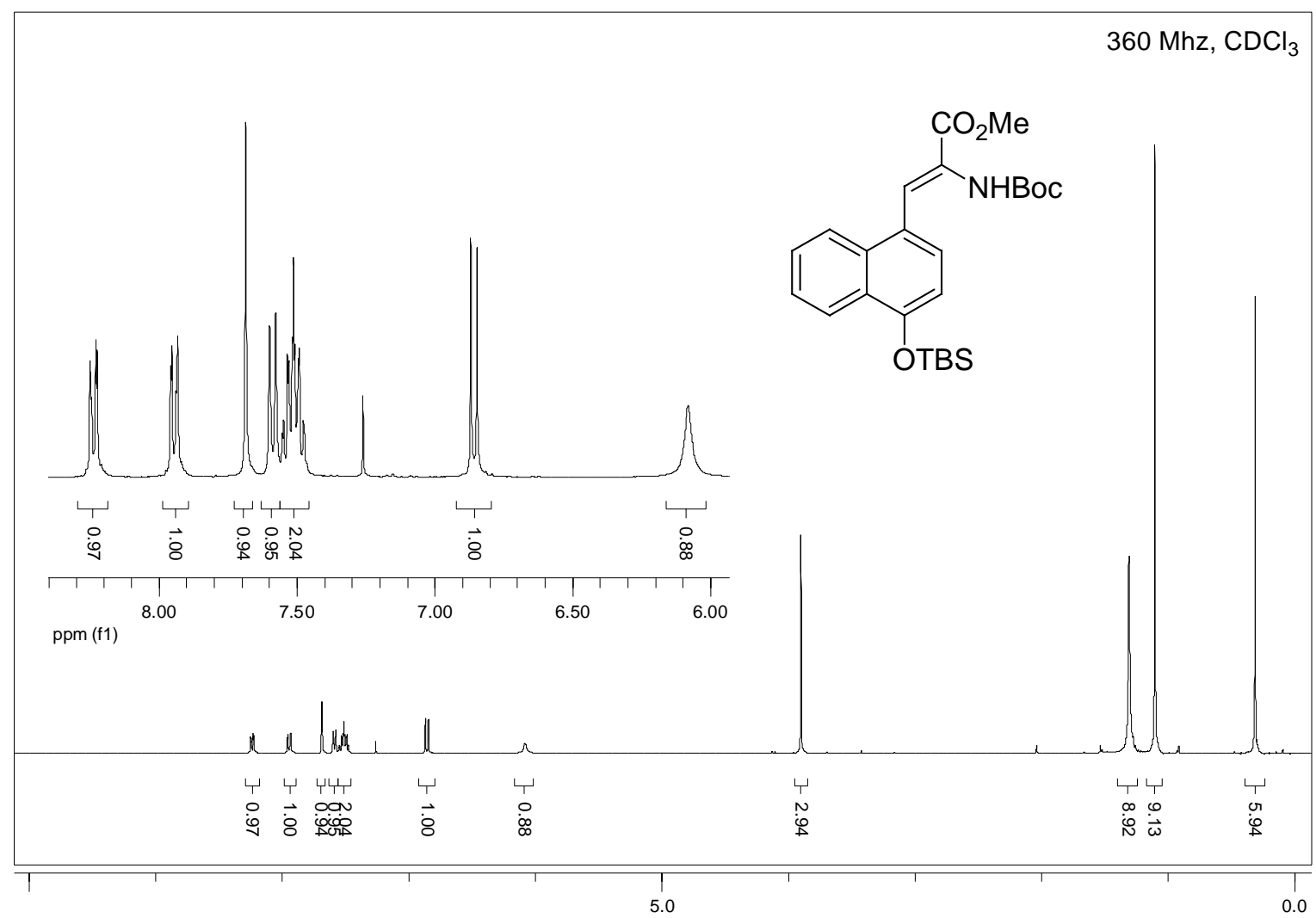

$\operatorname{ppm}(\mathrm{f} 1)$

$63 \mathrm{Mhz}, \mathrm{CDCl}_{3}$

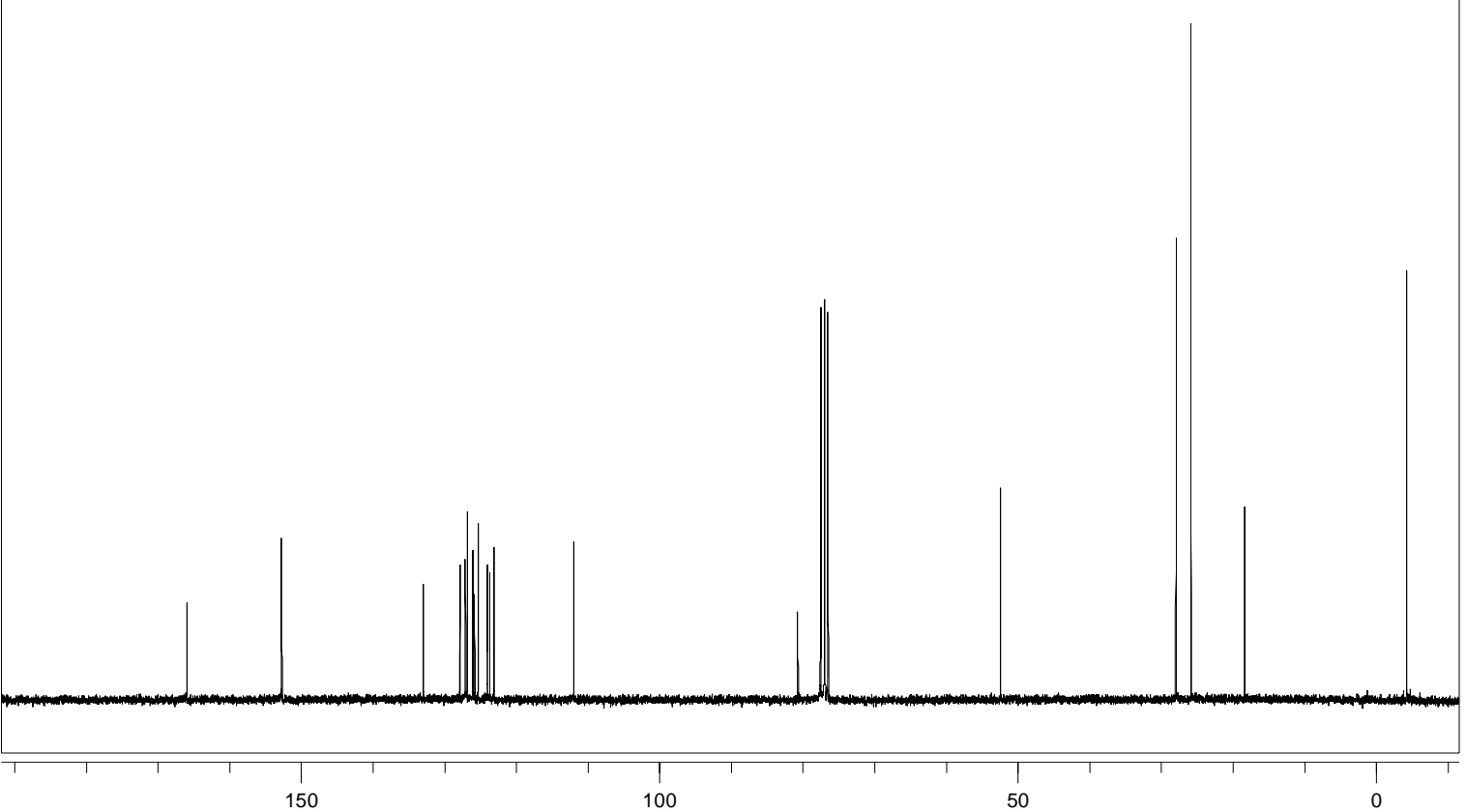

$\operatorname{ppm}(\mathrm{t} 1)$ 
NOESY spectrum of $\mathbf{1 3 .}$

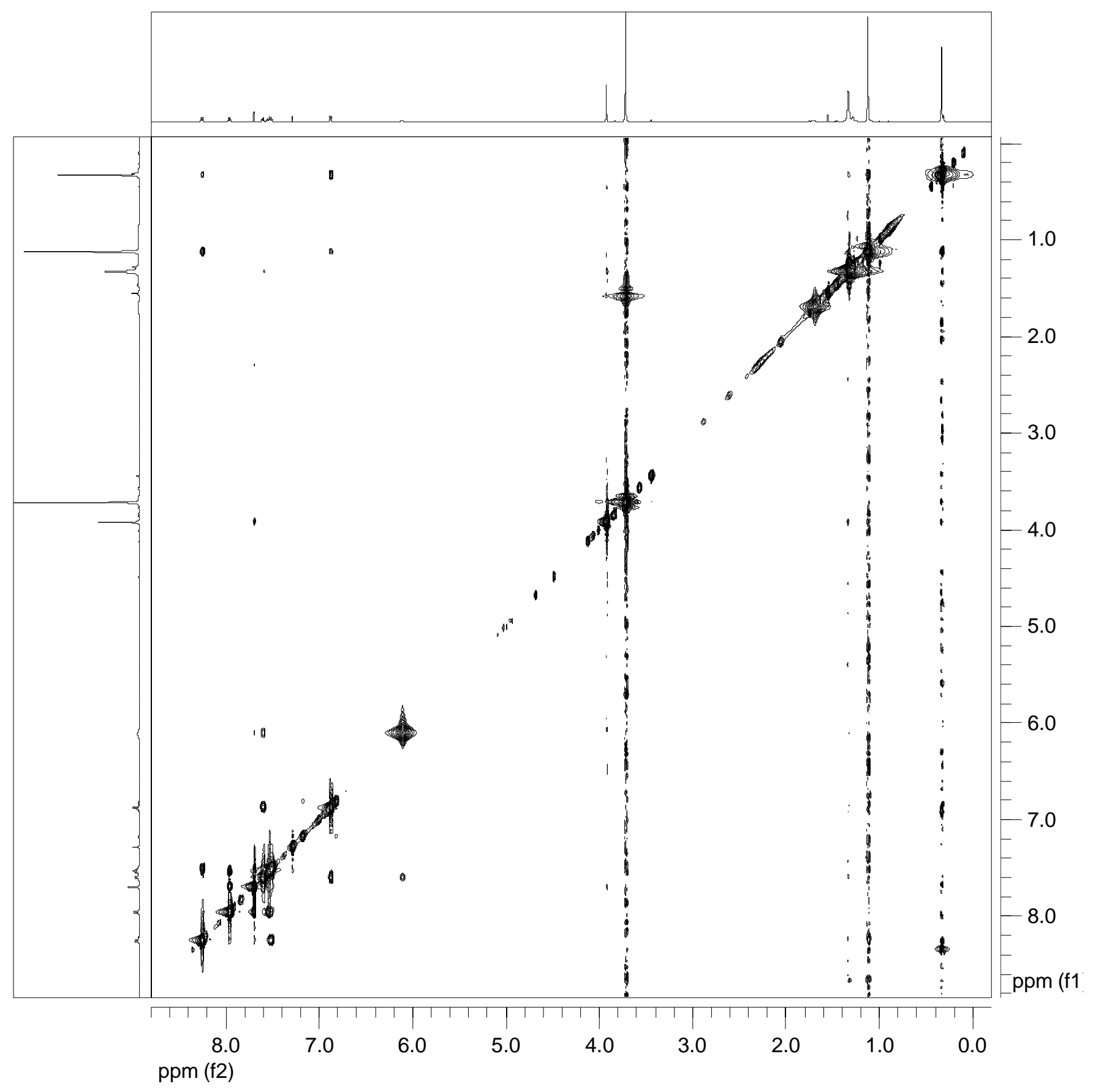


NOESY spectrum of $\mathbf{1 3}$ (zoom).

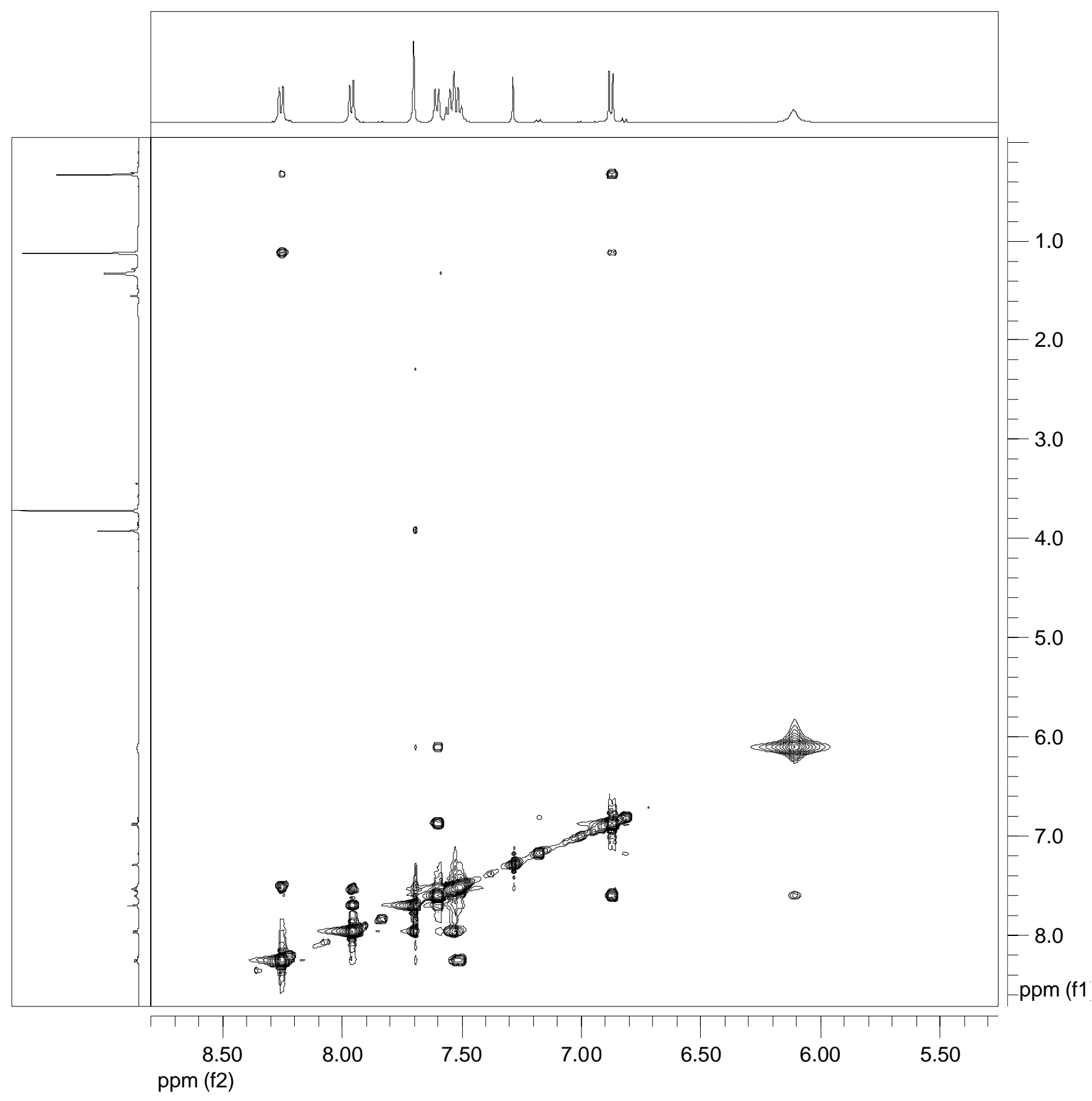


NMR spectrum of methyl 2-(tert-butyloxycarbonyl)amino-3-(1-(tert-butyldimethylsilyloxy) naphthalene-4-yl) acrylate (13). Mixture of isomers $(Z / E \sim 93 / 7)$.

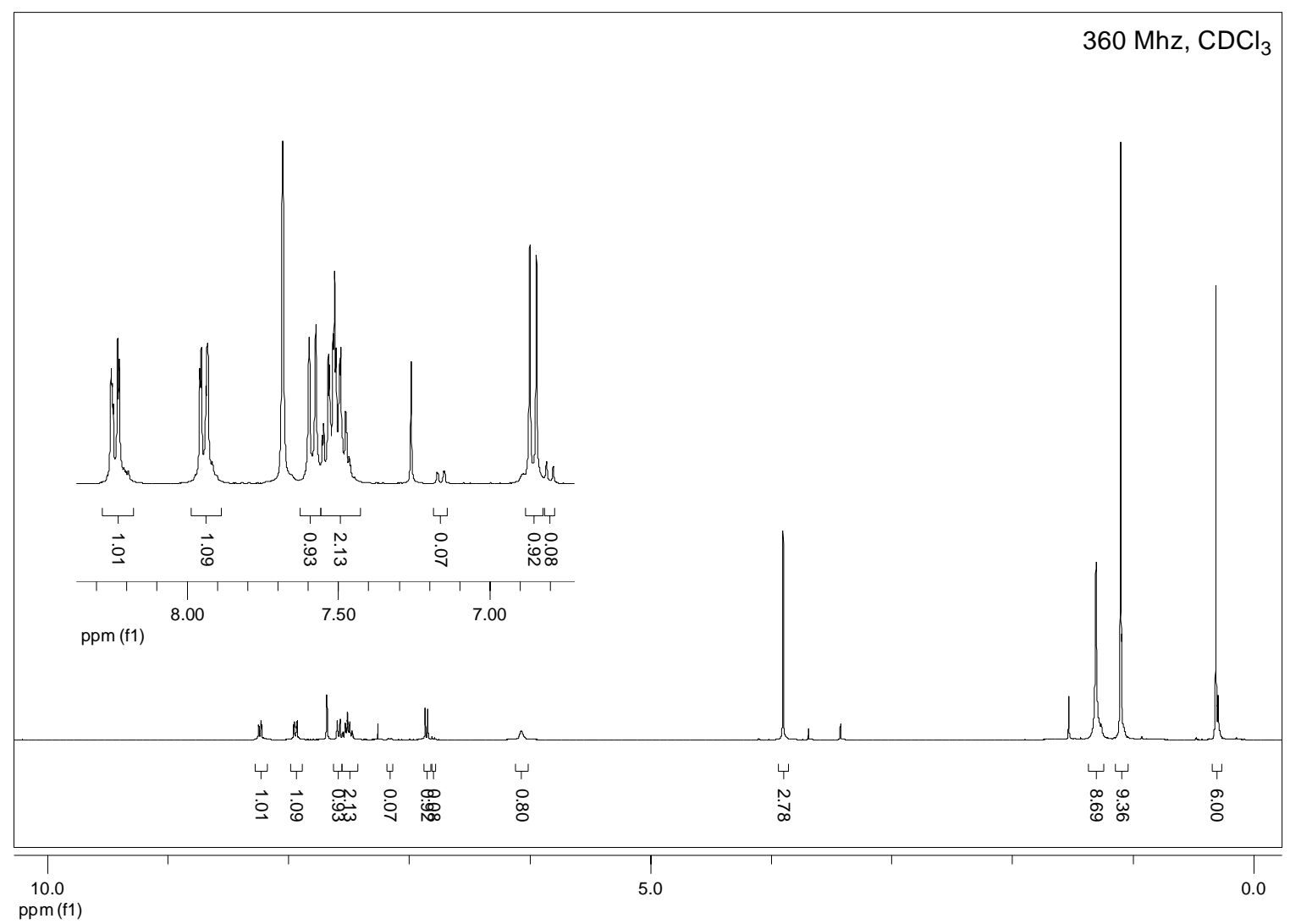


NMR spectra of (S)-N $N^{\alpha}$-tert-butyloxycarbonyl-1-(4-tert-butyldimethylsilyloxy)naphthylalanine methyl ester (14).
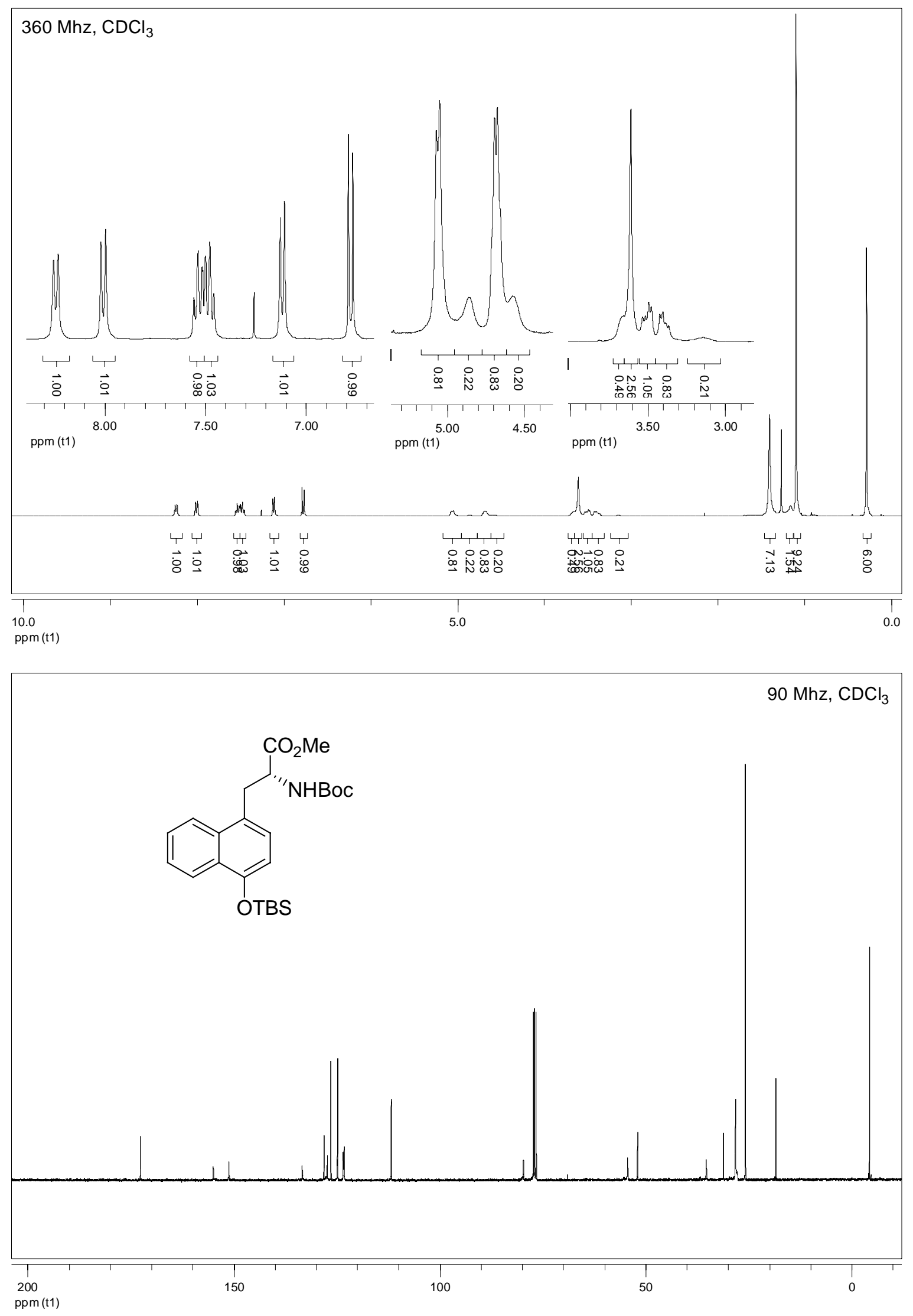
NMR spectra of (S)- $N^{\alpha}$-tert-butyloxycarbonyl-1-naphthylalanine (15).
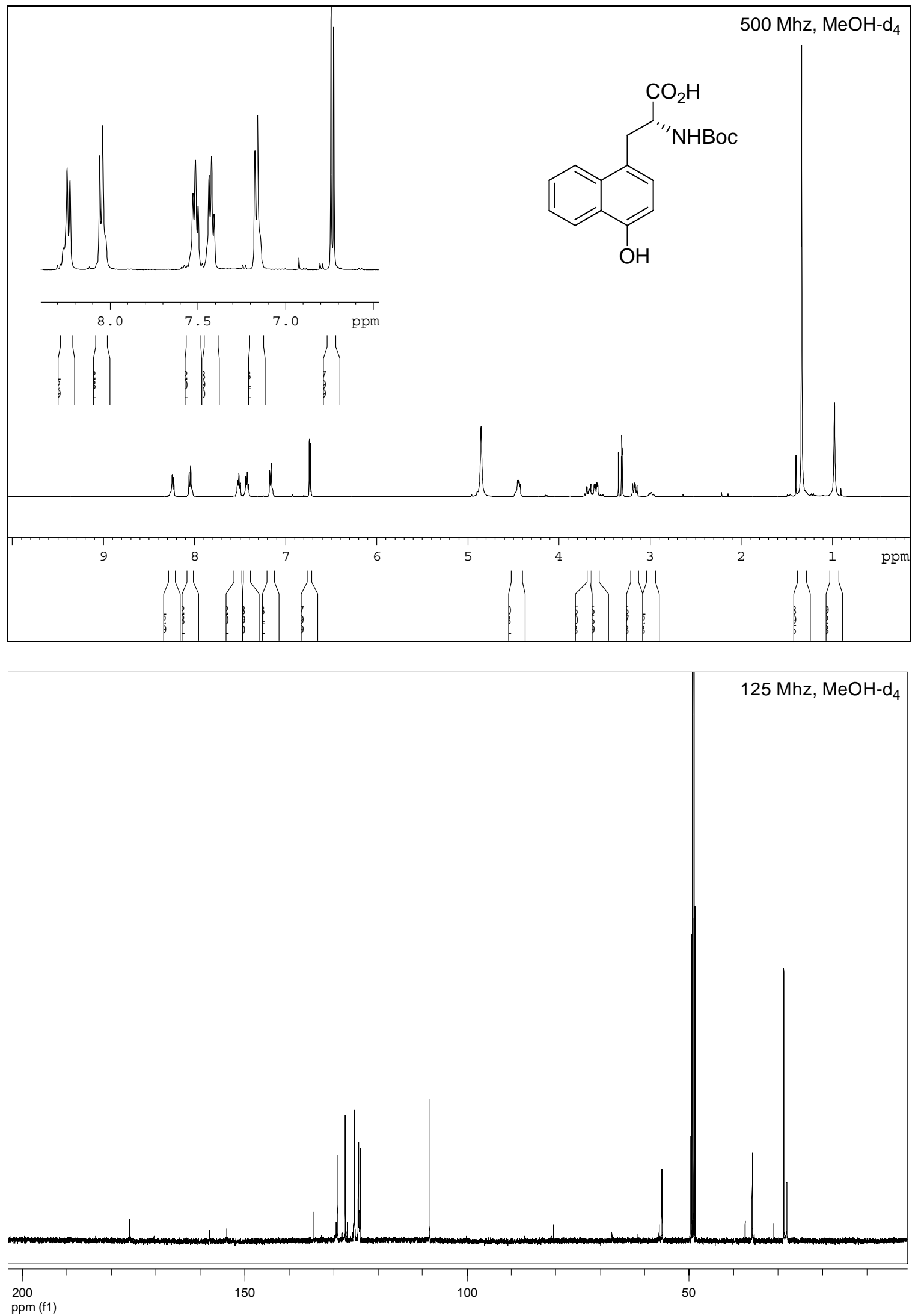
NMR spectra of (Z)-methyl 2-(benzyloxycarbonyl)amino-3-(1-(tert-butyldimethylsilyloxy) naphthalene-4-yl) acrylate (18).
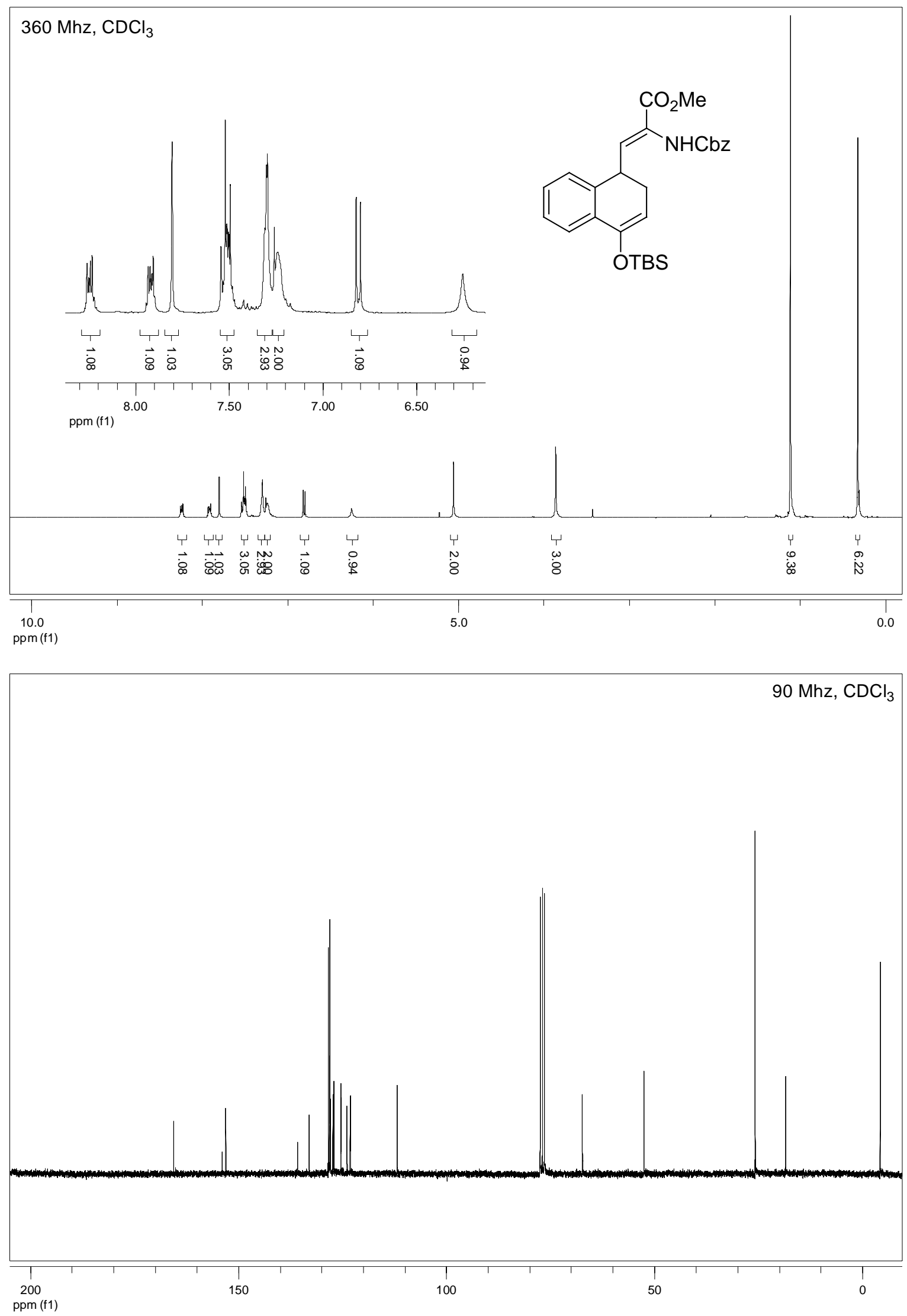
NOESY spectrum of $\mathbf{1 8 .}$

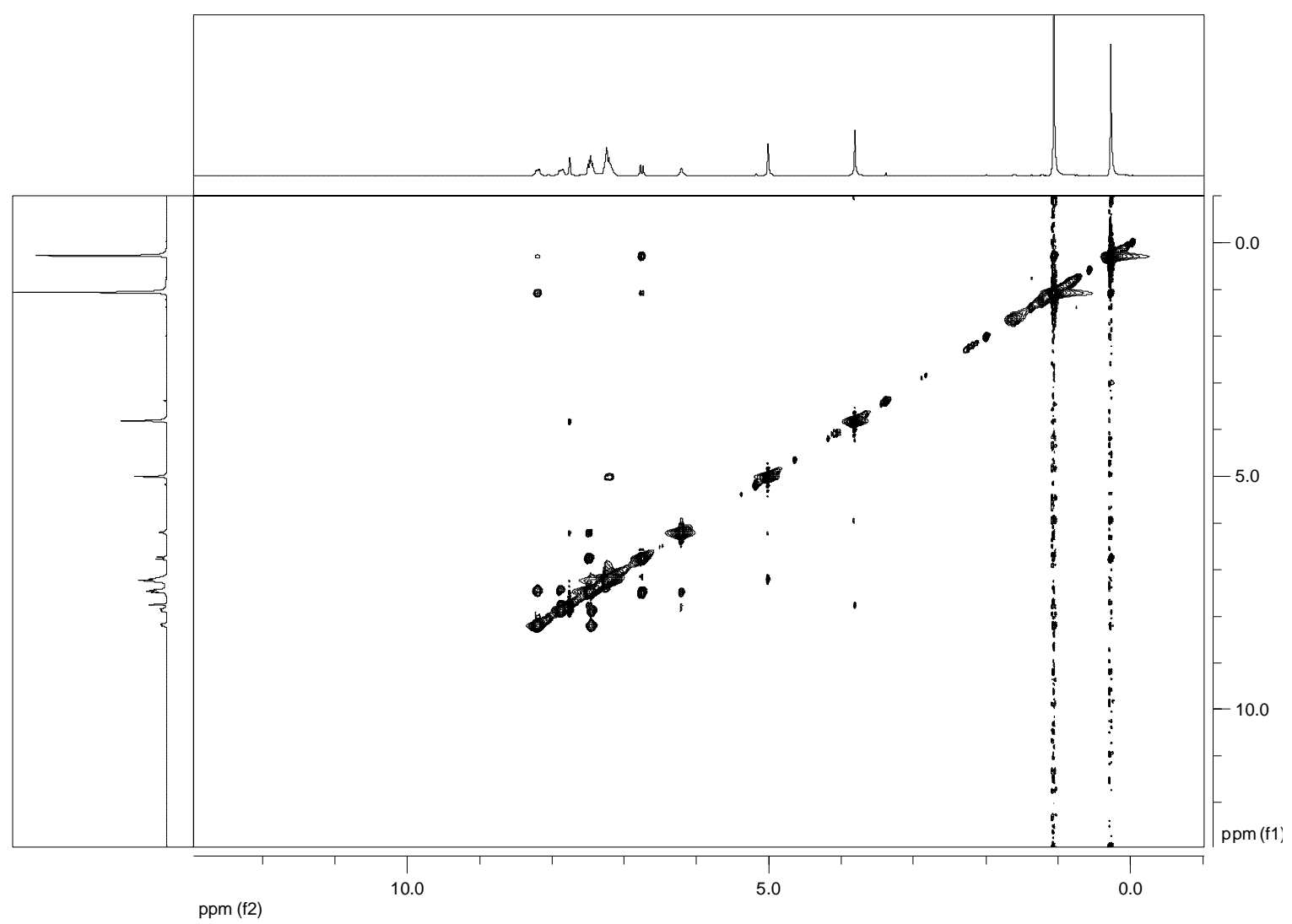

NOESY spectrum of $\mathbf{1 8}$ (zoom).

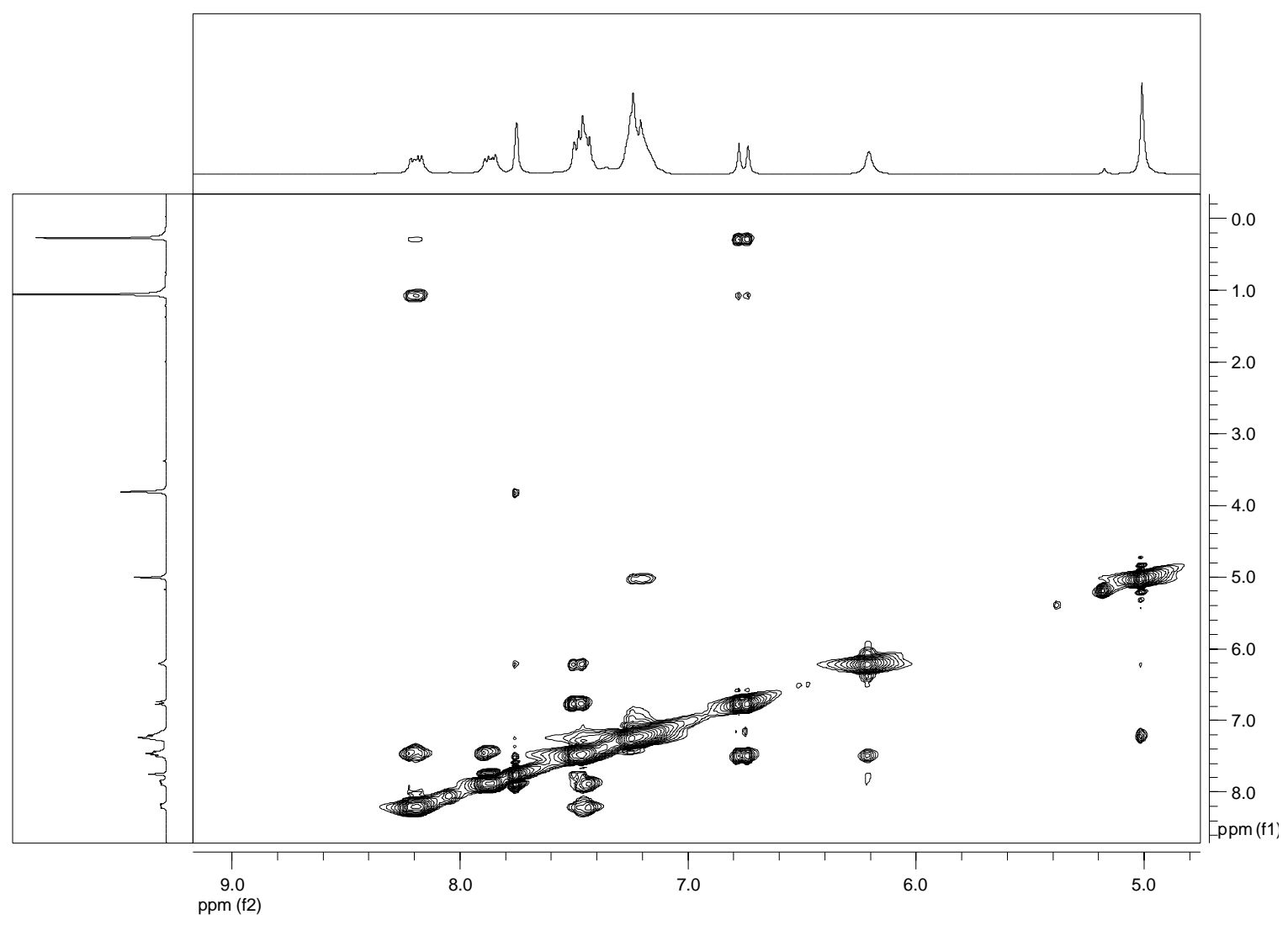


NMR spectra of $(R / S)-N^{\alpha}$-acetyl-1-(4-tert-butyldimethylsilyloxy)naphthylalanine methyl ester (19).

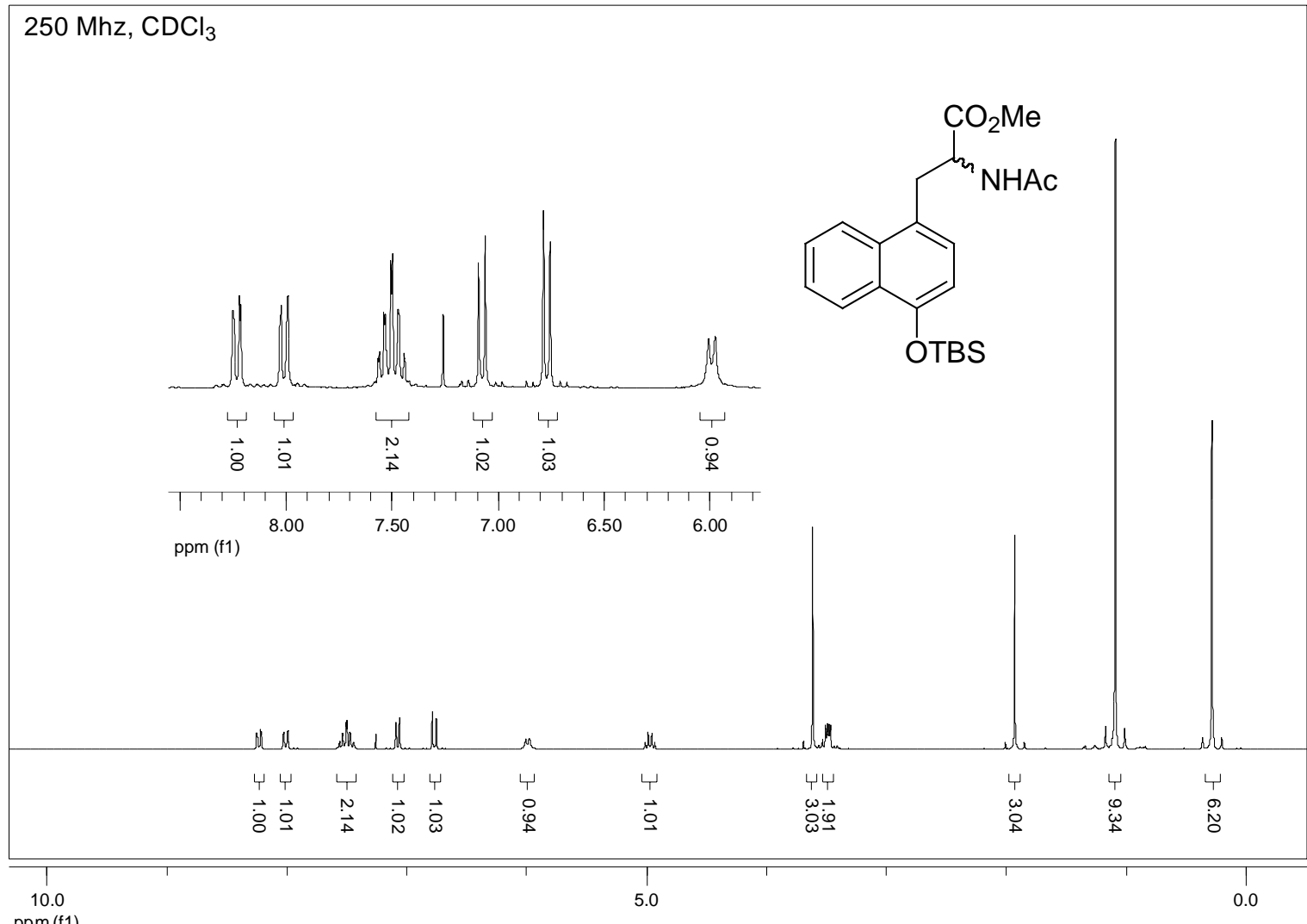

$63 \mathrm{Mhz}, \mathrm{CDCl}_{3}$

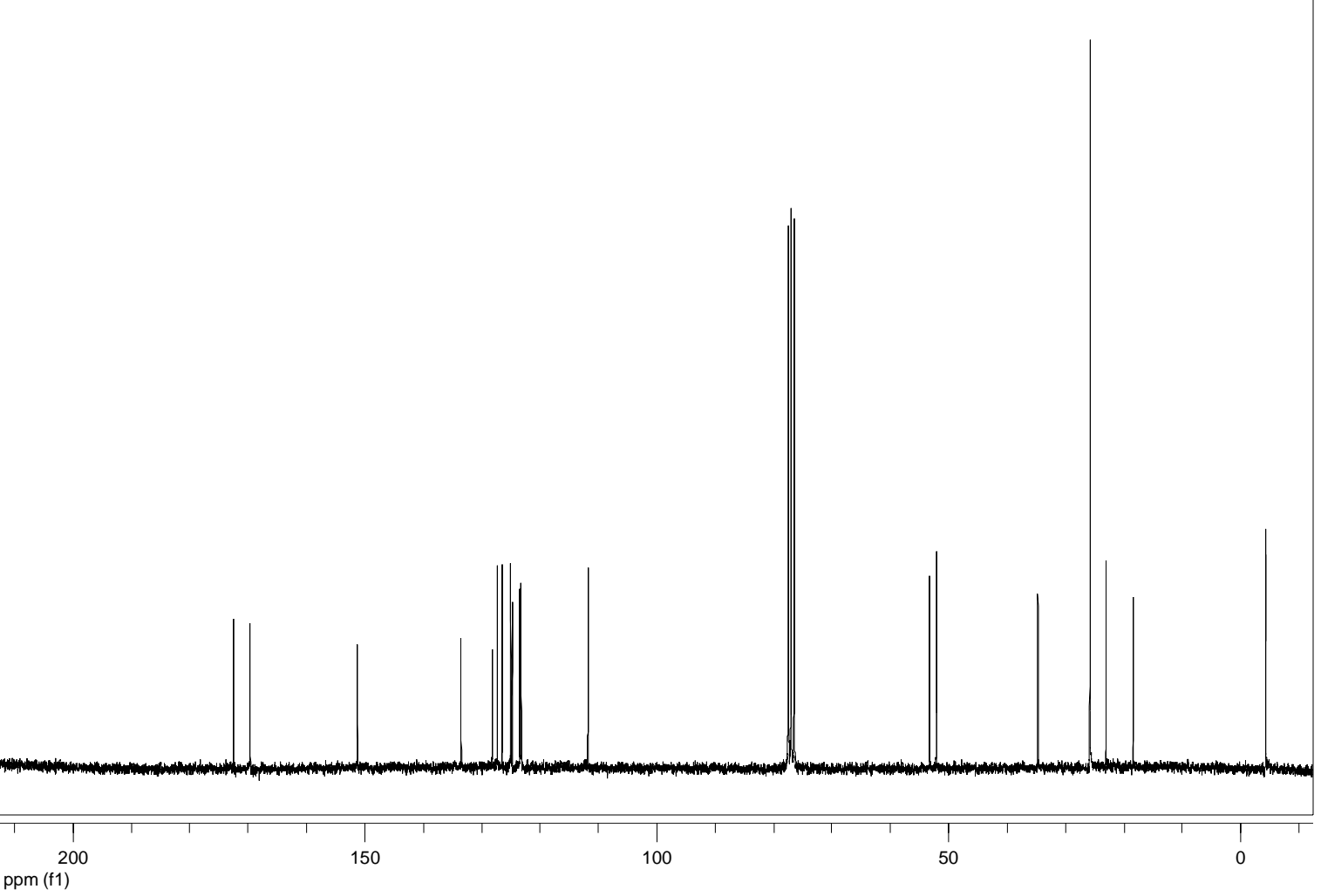


NMR spectra of $(R)-N^{\alpha}$-acetyl-1-(4-hydroxy)naphthylalanine (21).
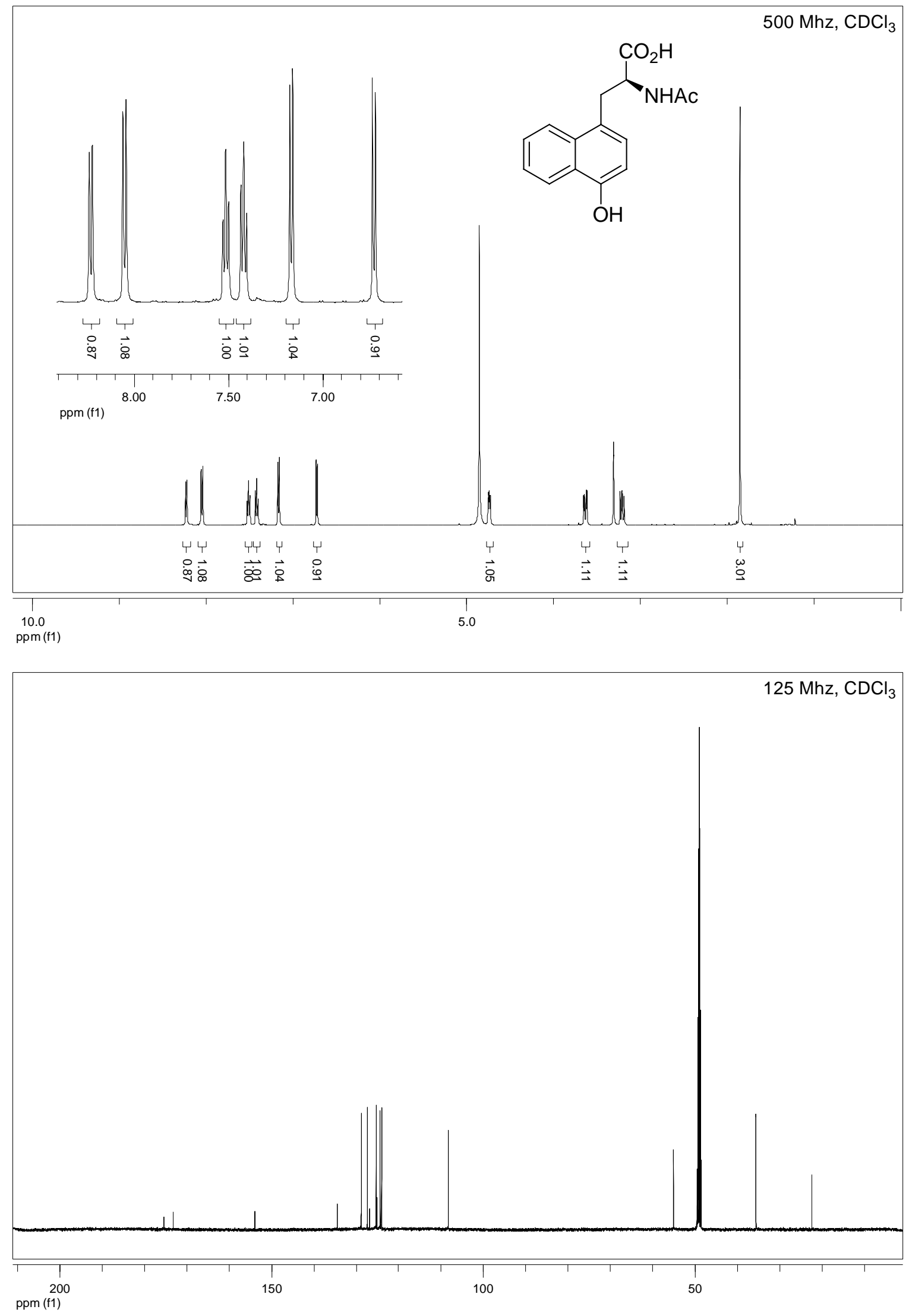
NMR spectra of (S)-1-(4-hydroxy)naphthylalanine methyl ester (22).

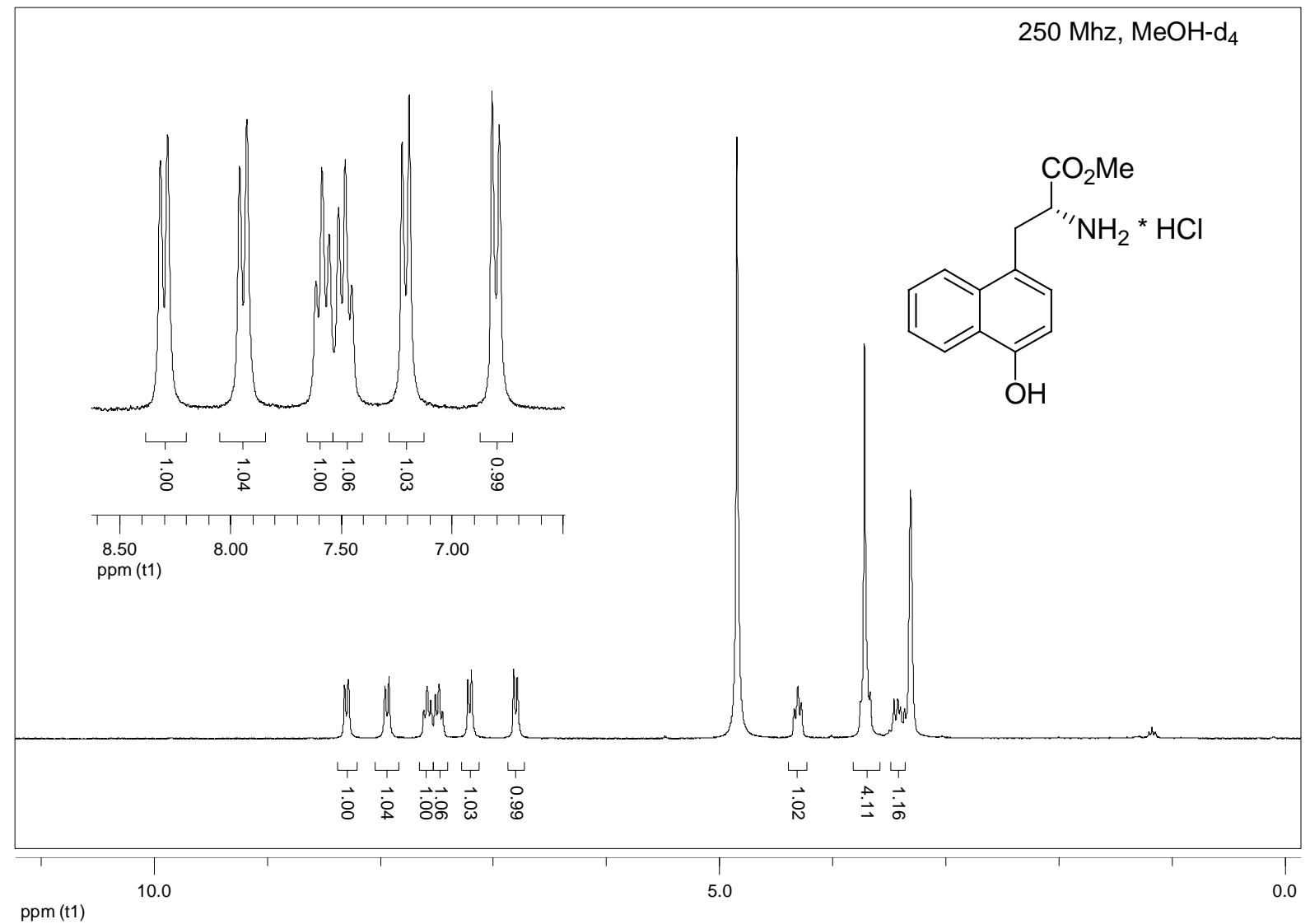

$226 \mathrm{Mhz}, \mathrm{MeOH}-\mathrm{d}_{4}$

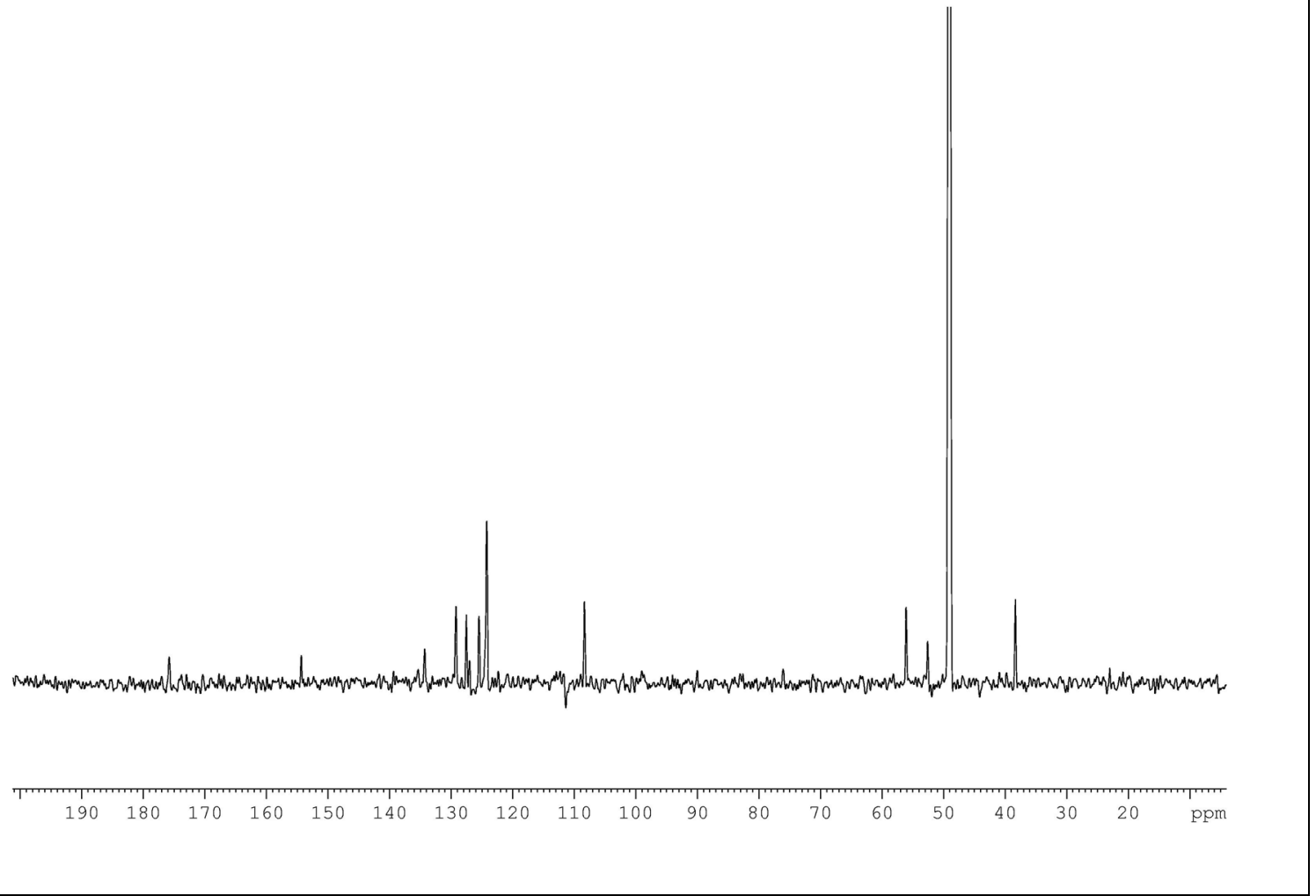


NMR spectra of H-m-(phenyl)Tyr-Val-OH (10a).
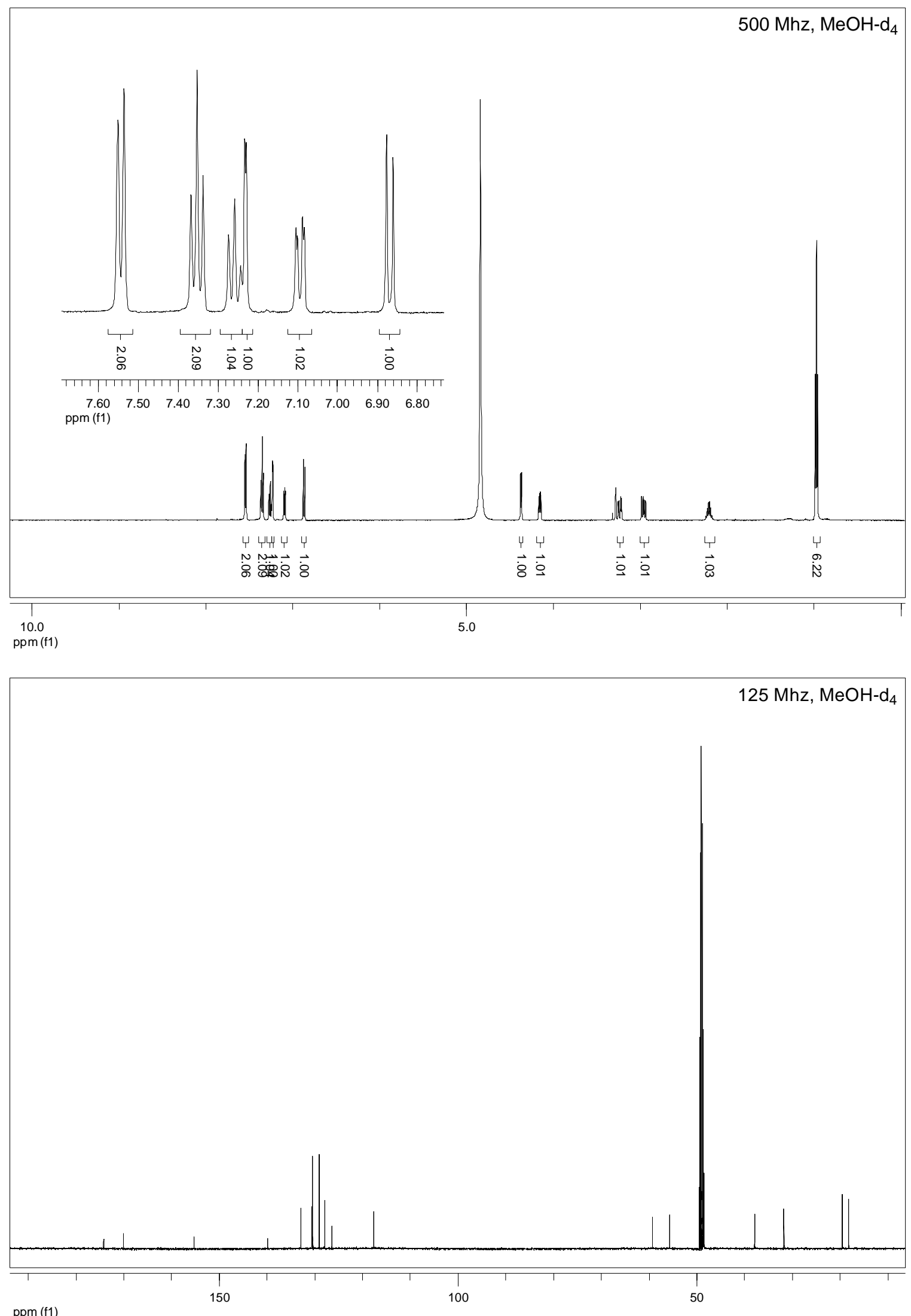
NMR spectra of H-m-(phenyl)Tyr-D-Val-OH (10c).
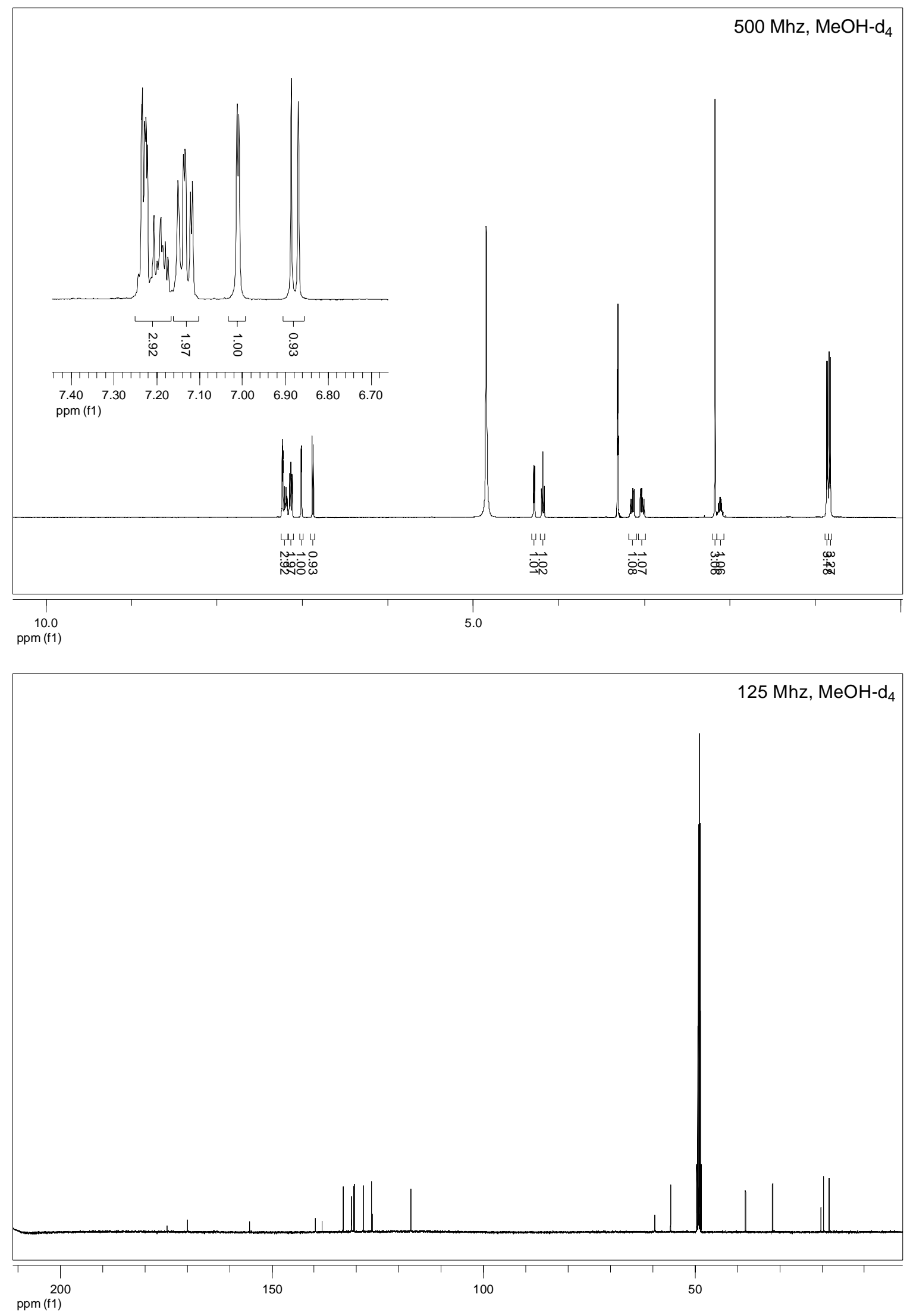
HPLC spectrum of 10a.

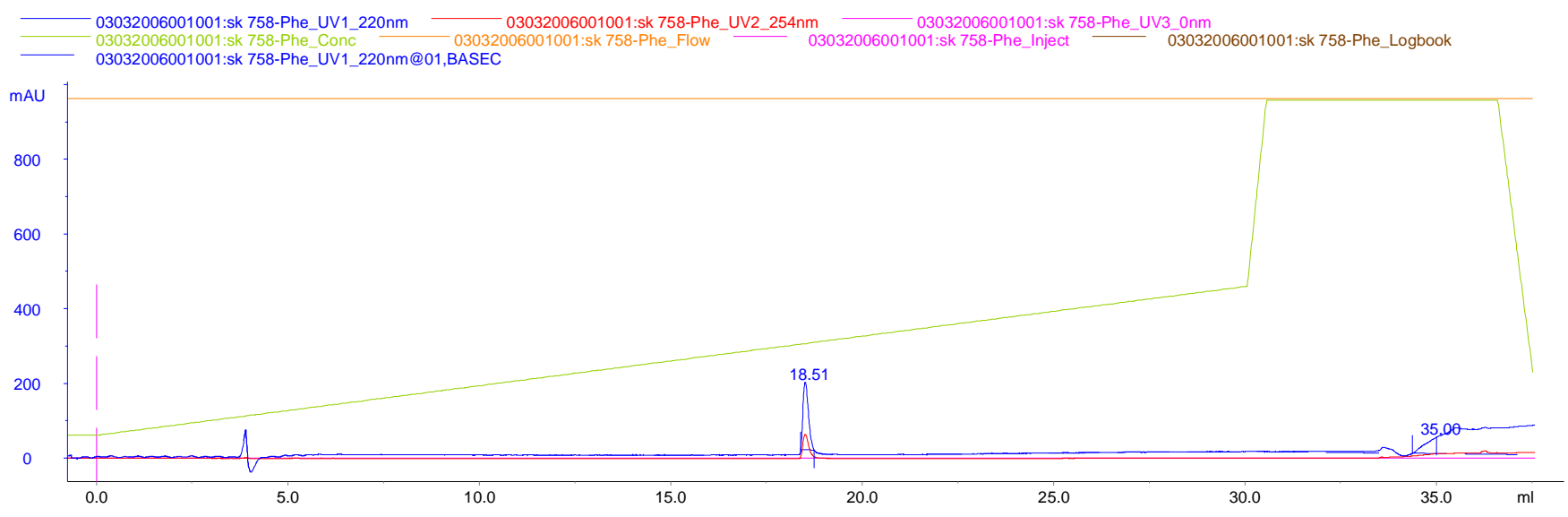

\section{HPLC spectrum of 10c.}

24022006001017:sk D-758-Phe_UV1_220nm 24022006001017:sk D-758-Phe_UV2_254nm - 24022006001017:sk D-758-Phe_UV3_0nm 24022006001017:Sk D-758-Phe_Conc 24022006001017:sk D-758-Phe_Flow _— 24022006001017:sk D-758-Phe_Inject — 24022006001017:sk D-758-Phe_Logbook 24022006001017:sk D-758-Phe_UV1_220nm@01,BASEC

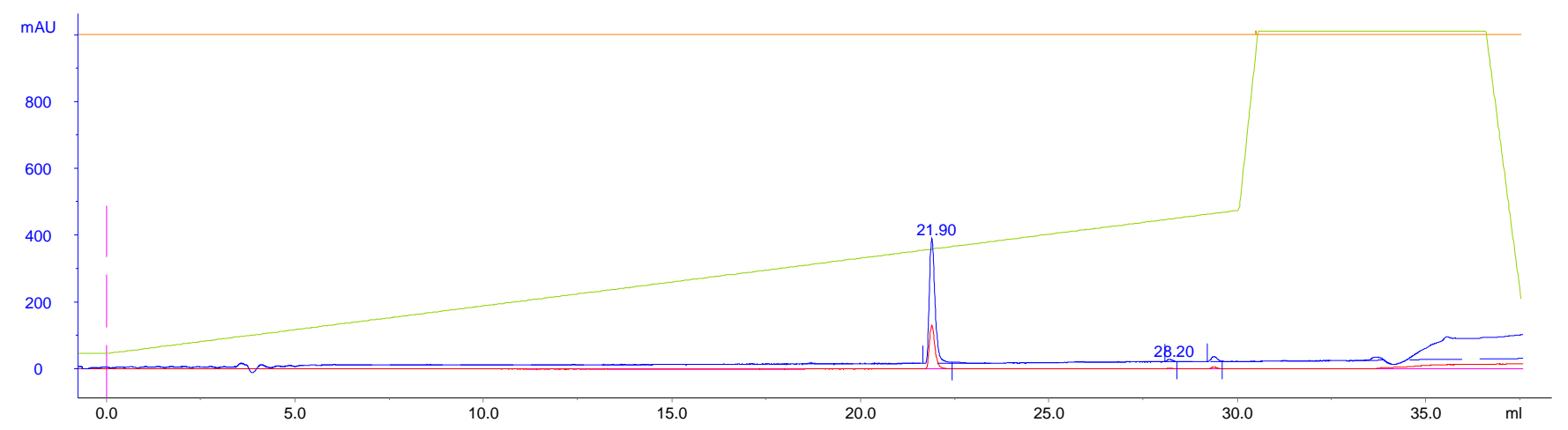


NMR spectra of H- $m$-(o-tolyl)Tyr-Val-OH (10b).

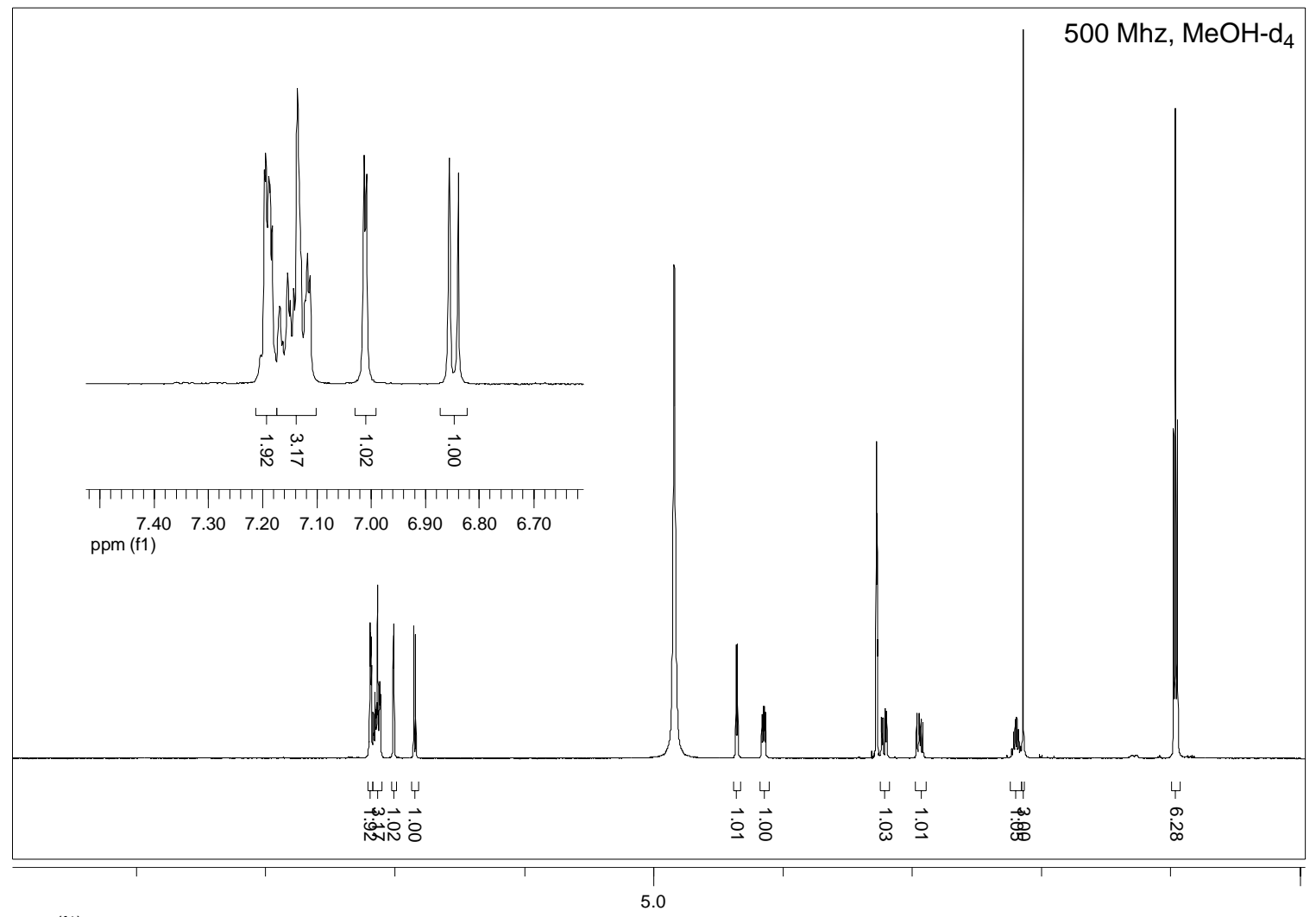

ppm (f1)

$125 \mathrm{Mhz}, \mathrm{MeOH}-\mathrm{d}_{4}$

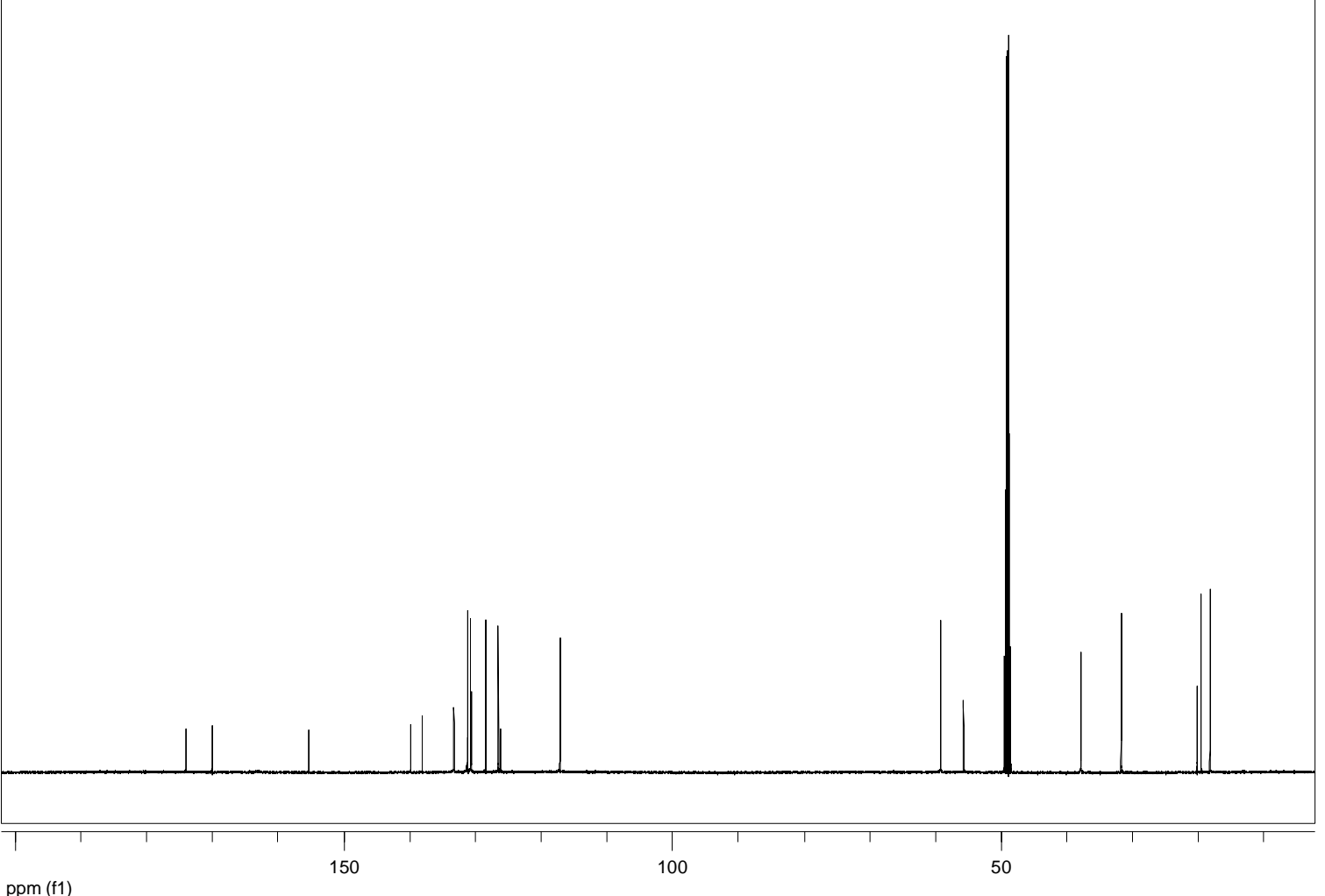

ppm (f1) 
NMR spectra of H-m-(o-tolyl)Tyr-D-Val-OH (10d).
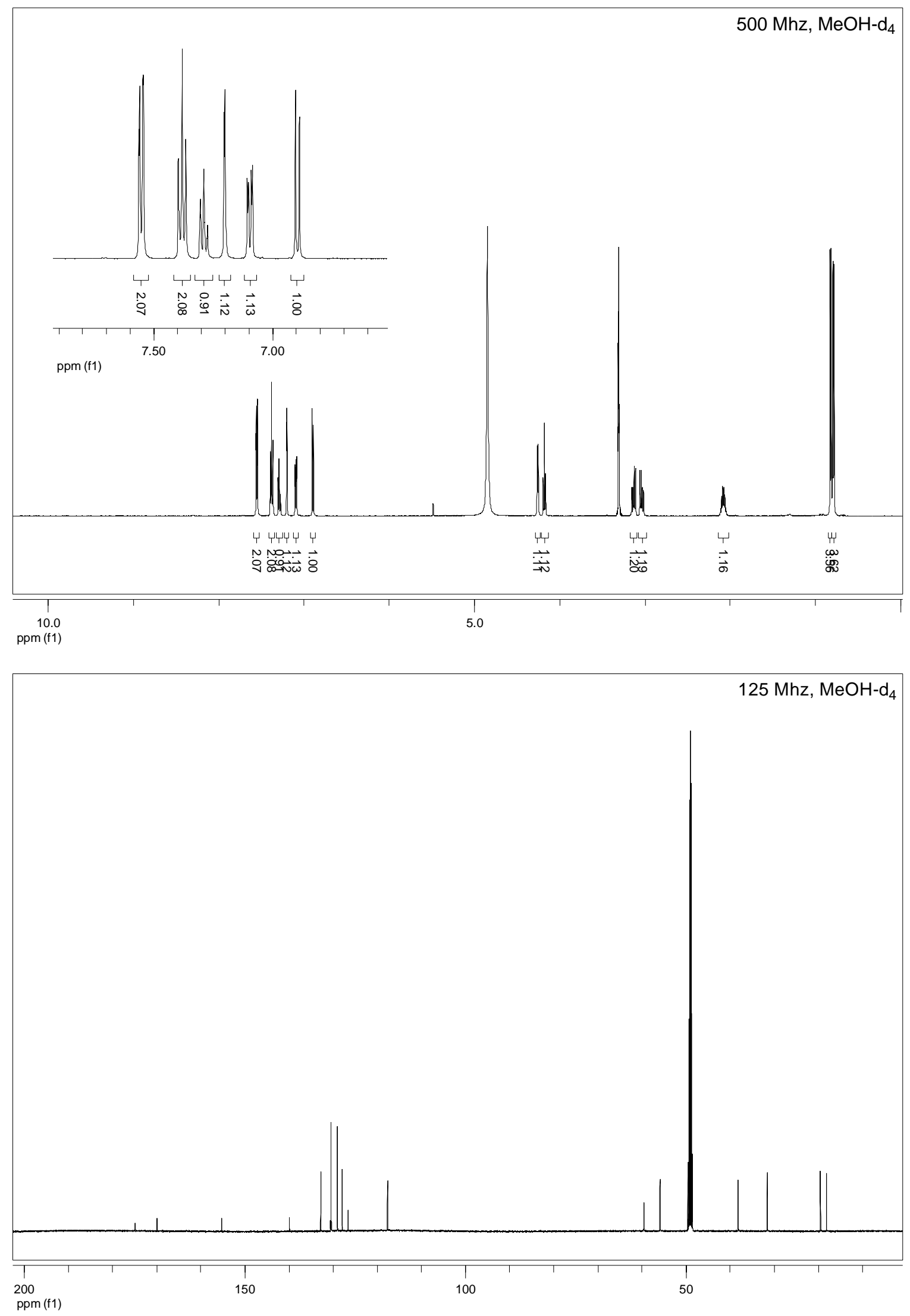
HPLC spectrum of $\mathbf{1 0 b}$.

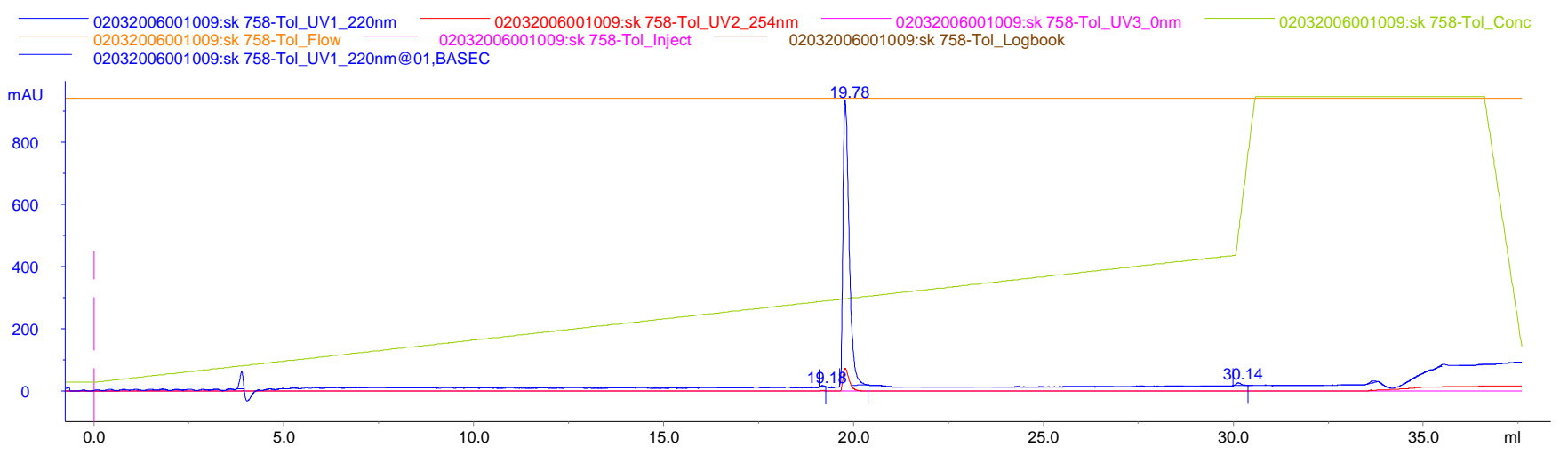

\section{HPLC spectrum of 10d.}

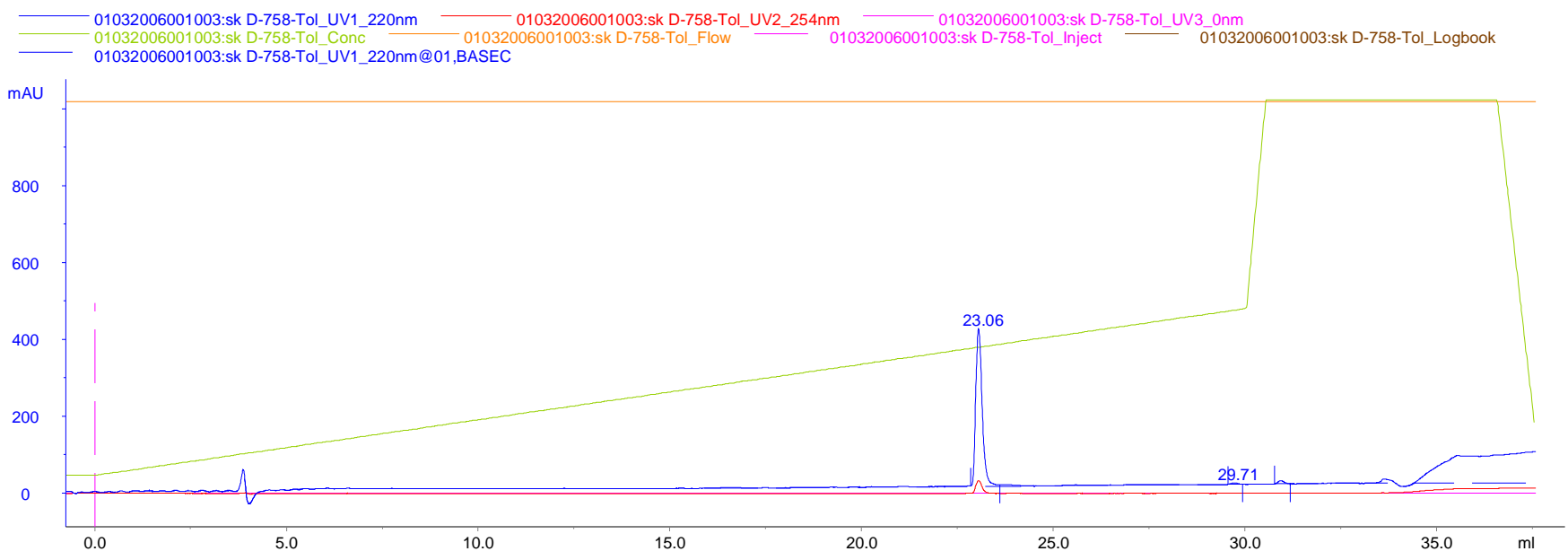


NMR spectra of H-(4-hydroxy)Nal-Val-OH (16).
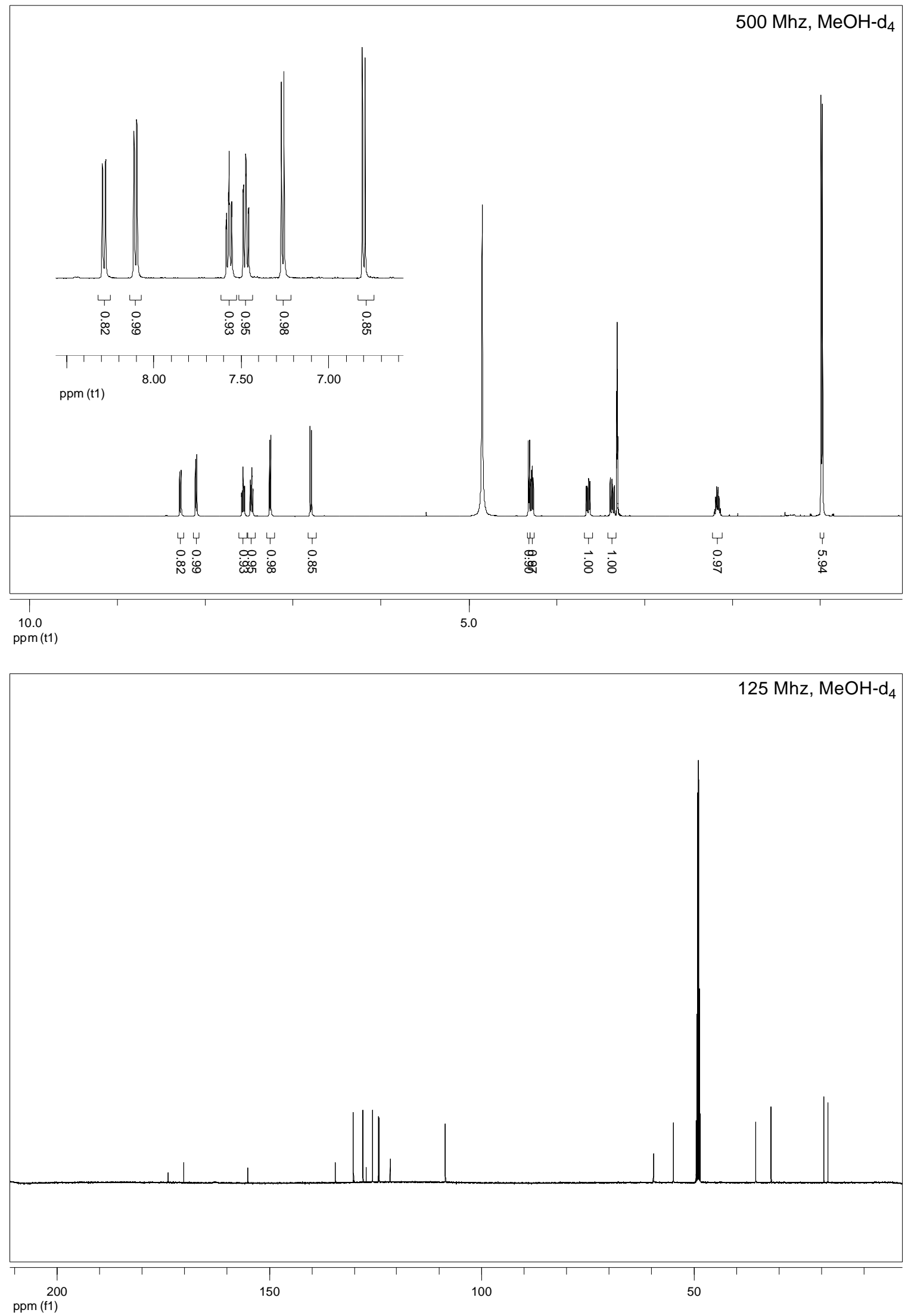
${ }^{1} \mathrm{H}-\mathrm{NMR}$ spectrum of H-(D/L)-1-(4-hydroxy)Nal-Val-OH (17).

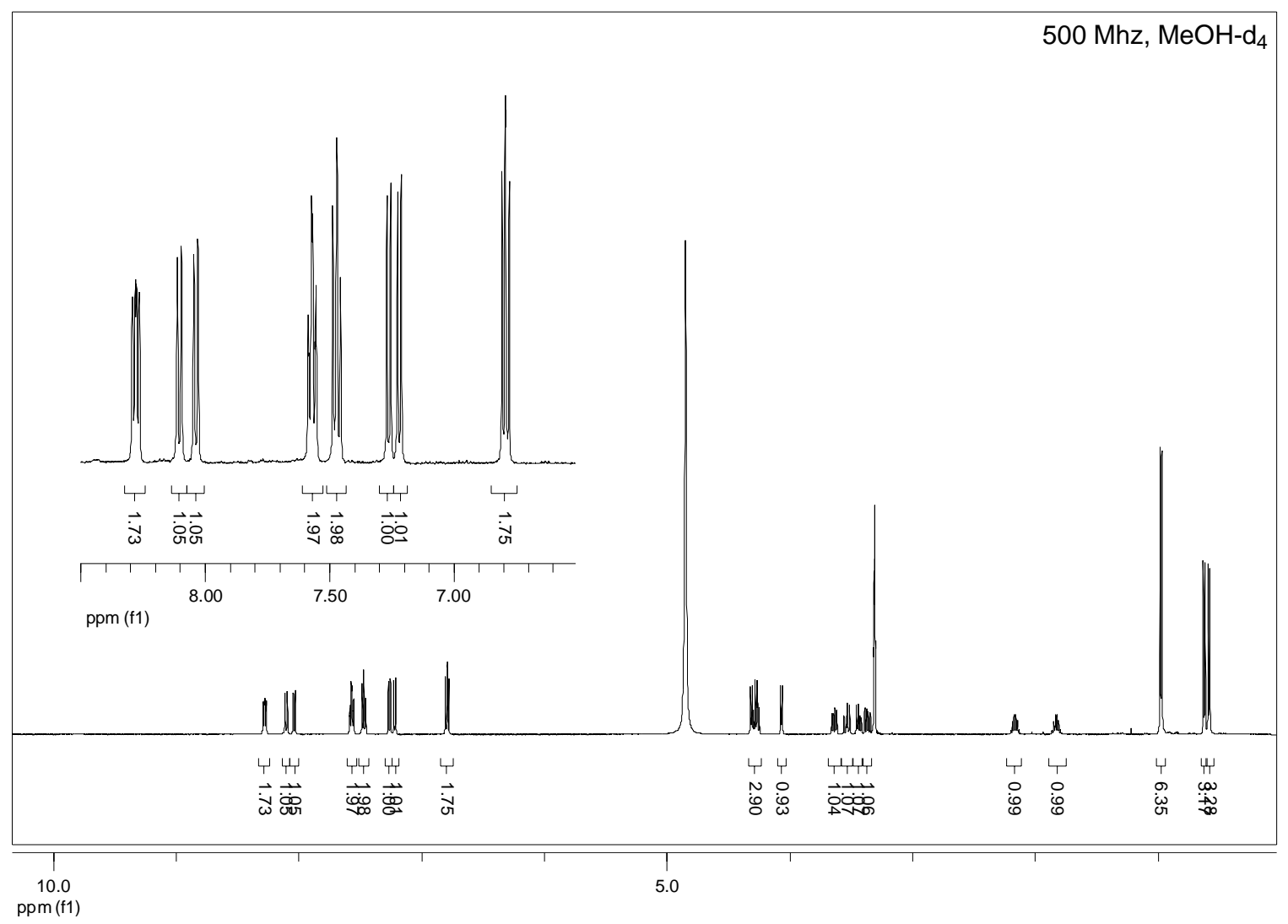




\section{HPLC spectrum of $\mathbf{1 6 .}$}

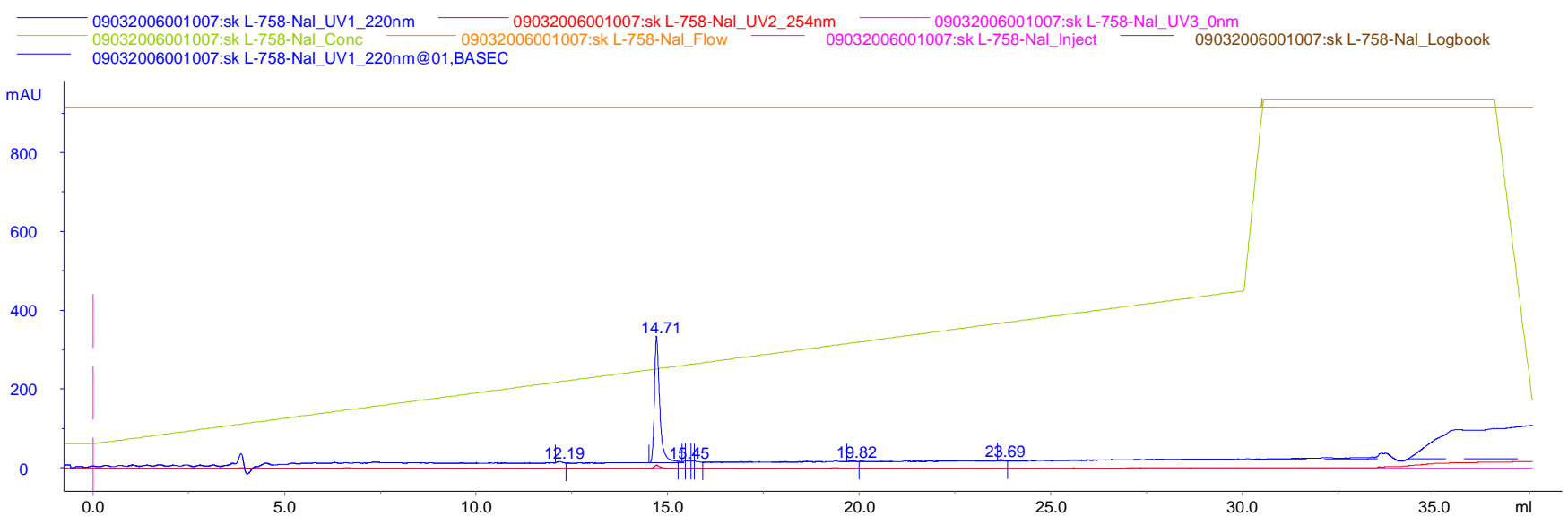

\section{HPLC spectrum of 17.}

03032006001004:sk rac.-758-Nal_UV1_220nm - 03032006001004:sk rac.-758-Nal_UV2_254nm 03032006001004:sk rac.-758-Nal_Conc 03032006001004:sk rac.-758-Nal_Flow _ —— 03032006001004:sk rac.-758-Nal_Inject — 03032006001004:sk rac.-758-Nal_Logbook

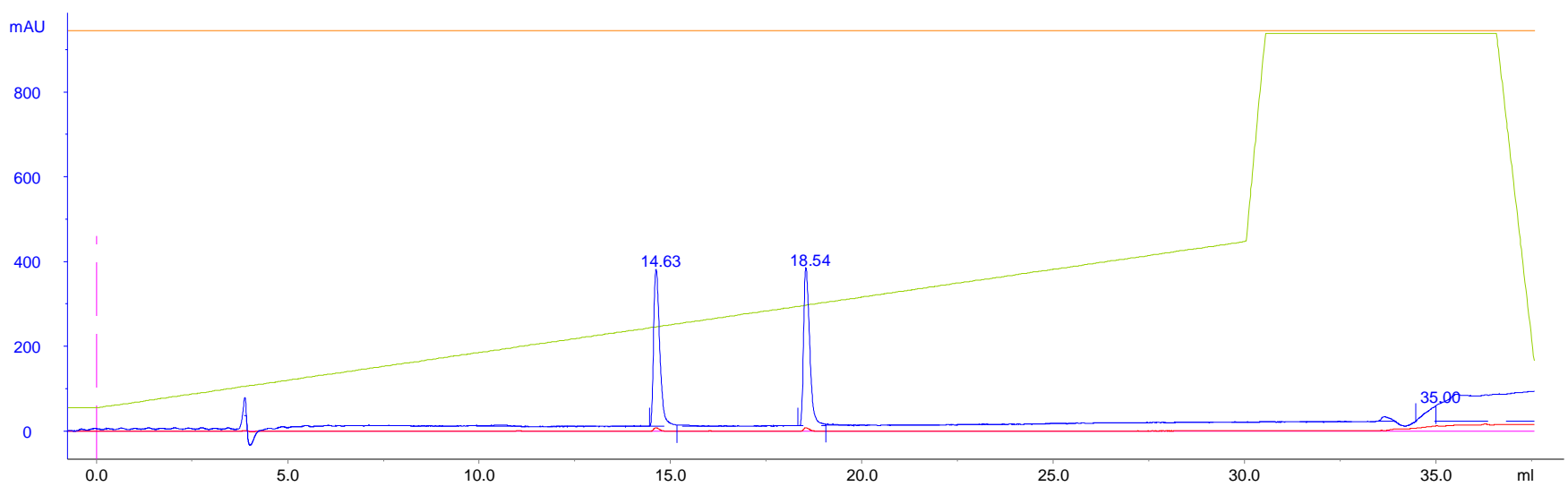

\section{HPLC spectrum of $\mathbf{1 6}$ (zoom).}

09032006001007:sk L-758-Nal_UV1_220nm - 09032006001007:sk L-758-Nal_UV2_254nm - 09032006001007:sk L-758-Nal_UV3_0nm

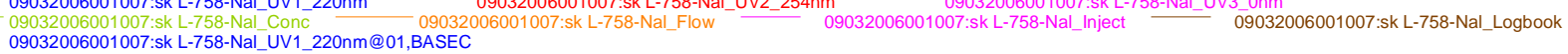

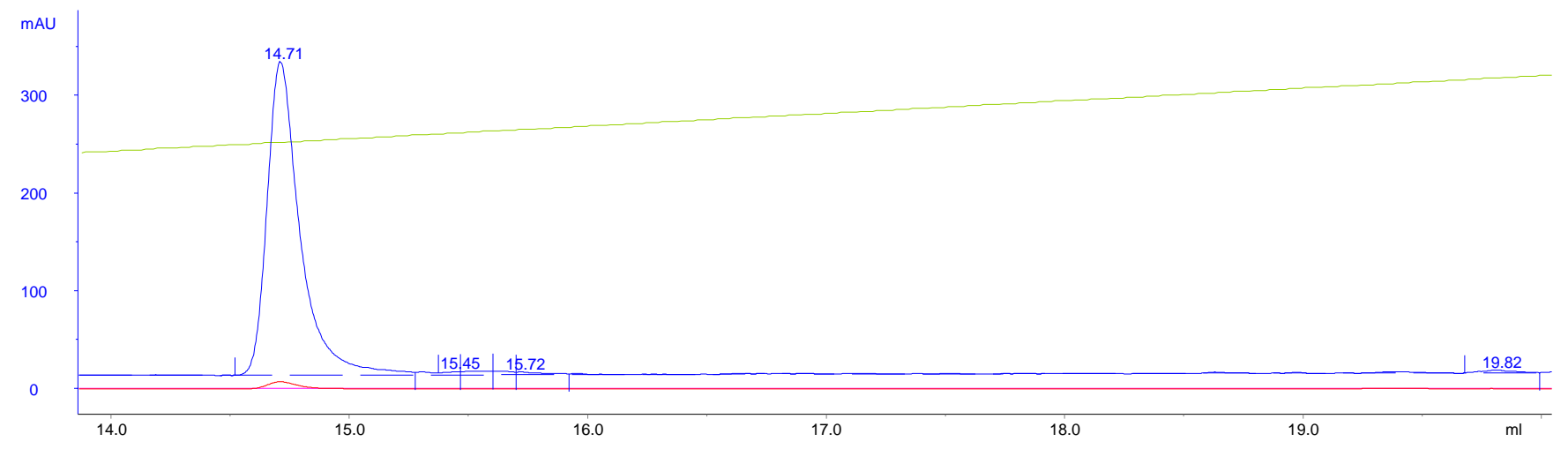




\section{References}

1. Walker, W. H.; Rokita, S. E., J. Org. Chem. 2003, 68, 1563-1566.

2. Fields, G. B.; Noble, R. L., Int. J. Pept. Res. 1990, 35, 161-214. 